\title{
Basics of Bose-Einstein Condensation
}

\author{
V.I. Yukalov \\ Bogolubov Laboratory of Theoretical Physics, \\ Joint Institute for Nuclear Research, Dubna, Russia \\ and \\ National Institute of Optics and Photonics, \\ University of São Paulo, São Carlos, Brazil
}

\begin{abstract}
The review is devoted to the elucidation of the basic problems arising in the theoretical investigation of systems with Bose-Einstein condensate. Understanding these challenging problems is necessary for the correct description of Bose-condensed systems. The principal problems considered in the review are as follows: (i) What is the relation between Bose-Einstein condensation and global gauge symmetry breaking? (ii) How to resolve the Hohenberg-Martin dilemma of conserving versus gapless theories? (iii) How to describe Bose-condensed systems in strong spatially random potentials? (iv) Whether thermodynamically anomalous fluctuations in Bose systems are admissible? (v) How to create nonground-state condensates? Detailed answers to these questions are given in the review. As examples of nonequilibrium condensates, three cases are described: coherent modes, turbulent superfluids, and heterophase fluids.
\end{abstract}

PACS: 03.75.Hh, 03.75.Kk, 03.75.Nt, 05.30.Ch, 05.30.Jp, 67.85.Bc, 67.85.De, 67.85.Jk 


\section{Contents}

\section{Principal Theoretical Problems}

2. Criteria of Bose-Einstein Condensation

2.1 Einstein Criterion

2.2 Yang Criterion

2.3 Penrose-Onsager Criterion

2.4 Order Indices

2.5 Condensate Existence

\section{Gauge Symmetry Breaking}

3.1 Gauge Symmetry

3.2 Symmetry Breaking

3.3 Ginibre Theorem

3.4 Bogolubov Theorem

3.5 Roepstorff Theorem

\section{General Self-Consistent Approach}

4.1 Representative Ensembles

4.2 Bogolubov Shift

4.3 Grand Hamiltonian

4.4 Variational Principle

4.5 Equations of Motion

\section{Superfluidity in Quantum Systems}

5.1 Superfluid Fraction

5.2 Moment of Inertia

5.3 Equivalence of Definitions

5.4 Local Superfluidity

5.5 Superfluidity and Condensation

\section{Equilibrium Uniform Matter}

6.1 Information Functional

6.2 Momentum Representation

6.3 Condensate Fraction

6.4 Green Functions

6.5 Hugenholtz-Pines Relation

\section{Hartree-Fock-Bogolubov Approximation}

7.1 Nonuniform Matter

7.2 Bogolubov Transformations

7.3 Uniform Matter

7.4 Local-Density Approximation

7.5 Particle Densities 


\section{Local Interaction Potential}

8.1 Grand Hamiltonian

8.2 Evolution Equations

8.3 Equilibrium Systems

8.4 Uniform Systems

8.5 Atomic Fractions

\section{Disordered Bose Systems}

9.1 Random Potentials

9.2 Stochastic Decoupling

9.3 Perturbation-Theory Failure

9.4 Local Correlations

9.5 Bose Glass

10. Particle Fluctuations and Stability

10.1 Stability Conditions

10.2 Fluctuation Theorem

10.3 Ideal-Gas Instability

10.4 Trapped Atoms

10.5 Interacting Systems

\section{Nonground-State Condensates}

11.1 Coherent Modes

11.2 Trap Modulation

11.3 Interaction Modulation

11.4 Turbulent Superfluid

11.5 Heterophase Fluid

\section{Conclusions}




\section{Principal Theoretical Problems}

In recent years, the topic of Bose-Einstein condensation has been attracting very high attention. There have been published the books $[1,2]$ and a number of review articles (e.g. [3-12]). This great attention is mainly due to a series of beautiful experiments with trapped atoms, accomplished in many laboratories of different countries and promising a variety of interesting applications. The interpretation of experiments requires the development of theory. It is well known that there is nothing more practical than a good theory. Only a correct theory allows for the proper understanding of experiments and can suggest appropriate and realistic technical applications.

The theory of real systems with Bose-Einstein condensate was advanced by Bogolubov [13-16] who considered uniform weakly nonideal low-temperature Bose gas. Extensions to nonuniform zero-temperature weakly interacting gas are due to Gross [17-19], Ginzburg and Pitaevskii [20], and Pitaevskii [21]. This approach has been the main tool for describing Bose-condensed systems, since the majority of initial experiments with trapped atoms had been accomplished with weakly interacting Bose gases at low temperatures, using the techniques of cooling and trapping [22].

Since London [23], it is assumed that superfluidity in ${ }^{4} \mathrm{He}$ is accompanied by Bose-Einstein condensation, although detecting the condensate fraction in helium is a rather difficult experimental task. The existence in liquid helium of Bose-Einstein condensate with zero momentum has not been directly proved, without model assumptions, though the majority of experiments are in agreement with the existence of condensate fraction of about $10 \%$ [24]. The possibility that superfluidity is accompanied by mid-range atomic correlations [25-27] or that it is due to the appearance in superfluid helium of a condensate with a finite modulus of momentum [28-31] have also been discussed. In his works on superfluid helium, Landau [32] has never assumed the condensate existence. This is why the direct observation of Bose-Einstein condensation of trapped atoms has become so important and intensively studied phenomenon [1-12].

The trapped Bose gases are dilute and can be cooled down to very low temperatures. Usually, they also are weakly interacting. Thus, cold trapped atomic gases have become the ideal object for the application of the Bogolubov theory [13-16].

However, by employing the Feshbach resonance techniques [33,34] it is possible to vary atomic interactions, making them arbitrarily strong. In addition, the properties of trapped gases at nonzero temperature have also to be properly described. But the Bogolubov approximation, designed for weakly interacting low-temperature systems, cannot be applied for Bose systems at finite interactions and temperature.

Attempts to use the Hartree-Fock-Bogolubov approximation resulted in the appearance of an unphysical gap in the spectrum $[35,36]$. While there should be no gap according to the Hugenholtz-Pines [37] and Bogolubov [16] theorems. This gap cannot be removed without loosing the self-consistency of theory, which would lead to the distortion of conservation laws and thermodynamic relations [16]. The situation was carefully analyzed by Hohenberg and Martin [38], who showed that, as soon as the global gauge symmetry, associated with the Bose-Einstein condensation, is broken, any theory, in the frame of the grand canonical ensemble, becomes either nonconserving or acquires a gap in the spectrum. This dramatic conclusion is known as the Hohenberg-Martin dilemma of conserving versus gapless theories. In this review, it is shown how a correct self-consistent theory has to be developed, being both conserving and gapless, and being valid for finite temperatures and arbitrary interactions.

In the Bogolubov approach, the global gauge symmetry $U(1)$ is broken, which yields BoseEinstein condensation. Hence, this gauge symmetry breaking is a sufficient condition for condensation. But maybe it is not necessary? Some researchers state that Bose-Einstein condensation 
does not require any symmetry breaking. This delusion is explained in the review, where it is emphasized that the gauge symmetry breaking is the necessary and sufficient condition for Bose-Einstein condensation.

In recent literature on Bose systems, there often happens a very unfortunate mistake, when one omits anomalous averages, arising because of the gauge symmetry breaking. But it is straightforward to show that this omission is principally wrong from the precise mathematical point of view. To get an excuse for the unjustified omission of anomalous averages, one ascribes such an omission to Popov, terming this trick "Popov approximation". Popov, however, has never suggested such an incorrect trick, which can be easily inferred from his original works $[39,40]$.

The general self-consistent theory, presented in the review, is based on the Bogolubov shift of field operators, which explicitly breaks the gauge symmetry. The theory is valid for arbitrary interacting Bose systems, whether equilibrium or nonequilibrium, uniform or nonuniform, in the presence of any external potentials, and at any temperature. External potentials of a special type are spatially random potentials. For treating the latter, one often uses perturbation theory with respect to disorder. However, it is possible to show that such perturbation theory can be misleading, yielding wrong results. In this review, a method is described that can be used for disorder potentials of any strength.

One of the most confusing problems, widely discussed in recent literature, is the occurrence of thermodynamically anomalous particle fluctuations in Bose-condensed systems. In the review, a detailed explanation is given that such anomalous fluctuations cannot arise in any real system, since their presence would make the system unstable, thus, precluding its very existence. The appearance of such anomalous fluctuations in some theoretical calculations is caused by technical mistakes.

The usual Bose-Einstein condensate corresponds to the accumulation of particles on the ground-state level. An important problem, considered in the review, is whether it would be admissible to create nonground-state condensates. A positive answer is given and it is explained how this could be done and what would be the features of such condensates.

Throughout the paper, the system of units is employed, where the Planck constant $\hbar=1$ and the Boltzmann constant $k_{B}=1$.

\section{Criteria of Bose-Einstein Condensation}

\subsection{Einstein Criterion}

Bose-Einstein condensation implies macroscopic accumulation of particles on the ground-state level of a Bose system. This means that, if the number of condensed particles is $N_{0}$ and the total number of particles in the system is $N$, then Bose-Einstein condensation occurs, when $N_{0}$ is proportional to $N$. To formulate this criterion in a more precise way, it is necessary to invoke the notion of the thermodynamic limit, when the number of particles $N$, as well as the system volume $V$, tend to infinity, with their ratio remaining finite:

$$
N \rightarrow \infty, \quad V \rightarrow \infty, \quad \frac{N}{V} \rightarrow \text { const } .
$$

Then the Einstein criterion is formulated as the limiting property

$$
\lim _{N \rightarrow \infty} \frac{N_{0}}{N}>0
$$


where the thermodynamic limit (1) is assumed. This is a very general criterion that, however, does not hint on how the condensate particle number $N_{0}$ should be found.

\subsection{Yang Criterion}

The Yang criterion [41] is related to the notion of the off-diagonal long-range order related to the behavior of reduced density matrices [42]. The first-order reduced density matrix $\rho\left(\mathbf{r}, \mathbf{r}^{\prime}\right)$ defines the limit

$$
\lim _{r \rightarrow \infty} \rho(\mathbf{r}, 0)=\lim _{N \rightarrow \infty} \frac{N_{0}}{V},
$$

in which $r \equiv|\mathbf{r}|$. One says that this matrix displays the off-diagonal long-range order and Bose-Einstein condensation occurs, when

$$
\lim _{r \rightarrow \infty} \rho(\mathbf{r}, 0)>0
$$

The Yang criterion can be useful for uniform systems, but is not suitable for confined systems, where the limit of $\rho(\mathbf{r}, 0)$, as $\mathbf{r} \rightarrow \infty$, is always zero, while condensation can happen [3,9].

\subsection{Penrose-Onsager Criterion}

Penrose and Onsager [43] showed that the occurrence of condensation is reflected in the eigenvalue spectrum of the single-particle density matrix. For the latter, the eigenproblem

$$
\int \rho\left(\mathbf{r}, \mathbf{r}^{\prime}\right) \varphi_{k}\left(\mathbf{r}^{\prime}\right) d \mathbf{r}^{\prime}=n_{k} \varphi_{k}(\mathbf{r})
$$

defines the eigenfunctions $\varphi_{k}(\mathbf{r})$ and eigenvalues $n_{k}$, labelled by a quantum multi-index $k$. The largest eigenvalue

$$
N_{0} \equiv \sup _{k} n_{k}
$$

gives the number of condensed particles $N_{0}$. That is, condensation occurs, when

$$
\lim _{N \rightarrow \infty} \frac{\sup _{k} n_{k}}{N}>0
$$

This criterion is quite general and can be used for uniform as well as for nonuniform systems.

\subsection{Order Indices}

A convenient criterion can be formulated by means of the order indices for reduced density matrices [44-47]. Order indices can be introduced for any operators possessing a norm and trace [48]. Let $\hat{A}$ be such an operator. Then the operator order index is defined [48] as

$$
\omega(\hat{A}) \equiv \frac{\log \|\hat{A}\|}{\log |\operatorname{Tr} \hat{A}|},
$$

where the logarithm can be taken to any base. Considering $\hat{\rho}_{1} \equiv\left[\rho\left(\mathbf{r}, \mathbf{r}^{\prime}\right)\right]$ as a matrix with respect to the spatial variables results in the order index

$$
\omega\left(\hat{\rho}_{1}\right) \equiv \frac{\log || \hat{\rho}_{1} \|}{\log \left|\operatorname{Tr} \rho_{1}\right|} .
$$


Using the expressions

$$
\left\|\hat{\rho}_{1}\right\|=\sup _{k} n_{k}=N_{0}, \quad \operatorname{Tr} \hat{\rho}_{1}=N,
$$

yields the order index for the density matrix

$$
\omega\left(\hat{\rho}_{1}\right)=\frac{\log N_{0}}{\log N} .
$$

This order index makes it possible to give the classification of different types of order:

$$
\begin{array}{ll}
\omega\left(\hat{\rho}_{1}\right) \leq 0 & \text { (no order }), \\
0<\omega\left(\hat{\rho}_{1}\right)<1 & \text { (mid }- \text { range order }), \\
\omega\left(\hat{\rho}_{1}\right)=1 & (\text { long }- \text { range order }) .
\end{array}
$$

The latter corresponds to Bose-Einstein condensation, when

$$
\lim _{N \rightarrow \infty} \omega\left(\hat{\rho}_{1}\right)=1,
$$

in agreement with the previous criteria. Generally, there can exist Bose systems with mid-range order [45-48]. In such systems there is no Bose-Einstein condensate but there happens a quasiordered state that can be called quasicondensate [39].

The order indices are useful in studying confined systems. But for confined systems, the notion of thermodynamic limit is to be generalized. For this purpose, one has to consider extensive observable quantities [49,50]. Let $A_{N}$ be such an observable quantity for a system of $N$ particles. The most general form of the thermodynamic limit can be given $[12,51]$ as the limiting condition

$$
N \rightarrow \infty, \quad A_{N} \rightarrow \infty, \quad \frac{A_{N}}{N} \rightarrow \text { const } .
$$

Similar conditions with respect to the system ground-state energy imply the system thermodynamic stability [52].

\subsection{Condensate Existence}

The condensation criteria show that Bose-Einstein condensation imposes the following restriction on the behavior of the density-matrix eigenvalues $n_{k}$. Recall that, by its definition, $n_{k}$ means the particle distribution over the quantum multi-indices $k$. According to Eqs. (6) and (7), one has

$$
\frac{1}{\sup _{k} n_{k}} \propto \frac{1}{N} \rightarrow 0 \quad(N \rightarrow \infty) .
$$

If condensation occurs into the state labelled by the multi-index $k_{0}$, so that

$$
\sup _{k} n_{k}=n_{k_{0}}
$$

then the condensation condition [12] is valid:

$$
\lim _{k \rightarrow k_{0}} \frac{1}{n_{k}}=0 \quad(N \rightarrow \infty) .
$$

Writing $N \rightarrow \infty$, implies, as usual, the thermodynamic limit in one of the forms, either as in Eq. (1) or as in Eq. (13). 


\section{Gauge Symmetry Breaking}

\subsection{Gauge Symmetry}

The global gauge symmetry $U(1)$ for a Hamiltonian $H[\psi]$, which is a functional of the field operator $\psi$, means that this Hamiltonian is invariant under the gauge transformation

$$
\psi(\mathbf{r}) \rightarrow \psi(\mathbf{r}) e^{i \alpha}
$$

where $\alpha$ is a real number. That is,

$$
H\left[\psi e^{i \alpha}\right]=H[\psi] .
$$

Here and in what follows, the time dependence of field operators is assumed but is not shown explicitly, when it is not important and cannot lead to confusion.

The field operator can always be decomposed into an expansion

$$
\psi(\mathbf{r})=\sum_{k} a_{k} \varphi_{k}(\mathbf{r})
$$

over an orthonormal complete basis. Though, in general, the basis can be arbitrary, for what follows, it is important to choose the natural basis, composed of natural orbitals [42]. By definition, the basis is natural if and only if it is composed of the eigenfunctions of the single-particle density matrix, defined by the eigenproblem (5). Then the eigenvalues $n_{k}$ describe the particle distribution over the quantum indices $k$.

Bose-Einstein condensation can occur not to any state but only into one of the states of the natural basis, that is, into one of the natural orbitals. Denoting the related natural orbital by $\varphi_{0}(\mathbf{r})$, one can write

$$
\psi(\mathbf{r})=\psi_{0}(\mathbf{r})+\psi_{1}(\mathbf{r})
$$

separating the part corresponding to condensate,

$$
\psi_{0}(\mathbf{r}) \equiv a_{0} \varphi_{0}(\mathbf{r})
$$

from the part related to uncondensed particles,

$$
\psi_{1}(\mathbf{r})=\sum_{k \neq 0} a_{k} \varphi_{k}(\mathbf{r})
$$

By construction, the condensate part is orthogonal to that of uncondensed particles:

$$
\int \psi_{0}^{\dagger}(\mathbf{r}) \psi_{1}(\mathbf{r}) d \mathbf{r}=0
$$

which follows from the orthogonality of natural orbitals. And by the definition of the natural orbitals as eigenfunctions of the single-particle density matrix, the quantum-number conservation condition

$$
\left\langle a_{k}^{\dagger} a_{p}\right\rangle=\delta_{k p}\left\langle a_{k}^{\dagger} a_{k}\right\rangle
$$

is valid. Because of the latter, one has the particular form of the quantum conservation condition

$$
\left\langle\psi_{0}^{\dagger}(\mathbf{r}) \psi_{1}(\mathbf{r})\right\rangle=0 \text {. }
$$


The number-operator for condensed particles is

$$
\hat{N}_{0} \equiv \int \psi_{0}^{\dagger}(\mathbf{r}) \psi_{0}(\mathbf{r}) d \mathbf{r}=a_{0}^{\dagger} a_{0}
$$

And the number-operator for uncondensed particles is

$$
\hat{N}_{1} \equiv \int \psi_{1}^{\dagger}(\mathbf{r}) \psi_{1}(\mathbf{r}) d \mathbf{r}=\sum_{k \neq 0} a_{k}^{\dagger} a_{k}
$$

So that the total number-operator reads as

$$
\hat{N}=\hat{N}_{0}+\hat{N}_{1}
$$

The number of condensed particles is the statistical average

$$
N_{0} \equiv\left\langle\hat{N}_{0}\right\rangle=\left\langle a_{0}^{\dagger} a_{0}\right\rangle
$$

According to the condensation criteria, Bose-Einstein condensate appears when

$$
\lim _{N \rightarrow \infty} \frac{\left\langle a_{0}^{\dagger} a_{0}\right\rangle}{N}>0 .
$$

Till now, no symmetry breaking has been involved in the consideration. Because of this, one could naively think that no symmetry breaking is necessary for treating Bose condensation. However, the above consideration is yet nothing but a set of definitions. To understand whether gauge symmetry breaking is compulsory for treating Bose condensation, one has to analyze the properties of the defined quantities.

\subsection{Symmetry Breaking}

There are several ways how the Hamiltonian symmetry could be broken. The oldest method is by incorporating in the description of the system an order parameter with a prescribed properties corresponding to a thermodynamic phase with the broken symmetry, as is done in mean-field approximations [32]. Another traditional way, advanced by Bogolubov [15,16], is by adding to the Hamiltonian symmetry-breaking terms, getting

$$
H_{\varepsilon}[\psi] \equiv H[\psi]+\varepsilon \Gamma[\psi]
$$

where $\langle\Gamma[\psi]\rangle_{\varepsilon} \propto N$ and $\varepsilon$ is a small number. The statistical averages, with Hamiltonian (30), are denoted as $\langle\cdots\rangle_{\varepsilon}$. Upon calculating such an average, one should take, first, the thermodynamic limit $N \rightarrow \infty$, after which the limit $\varepsilon \rightarrow 0$. The so defined averages are called quasiaverages. It is also possible to combine these two limits in one, prescribing to $\varepsilon$ a dependence on $N$ and taking the sole thermodynamic limit. The latter procedure defines thermodynamic quasiaverages [53]. Other methods of symmetry breaking are described in the review [54]. Here, for concreteness, the standard way of symmetry breaking by means of the Bogolubov quasiaverages will be used.

Spontaneous breaking of gauge symmetry occurs when

$$
\lim _{\varepsilon \rightarrow 0} \lim _{N \rightarrow \infty} \frac{1}{N} \int\left|\left\langle\psi_{0}(\mathbf{r})\right\rangle_{\varepsilon}\right|^{2} d \mathbf{r}>0
$$


This can also be rewritten as

$$
\lim _{\varepsilon \rightarrow 0} \lim _{N \rightarrow \infty} \frac{\left|\left\langle a_{0}\right\rangle_{\varepsilon}\right|^{2}}{N}>0 .
$$

By the Cauchy-Schwarz inequality,

$$
\left|\left\langle a_{0}\right\rangle_{\varepsilon}\right|^{2} \leq\left\langle a_{0}^{\dagger} a_{0}\right\rangle_{\varepsilon}
$$

for any $\varepsilon$. This means that gauge symmetry breaking yields Bose condensation.

Theorem 1. When gauge symmetry is spontaneously broken, then there exists Bose-Einstein condensate.

Proof. Spontaneous breaking of gauge symmetry corresponds to Eq. (32). In view of the Schwarz inequality (33), it follows that

$$
\lim _{\varepsilon \rightarrow 0} \lim _{N \rightarrow \infty} \frac{\left\langle a_{0}^{\dagger} a_{0}\right\rangle_{\varepsilon}}{N}>0,
$$

which implies Bose-Einstein condensation.

\subsection{Ginibre Theorem}

The Hamiltonian of a Bose system is a functional of the field operator $\psi$ that can always be represented as the sum (19) of two terms (20) and (21). Thus, Hamiltonian (30) is $H_{\varepsilon}[\psi]=H_{\varepsilon}\left[\psi_{0}, \psi_{1}\right]$. For an equilibrium system, this Hamiltonian defines the grand thermodynamic potential

$$
\Omega_{\varepsilon} \equiv-T \ln \operatorname{Tr} \exp \left\{-\beta H_{\varepsilon}\left[\psi_{0}, \psi_{1}\right]\right\},
$$

where $T$ is temperature and $\beta \equiv 1 / T$. Let us replace the operator term $\psi_{0}$ by a nonoperator quantity $\eta$, getting

$$
\Omega_{\eta \varepsilon} \equiv-T \ln \operatorname{Tr} \exp \left\{-\beta H_{\varepsilon}\left[\eta, \psi_{1}\right]\right\},
$$

and assuming that this thermodynamic potential is minimized with respect to $\eta$, so that

$$
\Omega_{\eta \varepsilon}=\inf _{x} \Omega_{x \varepsilon} .
$$

Ginibre [55] proved the following proposition.

Theorem 2. In thermodynamic limit, the thermodynamic potentials (35) and (36) coincide:

$$
\lim _{N \rightarrow \infty} \frac{\Omega_{\varepsilon}}{N}=\lim _{N \rightarrow \infty} \frac{\Omega_{\eta \varepsilon}}{N} .
$$

This theorem holds true irrespectively from whether there is Bose condensation or not. But if, when minimizing potential (36), one gets a nonzero $\eta$, then, according to condition (31), there is spontaneous gauge symmetry breaking. Hence, because of Theorem 1, Bose condensation occurs. 


\subsection{Bogolubov Theorem}

Let

$$
C_{\varepsilon}\left(\psi_{0}, \psi_{1}\right) \equiv\left\langle\ldots \psi_{0}^{\dagger} \ldots \psi_{1}^{\dagger} \ldots \psi_{0} \ldots \psi_{1}\right\rangle_{\varepsilon}
$$

be a class of correlation functions being the averages, with respect to the Hamiltonian $H_{\varepsilon}\left[\psi_{0}, \psi_{1}\right]$, of the normal products of the field operators (20) and (21). And let

$$
C_{\varepsilon}\left(\eta, \psi_{1}\right) \equiv\left\langle\ldots \eta^{*} \ldots \psi_{1}^{\dagger} \ldots \eta \ldots \psi_{1}\right\rangle_{\eta \varepsilon}
$$

be a class of correlation functions being the averages, with respect to the Hamiltonian $H_{\varepsilon}\left[\eta, \psi_{1}\right]$, of the normal products of the field-operator terms, where the operators $\psi_{0}$ have been replaced by a nonoperator quantity $\eta$ that minimizes the thermodynamic potential (36). Then the Bogolubov theorem [16] holds.

Theorem 3. In thermodynamic limit, the corresponding correlation functions from classes (39) and (40) coincide:

$$
\lim _{N \rightarrow \infty} C_{\varepsilon}\left(\psi_{0}, \psi_{1}\right)=\lim _{N \rightarrow \infty} C_{\varepsilon}\left(\eta, \psi_{1}\right) .
$$

As particular consequences from this theorem, it follows that

$$
\begin{gathered}
\lim _{\varepsilon \rightarrow 0} \lim _{N \rightarrow \infty} \frac{1}{N} \int\left\langle\psi_{0}^{\dagger}(\mathbf{r}) \psi_{0}(\mathbf{r})\right\rangle_{\varepsilon} d \mathbf{r}=\lim _{N \rightarrow \infty} \frac{1}{N} \int|\eta(\mathbf{r})|^{2} d \mathbf{r}, \\
\lim _{\varepsilon \rightarrow 0} \lim _{N \rightarrow \infty}\left\langle\psi_{0}(\mathbf{r})\right\rangle_{\varepsilon}=\eta(\mathbf{r}) .
\end{gathered}
$$

Invoking the conservation condition (24) yields

$$
\begin{gathered}
\lim _{\varepsilon \rightarrow 0} \lim _{N \rightarrow \infty}\left\langle\psi_{1}(\mathbf{r})\right\rangle_{\varepsilon}=0, \\
\lim _{\varepsilon \rightarrow 0} \lim _{N \rightarrow \infty}\langle\psi(\mathbf{r})\rangle_{\varepsilon}=\eta(\mathbf{r}) .
\end{gathered}
$$

Hence, if $\eta$ is not zero, the spontaneous gauge symmetry breaking takes place. Respectively, Bose condensation occurs. This important consequence of the Bogolubov theorem can be formulated as the following proposition.

Theorem 4. Spontaneous gauge symmetry breaking implies Bose-Einstein condensation:

$$
\lim _{\varepsilon \rightarrow 0} \lim _{N \rightarrow \infty} \frac{\left|\left\langle a_{0}\right\rangle_{\varepsilon}\right|^{2}}{N}=\lim _{\varepsilon \rightarrow 0} \lim _{N \rightarrow \infty} \frac{\left\langle a_{0}^{\dagger} a_{0}\right\rangle_{\varepsilon}}{N} .
$$

\subsection{Roepstorff Theorem}

The above theorems show that spontaneous gauge symmetry breaking is a sufficient condition for Bose-Einstein condensation. The fact that the former is also the necessary condition for the latter was, first, proved by Roepstorff [56] and recently the proof was polished in Refs. [57,58].

Theorem 5. Bose-Einstein condensation implies spontaneous gauge symmetry breaking:

$$
\lim _{N \rightarrow \infty} \frac{\left\langle a_{0}^{\dagger} a_{0}\right\rangle}{N} \leq \lim _{\varepsilon \rightarrow 0} \lim _{N \rightarrow \infty} \frac{\left|\left\langle a_{0}\right\rangle_{\varepsilon}\right|^{2}}{N} .
$$

In the left-hand side of inequality (45), the average is taken without explicitly breaking the gauge symmetry. Combining theorems 4 and 5 leads to the following conclusion:

Conclusion. Spontaneous gauge symmetry breaking is the necessary and sufficient condition for Bose-Einstein condensation. 


\section{General Self-Consistent Approach}

\subsection{Representative Ensembles}

A statistical ensemble is a pair $\{\mathcal{F}, \hat{\rho}\}$ of the space of microstates $\mathcal{F}$ and a statistical operator $\hat{\rho}$. Defining the statistical operator, it is necessary to take into account all conditions and constraints that uniquely describe the considered statistical system. This requirement was emphasized by Gibbs [59,60] and Ter Haar [61,62]. Such an ensemble is termed a representative ensemble. The general formulation of the representative ensembles and their properties have been given in Refs. $[54,63,64]$.

Constraints, imposed on the system, can be represented as the statistical averages of condition operators $\hat{C}_{i}$, with $i=1,2, \ldots$ being the index enumerating the condition operators. This gives the set of statistical conditions

$$
\left\langle\hat{C}_{i}\right\rangle=C_{i}
$$

Taking into account the latter defines the grand Hamiltonian

$$
H=\hat{H}+\sum_{i} \lambda_{i} \hat{C}_{i}
$$

in which $\hat{H}$ is the energy operator and $\lambda_{i}$ are Lagrange multipliers guaranteeing the validity of conditions (46).

\subsection{Bogolubov Shift}

The most convenient way of gauge symmetry breaking for Bose systems is by means of the Bogolubov shift [16] of the field operator, when the field operator $\psi$ of a system without condensate is replaced by the field operator

$$
\hat{\psi}(\mathbf{r})=\eta(\mathbf{r})+\psi_{1}(\mathbf{r})
$$

in which $\eta(\mathbf{r})$ is the condensate wave function and the second term is the field operator of uncondensed particles. The latter is a Bose operator, with the standard commutation relations

$$
\left[\psi_{1}(\mathbf{r}), \psi_{1}^{\dagger}(\mathbf{r})\right]=\delta\left(\mathbf{r}-\mathbf{r}^{\prime}\right)
$$

It is important to remember that the Fock space $\mathcal{F}(\psi)$, generated by the operator $\psi$, is orthogonal to the Fock space $\mathcal{F}\left(\psi_{1}\right)$, generated by the operator $\psi_{1}$, so that after the Bogolubov shift (48) it is necessary to work in the space $\mathcal{F}\left(\psi_{1}\right)$. Mathematical details can be found in Ref. [65].

Similarly to property (22), the condensate wave function is orthogonal to the field operator of uncondensed particles:

$$
\int \eta^{*}(\mathbf{r}) \psi_{1}(\mathbf{r}) d \mathbf{r}=0
$$

The quantum-number conservation condition, analogous to Eqs. (24) and (43), takes the form

$$
\left\langle\psi_{1}(\mathbf{r})\right\rangle=0
$$

Then Eq. (48) yields

$$
\langle\hat{\psi}(\mathbf{r})\rangle=\eta(\mathbf{r})
$$

which shows that the condensate function plays the role of an order parameter. 
The condensate function is normalized to the number of condensed particles

$$
N_{0}=\int|\eta(\mathbf{r})|^{2} d \mathbf{r} .
$$

The number of uncondensed particles gives another normalization condition

$$
N_{1}=\left\langle\hat{N}_{1}\right\rangle \text {, }
$$

where the number operator $\hat{N}_{1}$ is as in Eq. (26). The total number operator

$$
\hat{N} \equiv \int \hat{\psi}^{\dagger}(\mathbf{r}) \hat{\psi}(\mathbf{r}) d \mathbf{r}=N_{0}+\hat{N}_{1}
$$

defines the total number of particles

$$
N=\langle\hat{N}\rangle=N_{0}+N_{1} .
$$

In the Bogolubov representation of the field operator (48), the condensate function and the field operator of uncondensed particles are two independent variables, orthogonal to each other.

\subsection{Grand Hamiltonian}

The general self-consistent theory to be presented in this and in the following sections, is based on Refs. [63-71], where all details can be found.

In order to define a representative ensemble, one has to keep in mind the normalization conditions (52) and (53). The quantum-number conservation condition (50) is another restriction that is necessary to take into account. The latter equation can be rewritten in the standard form of a statistical condition by introducing the operator

$$
\hat{\Lambda} \equiv \int\left[\lambda(\mathbf{r}) \psi_{1}^{\dagger}(\mathbf{r})+\lambda^{*}(\mathbf{r}) \psi_{1}(\mathbf{r})\right] d \mathbf{r},
$$

in which $\lambda(\mathbf{r})$ is a complex function that accomplishes the role of a Lagrange multiplier guaranteeing the validity of the conservation condition (50). For this purpose, it is sufficient [71] to choose $\lambda(\mathbf{r})$ such that to kill in the Grand Hamiltonian the terms linear in $\psi_{1}(\mathbf{r})$. The conservation condition (50) can be represented as

$$
\langle\hat{\Lambda}\rangle=0 .
$$

Taking into account the given statistical conditions (52), (53), and (57) prescribes the form of the grand Hamiltonian

$$
H=\hat{H}-\mu_{0} N_{0}-\mu_{1} \hat{N}_{1}-\hat{\Lambda},
$$

in which $\mu_{0}$ and $\mu_{1}$ are the related Lagrange multipliers and $\hat{H}=\hat{H}\left[\eta, \psi_{1}\right]$ is the energy operator. The multiplier $\mu_{0}$ has the meaning of the condensate chemical potential and $\mu_{1}$ can be called the chemical potential of uncondensed particles.

The Hamiltonian average can be represented as

$$
\langle H\rangle=\langle\hat{H}\rangle-\mu N,
$$

with $\mu$ being the system chemical potential. Then, from Eq. (58), it follows that the chemical potential is

$$
\mu=\mu_{0} n_{0}+\mu_{1} n_{1},
$$


where the fractions of condensed and uncondensed particles,

$$
n_{0} \equiv \frac{N_{0}}{N}, \quad n_{1} \equiv \frac{N_{1}}{N}
$$

are introduced.

It is necessary to stress that the number of Lagrange multipliers in the grand Hamiltonian has to be equal to the number of imposed statistical conditions. Only then the statistical ensemble will be representative. In the other case, the system would not be uniquely defined. Here, there are three conditions, the normalization conditions (52) and (53) and the conservation condition (57).

It is easy to show that the multipliers $\mu_{0}$ and $\mu_{1}$ do not need to coincide. To this end, let us consider the thermodynamic stability condition requiring the extremization of the system free energy $F=F\left(T, V, N_{0}, N_{1}\right)$, that is, $\delta F=0$. This gives

$$
\delta F=\frac{\partial F}{\partial N_{0}} \delta N_{0}+\frac{\partial F}{\partial N_{1}} \delta N_{1}=0
$$

Substituting here

$$
\mu_{0}=\frac{\partial F}{\partial N_{0}}, \quad \mu_{1}=\frac{\partial F}{\partial N_{1}}
$$

transforms Eq. (62) to the equation

$$
\mu_{0} \delta N_{0}+\mu_{1} \delta N_{1}=0
$$

The total number of particles $N=N_{0}+N_{1}$ is assumed to be fixed, so that $\delta N=0$ and $\delta N_{0}=-\delta N_{1}$. Then Eq. (64) reduces to the relation

$$
\left(\mu_{0}-\mu_{1}\right) \delta N_{1}=0
$$

If $N_{1}$ were arbitrary, then one would have the equivalence of the multipliers $\mu_{0}$ and $\mu_{1}$. However, the number of uncondensed particles $N_{1}$ is fixed for each fixed $T, V$, and $N$. That is, $\delta N_{1}=0$ and Eq. (65) is satisfied for any multipliers. Hence the multipliers $\mu_{0}$ and $\mu_{1}$ do not have to be equal.

It would be possible to say that $N_{0}$ is fixed for each given $T, V, N$. But, clearly, this is the same as to say that $N_{1}$ is fixed. In any case, there always exist two normalization conditions requiring to introduce two related Lagrange multipliers.

The Hamiltonian energy operator is

$$
\begin{gathered}
\hat{H}=\int \hat{\psi}^{\dagger}(\mathbf{r})\left(-\frac{\nabla^{2}}{2 m}+U\right) \hat{\psi}(\mathbf{r}) d \mathbf{r}+ \\
+\frac{1}{2} \int \hat{\psi}^{\dagger}(\mathbf{r}) \hat{\psi}^{\dagger}\left(\mathbf{r}^{\prime}\right) \Phi\left(\mathbf{r}-\mathbf{r}^{\prime}\right) \hat{\psi}\left(\mathbf{r}^{\prime}\right) \hat{\psi}(\mathbf{r}) d \mathbf{r} d \mathbf{r}^{\prime},
\end{gathered}
$$

where $\Phi(-\mathbf{r})=\Phi(\mathbf{r})$ is a pair interaction potential and $U=U(\mathbf{r}, t)$ is an external potential that, generally, can depend on time $t$.

Substituting into the grand Hamiltonian (58) the shifted operator (48) results in the form

$$
H=\sum_{n=0}^{4} H^{(n)},
$$


whose terms are classified according to the order of the products of the field operators $\psi_{1}$. The zero-order term

$$
\begin{aligned}
H^{(0)} & =\int \eta^{*}(\mathbf{r})\left(-\frac{\nabla^{2}}{2 m}+U-\mu_{0}\right) \eta(\mathbf{r}) d \mathbf{r}+ \\
& +\frac{1}{2} \int \Phi\left(\mathbf{r}-\mathbf{r}^{\prime}\right)\left|\eta\left(\mathbf{r}^{\prime}\right)\right|^{2}|\eta(\mathbf{r})|^{2} d \mathbf{r} d \mathbf{r}^{\prime}
\end{aligned}
$$

does not contain the operators $\psi_{1}$. The first-order term

$$
H^{(1)}=0
$$

is zero because of the conservation condition (57). The second-order term is

$$
\begin{gathered}
H^{(2)}=\int \psi_{1}^{\dagger}(\mathbf{r})\left(-\frac{\nabla^{2}}{2 m}+U-\mu_{1}\right) \psi_{1}(\mathbf{r}) d \mathbf{r}+ \\
+\int \Phi\left(\mathbf{r}-\mathbf{r}^{\prime}\right)\left[|\eta(\mathbf{r})|^{2} \psi_{1}^{\dagger}\left(\mathbf{r}^{\prime}\right) \psi_{1}\left(\mathbf{r}^{\prime}\right)+\eta^{*}(\mathbf{r}) \eta\left(\mathbf{r}^{\prime}\right) \psi_{1}^{\dagger}\left(\mathbf{r}^{\prime}\right) \psi_{1}(\mathbf{r})+\right. \\
\left.+\frac{1}{2} \eta^{*}(\mathbf{r}) \eta^{*}\left(\mathbf{r}^{\prime}\right) \psi_{1}\left(\mathbf{r}^{\prime}\right) \psi_{1}(\mathbf{r})+\frac{1}{2} \eta(\mathbf{r}) \eta\left(\mathbf{r}^{\prime}\right) \psi_{1}^{\dagger}\left(\mathbf{r}^{\prime}\right) \psi_{1}^{\dagger}(\mathbf{r})\right] d \mathbf{r} d \mathbf{r}^{\prime}
\end{gathered}
$$

Respectively, one has the third-order term

$$
H^{(3)}=\int \Phi\left(\mathbf{r}-\mathbf{r}^{\prime}\right)\left[\eta^{*}(\mathbf{r}) \psi_{1}^{\dagger}\left(\mathbf{r}^{\prime}\right) \psi_{1}\left(\mathbf{r}^{\prime}\right) \psi_{1}(\mathbf{r})+\psi_{1}^{\dagger}(\mathbf{r}) \psi_{1}^{\dagger}\left(\mathbf{r}^{\prime}\right) \psi_{1}\left(\mathbf{r}^{\prime}\right) \eta(\mathbf{r})\right] d \mathbf{r} d \mathbf{r}^{\prime}
$$

and the fourth-order term

$$
H^{(4)}=\frac{1}{2} \int \psi_{1}^{\dagger}(\mathbf{r}) \psi_{1}^{\dagger}\left(\mathbf{r}^{\prime}\right) \Phi\left(\mathbf{r}-\mathbf{r}^{\prime}\right) \psi_{1}\left(\mathbf{r}^{\prime}\right) \psi_{1}(\mathbf{r}) d \mathbf{r} d \mathbf{r}^{\prime}
$$

\subsection{Variational Principle}

In the Heisenberg representation, field operators satisfy the Heisenberg equation involving a commutator of the operator with the system Hamiltonian. At the same time, in quantum field theory, one usually gets the equations for the field operators by extremizing an action functional $[72,73]$, which reduces to the variation of the Hamiltonian. Conditions, when these two methods are equivalent, are clarified in the following propositions.

Theorem 6. Let a field operator $\psi(\mathbf{r})$ be either Bose or Fermi operator satisfying, respectively, the commutation or anticommutation relations

$$
\left[\psi(\mathbf{r}), \psi^{\dagger}\left(\mathbf{r}^{\prime}\right)\right]_{\mp}=\delta\left(\mathbf{r}-\mathbf{r}^{\prime}\right), \quad\left[\psi(\mathbf{r}), \psi\left(\mathbf{r}^{\prime}\right)\right]_{\mp}=0,
$$

with the upper sign index being for Bose statistics while the lower, for Fermi statistics. Then for the products

$$
P_{m n} \equiv P_{m}^{+} P_{n}, \quad P_{m}^{+} \equiv \prod_{i=1}^{m} \psi^{\dagger}\left(\mathbf{r}_{i}\right), \quad P_{n} \equiv \prod_{i=1}^{n} \psi\left(\mathbf{r}_{i}^{\prime}\right),
$$

where $m$ and $n$ are real integers, one has the commutators

$$
\left[\psi(\mathbf{r}), P_{m n}\right]=\frac{\delta P_{m n}}{\delta \psi^{\dagger}(\mathbf{r})}+\left[( \pm 1)^{m+n}-1\right] P_{m n} \psi(\mathbf{r})
$$


Proof. Using the variational derivative

$$
\frac{\delta \psi^{\dagger}\left(\mathbf{r}_{i}\right)}{\delta \psi^{\dagger}(\mathbf{r})}=\delta\left(\mathbf{r}-\mathbf{r}_{i}\right)
$$

it is straightforward to find

$$
\begin{gathered}
\frac{\delta P_{1}^{+}}{\delta \psi^{\dagger}(\mathbf{r})}=\delta\left(\mathbf{r}-\mathbf{r}_{1}\right), \quad \frac{\delta P_{2}^{+}}{\delta \psi^{\dagger}(\mathbf{r})}=\delta\left(\mathbf{r}-\mathbf{r}_{1}\right) \psi^{\dagger}\left(\mathbf{r}_{2}\right) \pm \delta\left(\mathbf{r}-\mathbf{r}_{2}\right) \psi^{\dagger}\left(\mathbf{r}_{1}\right), \\
\frac{\delta P_{3}^{+}}{\delta \psi^{\dagger}(\mathbf{r})}=\delta\left(\mathbf{r}-\mathbf{r}_{1}\right) \psi^{\dagger}\left(\mathbf{r}_{2}\right) \psi^{\dagger}\left(\mathbf{r}_{3}\right) \pm \delta\left(\mathbf{r}-\mathbf{r}_{2}\right) \psi^{\dagger}\left(\mathbf{r}_{1}\right) \psi^{\dagger}\left(\mathbf{r}_{3}\right)+\delta\left(\mathbf{r}-\mathbf{r}_{3}\right) \psi^{\dagger}\left(\mathbf{r}_{1}\right) \psi^{\dagger}\left(\mathbf{r}_{2}\right)
\end{gathered}
$$

and so on. By induction, it follows that

$$
\frac{\delta P_{m}^{+}}{\delta \psi^{\dagger}(\mathbf{r})}=\sum_{j=1}^{m}( \pm 1)^{j+1} \delta\left(\mathbf{r}-\mathbf{r}_{j}\right) \prod_{i(\neq j)}^{m} \psi^{\dagger}\left(\mathbf{r}_{i}\right)
$$

Using the commutator

$$
\left[\psi(\mathbf{r}), \psi^{\dagger}\left(\mathbf{r}^{\prime}\right)\right]=\delta\left(\mathbf{r}-\mathbf{r}^{\prime}\right)+( \pm 1-1) \psi^{\dagger}\left(\mathbf{r}^{\prime}\right) \psi(\mathbf{r})
$$

we derive

$$
\begin{gathered}
{\left[\psi(\mathbf{r}), P_{1}^{+}\right]=\delta\left(\mathbf{r}-\mathbf{r}_{1}\right)+( \pm 1-1) P_{1}^{+} \psi(\mathbf{r})} \\
{\left[\psi(\mathbf{r}), P_{2}^{+}\right]=\delta\left(\mathbf{r}-\mathbf{r}_{1}\right) \psi^{\dagger}\left(\mathbf{r}_{2}\right) \pm \delta\left(\mathbf{r}-\mathbf{r}_{2}\right) \psi^{\dagger}\left(\mathbf{r}_{1}\right)} \\
{\left[\psi(\mathbf{r}), P_{3}^{+}\right]=\delta\left(\mathbf{r}-\mathbf{r}_{1}\right) \psi^{\dagger}\left(\mathbf{r}_{2}\right) \psi^{\dagger}\left(\mathbf{r}_{3}\right) \pm \delta\left(\mathbf{r}-\mathbf{r}_{2}\right) \psi^{\dagger}\left(\mathbf{r}_{1}\right) \psi^{\dagger}\left(\mathbf{r}_{3}\right)+} \\
+\delta\left(\mathbf{r}-\mathbf{r}_{3}\right) \psi^{\dagger}\left(\mathbf{r}_{1}\right) \psi^{\dagger}\left(\mathbf{r}_{2}\right)+( \pm 1-1) P_{3}^{+} \psi(\mathbf{r})
\end{gathered}
$$

and so on. From here, using Eq. (76), by induction, we get

$$
\left[\psi(\mathbf{r}), P_{m}^{+}\right]=\frac{\delta P_{m}^{+}}{\delta \psi^{\dagger}(\mathbf{r})}+\left[( \pm 1)^{m}-1\right] P_{m}^{+} \psi(\mathbf{r}) .
$$

Also, it is easy to check that

$$
\left[\psi(\mathbf{r}), P_{n}\right]=\left[( \pm 1)^{n}-1\right] P_{n} \psi(\mathbf{r}) .
$$

Then, taking into account that, for any three operators $\hat{A}, \hat{B}, \hat{C}$, the equality

$$
[\hat{A}, \hat{B} \hat{C}]=[\hat{A}, \hat{B}] \hat{C}+\hat{B}[\hat{A}, \hat{C}]
$$

is valid, we have

$$
\left[\psi(\mathbf{r}), P_{m n}\right]=\left[\psi(\mathbf{r}), P_{m}^{+}\right] P_{n}+P_{m}^{+}\left[\psi(\mathbf{r}), P_{n}\right] .
$$

Substituting here Eqs. (77) and (78) gives the required Eq. (75).

Theorem 7. Let $\hat{F}\left[P_{m n}\right]$ be a linear functional of the products defined in Eq. (74). And let the linear combination

$$
\hat{F}=\sum_{m n} c_{m n} \hat{F}\left[P_{m n}\right]
$$


contain only such functionals for which, in the case of Bose statistics, $m$ and $n$ are arbitrary while, for the case of Fermi statistics, $m+n$ is even. Then

$$
[\psi(\mathbf{r}), \hat{F}]=\frac{\delta \hat{F}}{\delta \psi^{\dagger}(\mathbf{r})} .
$$

Proof. The proof is straightforward, following immediately from Eq. (75).

The latter theorem shows that for a large class of functionals the commutator with the field operator is equivalent to the variational derivative. The operators of observable quantities are in this class, as well as Hamiltonians. This is because for Fermi systems, the field operators enter the observables always in pairs, which is necessary for spin conservation. This is why the Heisenberg equations for the field operators can be written in two equivalent ways, in the form of a commutator, as in the left-hand side of Eq. (80), or in the form of a variational derivative, as in the right-hand side of that equation. Note that the standard form of many phenomenological evolution equations also involves variational derivatives [74,75].

\subsection{Evolution Equations}

With the grand Hamiltonian (58), the evolution equations for the field variables $\eta$ and $\psi_{1}$ read as

$$
i \frac{\partial}{\partial t} \eta(\mathbf{r}, t)=\frac{\delta H}{\delta \eta^{*}(\mathbf{r}, t)}
$$

for the condensate function, and as

$$
i \frac{\partial}{\partial t} \psi_{1}(\mathbf{r}, t)=\frac{\delta H}{\delta \psi_{1}^{\dagger}(\mathbf{r}, t)},
$$

for the field operator of uncondensed particles. Recall that, in view of Theorem 7 ,

$$
\frac{\delta H}{\delta \psi_{1}^{\dagger}(\mathbf{r}, t)}=\left[\psi_{i}(\mathbf{r}, t), H\right]
$$

Invoking expression (67) of the grand Hamiltonian gives the equation

$$
\begin{aligned}
& i \frac{\partial}{\partial t} \eta(\mathbf{r}, t)=\left(-\frac{\nabla^{2}}{2 m}+U-\mu_{0}\right) \eta(\mathbf{r}, t)+ \\
& +\int \Phi\left(\mathbf{r}-\mathbf{r}^{\prime}\right)\left[\hat{X}_{0}\left(\mathbf{r}, \mathbf{r}^{\prime}\right)+\hat{X}\left(\mathbf{r}, \mathbf{r}^{\prime}\right)\right] d \mathbf{r}^{\prime}
\end{aligned}
$$

in which the notations are introduced:

$$
\begin{gathered}
\hat{X}_{0}\left(\mathbf{r}, \mathbf{r}^{\prime}\right) \equiv \eta^{*}\left(\mathbf{r}^{\prime}\right) \eta\left(\mathbf{r}^{\prime}\right) \eta(\mathbf{r}) \\
\hat{X}\left(\mathbf{r}, \mathbf{r}^{\prime}\right) \equiv \psi_{1}^{\dagger}\left(\mathbf{r}^{\prime}\right) \psi_{1}\left(\mathbf{r}^{\prime}\right) \eta(\mathbf{r})+\psi_{1}^{\dagger}\left(\mathbf{r}^{\prime}\right) \eta\left(\mathbf{r}^{\prime}\right) \psi_{1}(\mathbf{r})+ \\
+\eta^{*}\left(\mathbf{r}^{\prime}\right) \psi_{1}\left(\mathbf{r}^{\prime}\right) \psi_{1}(\mathbf{r})+\psi_{1}^{\dagger}\left(\mathbf{r}^{\prime}\right) \psi_{1}\left(\mathbf{r}^{\prime}\right) \psi_{1}(\mathbf{r}) .
\end{gathered}
$$

In these expressions, for brevity, the explicit dependence on time is not shown. 
Equation (82) yields the equation for the field operator of uncondensed particles:

$$
\begin{gathered}
i \frac{\partial}{\partial t} \psi_{1}(\mathbf{r}, t)=\left(-\frac{\nabla^{2}}{2 m}+U-\mu_{1}\right) \psi_{1}(\mathbf{r}, t)+ \\
+\int \Phi\left(\mathbf{r}-\mathbf{r}^{\prime}\right)\left[\hat{X}_{1}\left(\mathbf{r}, \mathbf{r}^{\prime}\right)+\hat{X}\left(\mathbf{r}, \mathbf{r}^{\prime}\right)\right] d \mathbf{r}^{\prime}
\end{gathered}
$$

where

$$
\hat{X}_{1}\left(\mathbf{r}, \mathbf{r}^{\prime}\right) \equiv \eta^{*}\left(\mathbf{r}^{\prime}\right) \eta\left(\mathbf{r}^{\prime}\right) \psi_{1}(\mathbf{r})+\eta^{*}\left(\mathbf{r}^{\prime}\right) \psi_{1}\left(\mathbf{r}^{\prime}\right) \eta(\mathbf{r})+\psi_{1}^{\dagger}\left(\mathbf{r}^{\prime}\right) \eta\left(\mathbf{r}^{\prime}\right) \eta(\mathbf{r}) .
$$

An equation for the condensate function follows from averaging Eq. (81), with the standard notation for a statistical average of an operator $\hat{A}$ as

$$
\langle\hat{A}(t)\rangle \equiv \operatorname{Tr} \hat{\rho}(0) \hat{A}(t)
$$

where $\hat{\rho}(0)$ is the statistical operator at the initial time $t=0$. So that the condensate-function equation is

$$
i \frac{\partial}{\partial t} \eta(\mathbf{r}, t)=\left\langle\frac{\delta H}{\delta \eta^{*}(\mathbf{r}, t)}\right\rangle .
$$

Averaging the right-hand side of Eq. (83), we shall need the notations for the single-particle density matrix

$$
\rho_{1}\left(\mathbf{r}, \mathbf{r}^{\prime}\right) \equiv\left\langle\psi_{1}^{\dagger}\left(\mathbf{r}^{\prime}\right) \psi_{1}(\mathbf{r})\right\rangle
$$

and the anomalous density matrix

$$
\sigma_{1}\left(\mathbf{r}, \mathbf{r}^{\prime}\right) \equiv\left\langle\psi_{1}\left(\mathbf{r}^{\prime}\right) \psi_{1}(\mathbf{r})\right\rangle
$$

The density of condensed particles is

$$
\rho_{0}(\mathbf{r}) \equiv|\eta(\mathbf{r})|^{2}
$$

while the density of uncondensed particles is

$$
\rho_{1}(\mathbf{r}) \equiv \rho_{1}(\mathbf{r}, \mathbf{r})=\left\langle\psi_{1}^{\dagger}(\mathbf{r}) \psi_{1}(\mathbf{r})\right\rangle
$$

The diagonal element of the anomalous density matrix,

$$
\sigma_{1}(\mathbf{r}) \equiv \sigma_{1}(\mathbf{r}, \mathbf{r})=\left\langle\psi_{1}(\mathbf{r}) \psi_{1}(\mathbf{r})\right\rangle
$$

defines the density of pair-correlated particles as $\left|\sigma_{1}(\mathbf{r})\right|$. The total density of particles in the system is the sum

$$
\rho(\mathbf{r})=\rho_{0}(\mathbf{r})+\rho_{1}(\mathbf{r}) .
$$

Also, we shall need the notation for the anomalous triple correlator

$$
\xi\left(\mathbf{r}, \mathbf{r}^{\prime}\right) \equiv\left\langle\psi_{1}^{\dagger}\left(\mathbf{r}^{\prime}\right) \psi_{1}\left(\mathbf{r}^{\prime}\right) \psi_{1}(\mathbf{r})\right\rangle .
$$

Employing these notations gives

$$
\begin{gathered}
\hat{X}_{0}\left(\mathbf{r}, \mathbf{r}^{\prime}\right)=\rho_{0}\left(\mathbf{r}^{\prime}\right) \eta(\mathbf{r}) \\
\left\langle\hat{X}\left(\mathbf{r}, \mathbf{r}^{\prime}\right)\right\rangle=\rho_{1}\left(\mathbf{r}^{\prime}\right) \eta(\mathbf{r})+\rho_{1}\left(\mathbf{r}, \mathbf{r}^{\prime}\right) \eta\left(\mathbf{r}^{\prime}\right)+\sigma_{1}\left(\mathbf{r}, \mathbf{r}^{\prime}\right) \eta^{*}\left(\mathbf{r}^{\prime}\right)+\xi\left(\mathbf{r}, \mathbf{r}^{\prime}\right) .
\end{gathered}
$$


Finally, Eqs. (83) and (87) result in the equation for the condensate function

$$
\begin{gathered}
i \frac{\partial}{\partial t} \eta(\mathbf{r}, t)=\left(-\frac{\nabla^{2}}{2 m}+U-\mu_{0}\right) \eta(\mathbf{r}, t)+ \\
+\int \Phi\left(\mathbf{r}-\mathbf{r}^{\prime}\right)\left[\rho\left(\mathbf{r}^{\prime}\right) \eta(\mathbf{r})+\rho_{1}\left(\mathbf{r}, \mathbf{r}^{\prime}\right) \eta\left(\mathbf{r}^{\prime}\right)+\sigma_{1}\left(\mathbf{r}, \mathbf{r}^{\prime}\right) \eta^{*}\left(\mathbf{r}^{\prime}\right)+\xi\left(\mathbf{r}, \mathbf{r}^{\prime}\right)\right] d \mathbf{r}^{\prime} .
\end{gathered}
$$

Equations for the densities can be obtained from the above equations, with introducing the condensate density of current

$$
\mathbf{j}_{0}(\mathbf{r}, t) \equiv-\frac{i}{2 m}\left[\eta^{*}(\mathbf{r}) \nabla \eta(\mathbf{r})-\eta(\mathbf{r}) \nabla \eta^{*}(\mathbf{r})\right]
$$

and the current density of uncondensed particles

$$
\mathbf{j}_{1}(\mathbf{r}, t) \equiv-\frac{i}{2 m}\left\langle\psi_{1}^{\dagger}(\mathbf{r}) \nabla \psi_{1}(\mathbf{r})-\left[\nabla \psi_{1}^{\dagger}(\mathbf{r})\right] \psi_{1}(\mathbf{r})\right\rangle
$$

And let us also define the source term

$$
\Gamma(\mathbf{r}, t) \equiv i \int \Phi\left(\mathbf{r}-\mathbf{r}^{\prime}\right)\left[\Xi^{*}\left(\mathbf{r}, \mathbf{r}^{\prime}\right)-\Xi\left(\mathbf{r}, \mathbf{r}^{\prime}\right)\right] d \mathbf{r}^{\prime},
$$

with the anomalous correlation function

$$
\Xi\left(\mathbf{r}, \mathbf{r}^{\prime}\right) \equiv \eta^{*}(\mathbf{r})\left[\eta^{*}\left(\mathbf{r}^{\prime}\right) \sigma_{1}\left(\mathbf{r}, \mathbf{r}^{\prime}\right)+\xi\left(\mathbf{r}, \mathbf{r}^{\prime}\right)\right] .
$$

Then we get the continuity equations for the condensate,

$$
\frac{\partial}{\partial t} \rho_{0}(\mathbf{r}, t)+\nabla \cdot \mathbf{j}_{0}(\mathbf{r}, t)=\Gamma(\mathbf{r}, t)
$$

and for uncondensed particles,

$$
\frac{\partial}{\partial t} \rho_{1}(\mathbf{r}, t)+\nabla \cdot \mathbf{j}_{1}(\mathbf{r}, t)=-\Gamma(\mathbf{r}, t) .
$$

The total density (93) satisfies the continuity equation

$$
\frac{\partial}{\partial t} \rho(\mathbf{r}, t)+\nabla \cdot \mathbf{j}(\mathbf{r}, t)=0
$$

with the total density of current

$$
\mathbf{j}(\mathbf{r}, t)=\mathbf{j}_{0}(\mathbf{r}, t)+\mathbf{j}_{1}(\mathbf{r}, t) .
$$

For the anomalous diagonal average (92), we find the equation

$$
i \frac{\partial}{\partial t} \sigma_{1}(\mathbf{r}, t)=2 K(\mathbf{r}, t)+2\left(U-\mu_{1}\right) \sigma_{1}(\mathbf{r}, t)+2 \int \Phi\left(\mathbf{r}-\mathbf{r}^{\prime}\right) S\left(\mathbf{r}, \mathbf{r}^{\prime}, t\right) d \mathbf{r}^{\prime}
$$

where the average anomalous kinetic-energy density is defined as

$$
K(\mathbf{r}, t)=-\frac{1}{2}\left\langle\frac{\nabla^{2} \psi_{1}(\mathbf{r})}{2 m} \psi_{1}(\mathbf{r})+\psi_{1}(\mathbf{r}) \frac{\nabla^{2} \psi_{1}(\mathbf{r})}{2 m}\right\rangle
$$

and where we use the notation

$$
\begin{gathered}
S\left(\mathbf{r}, \mathbf{r}^{\prime}, t\right)=\eta(\mathbf{r}) \eta\left(\mathbf{r}^{\prime}\right) \rho_{1}\left(\mathbf{r}, \mathbf{r}^{\prime}\right)+\eta^{*}\left(\mathbf{r}^{\prime}\right) \eta(\mathbf{r}) \sigma_{1}\left(\mathbf{r}, \mathbf{r}^{\prime}\right)+\eta^{*}\left(\mathbf{r}^{\prime}\right) \eta\left(\mathbf{r}^{\prime}\right) \sigma_{1}(\mathbf{r})+ \\
+\eta(\mathbf{r}) \xi\left(\mathbf{r}, \mathbf{r}^{\prime}\right)+\eta\left(\mathbf{r}^{\prime}\right)\left\langle\psi_{1}^{\dagger}\left(\mathbf{r}^{\prime}\right) \psi_{1}(\mathbf{r}) \psi_{1}(\mathbf{r})\right\rangle+ \\
+\eta^{*}\left(\mathbf{r}^{\prime}\right)\left\langle\psi_{1}\left(\mathbf{r}^{\prime}\right) \psi_{1}(\mathbf{r}) \psi_{1}(\mathbf{r})\right\rangle+\left\langle\psi_{1}^{\dagger}\left(\mathbf{r}^{\prime}\right) \psi_{1}\left(\mathbf{r}^{\prime}\right) \psi_{1}(\mathbf{r}) \psi_{1}(\mathbf{r})\right\rangle+\left[\eta^{2}(\mathbf{r})+\sigma_{1}(\mathbf{r})\right] \delta\left(\mathbf{r}-\mathbf{r}^{\prime}\right) .
\end{gathered}
$$




\section{$5 \quad$ Superfluidity in Quantum Systems}

\subsection{Superfluid Fraction}

One of the most important features of Bose-condensed systems is superfluidity. Therefore it is necessary to have a general definition for calculating the superfluid fraction. Probably, the most general such a definition is by identifying the superfluid fraction as the fraction of particles nontrivially responding to a velocity boost.

The systems Hamiltonian $H=H[\hat{\psi}]$ is a functional of the field operator $\hat{\psi}$. The operator of momentum is

$$
\hat{\mathbf{P}} \equiv \int \hat{\psi}^{\dagger}(\mathbf{r}) \hat{\mathbf{p}} \hat{\psi}(\mathbf{r}) d \mathbf{r}
$$

where $\hat{\mathbf{p}} \equiv-i \nabla$.

Boosting the system with a velocity $\mathbf{v}$ leads to the Galilean transformation of the field operators in the laboratory frame

$$
\hat{\psi}_{v}(\mathbf{r}, t)=\hat{\psi}(\mathbf{r}-\mathbf{v} t) \exp \left\{i\left(m \mathbf{v} \cdot \mathbf{r}-\frac{m v^{2}}{2} t\right)\right\}
$$

expressed through the field operators $\hat{\psi}$ in the frame accompanying the moving system. Then the operator of momentum in the frame at rest,

$$
\hat{\mathbf{P}}_{v} \equiv \int \psi_{v}^{\dagger}(\mathbf{r}) \hat{\mathbf{p}} \hat{\psi}_{v}(\mathbf{r}) d \mathbf{r}
$$

transforms into

$$
\hat{\mathbf{P}}_{v}=\int \hat{\psi}^{\dagger}(\mathbf{r})(\mathbf{p}+m \mathbf{v}) \hat{\psi}(\mathbf{r}) d \mathbf{r}=\hat{\mathbf{P}}+m \mathbf{v} \hat{N}
$$

Since

$$
\frac{(\hat{\mathbf{p}}+m \mathbf{v})^{2}}{2 m}=\frac{\hat{\mathbf{p}}^{2}}{2 m}+\mathbf{v} \cdot \hat{\mathbf{p}}+\frac{m v^{2}}{2},
$$

the Hamiltonian $H_{v}=H\left[\hat{\psi}_{v}\right]$ for the moving system becomes

$$
H_{v}=H+\int \hat{\psi}^{\dagger}(\mathbf{r})\left(\mathbf{v} \cdot \hat{\mathbf{p}}+\frac{m v^{2}}{2}\right) \hat{\psi}(\mathbf{r}) d \mathbf{r} .
$$

The generalized superfluid fraction is defined through the ratio

$$
n_{s}(\mathbf{v}) \equiv \frac{\frac{\partial}{\partial \mathbf{v}} \cdot\left\langle\hat{\mathbf{P}}_{v}\right\rangle_{v}}{\left\langle\frac{\partial}{\partial \mathbf{v}} \cdot \hat{\mathbf{P}}_{v}\right\rangle_{v}}
$$

in which the statistical averages $\langle\cdots\rangle_{v}$ are determined for the moving system with the Hamiltonian $H_{v}$, given in Eq. (110). This definition is valid for any system, including nonequilibrium and nonuniform systems of arbitrary statistics.

One usually defines the superfluid fraction for a system at rest, which gives

$$
n_{s} \equiv \lim _{v \rightarrow 0} n_{s}(\mathbf{v})
$$


For equilibrium systems, the statistical averages are given by the expressions

$$
\langle\hat{A}\rangle_{v} \equiv \frac{\operatorname{Tr} \hat{A} \exp \left(-\beta H_{v}\right)}{\operatorname{Tr} \exp \left(-\beta H_{v}\right)},
$$

for the moving system, and by

$$
\langle\hat{A}\rangle \equiv \frac{\operatorname{Tr} \hat{A} e^{-\beta H}}{\operatorname{Tr} e^{-\beta H}}=\lim _{v \rightarrow 0}\langle\hat{A}\rangle_{v},
$$

for the system at rest.

In the case of equilibrium systems, the derivatives over parameters can be calculated according to the formulas of Ref. [76]. Thus, we have

$$
\frac{\partial}{\partial \mathbf{v}} \cdot\left\langle\hat{\mathbf{P}}_{v}\right\rangle_{v}=\left\langle\frac{\partial}{\partial \mathbf{v}} \cdot \hat{\mathbf{P}}_{v}\right\rangle_{v}-\beta \operatorname{cov}\left(\hat{\mathbf{P}}_{v}, \frac{\partial H_{v}}{\partial \mathbf{v}}\right),
$$

where the covariance of any two operators, $\hat{A}$ and $\hat{B}$, is

$$
\operatorname{cov}(\hat{A}, \hat{B}) \equiv \frac{1}{2}\langle\hat{A} \hat{B}+\hat{B} \hat{A}\rangle_{v}-\langle\hat{A}\rangle_{v}\langle\hat{B}\rangle_{v} .
$$

¿From Eqs. (109) and (110), one has

$$
\frac{\partial}{\partial \mathbf{v}} \cdot \hat{\mathbf{P}}_{v}=3 m \hat{N}, \quad \frac{\partial H_{v}}{\partial \mathbf{v}}=\hat{\mathbf{P}}_{v} .
$$

Consequently, fraction (111) becomes

$$
n_{s}(\mathbf{v})=1-\frac{\Delta^{2}\left(\hat{\mathbf{P}}_{v}\right)}{3 m N T},
$$

where the notation for an operator dispersion

$$
\Delta^{2}\left(\hat{A}_{v}\right) \equiv\left\langle\hat{A}_{v}^{2}\right\rangle_{v}-\left\langle\hat{A}_{v}\right\rangle_{v}^{2}
$$

is used. Therefore, for fraction (112), Eq. (116) yields

$$
n_{s}=1-\frac{\Delta^{2}(\hat{\mathbf{P}})}{3 m N T},
$$

with the dispersion given as

$$
\Delta^{2}(\hat{A}) \equiv\left\langle\hat{A}^{2}\right\rangle-\langle\hat{A}\rangle^{2} .
$$

The quantity

$$
Q \equiv \frac{\Delta^{2}(\hat{\mathbf{P}})}{2 m N}
$$

describes the heat dissipated in the considered quantum system. While the dissipated heat in the classical case reads as

$$
Q_{0} \equiv \frac{3}{2} T \text {. }
$$


Hence, the superfluid fraction (117) can be represented by the expression

$$
n_{s}=1-\frac{Q}{Q_{0}}
$$

For an immovable system, the average momentum $<\hat{\mathbf{P}}>$ is zero. Then

$$
\Delta^{2}(\hat{\mathbf{P}})=\left\langle\hat{\mathbf{P}}^{2}\right\rangle \quad(\langle\hat{\mathbf{P}}\rangle=0)
$$

And the dissipated heat reduces to

$$
Q=\frac{\left\langle\hat{\mathbf{P}}^{2}\right\rangle}{2 m N}
$$

\subsection{Moment of Inertia}

Another way of defining the superfluid fraction is through the system response to rotation. The latter is connected with the angular momentum operator

$$
\hat{\mathbf{L}} \equiv \int \hat{\psi}^{\dagger}(\mathbf{r})(\mathbf{r} \times \hat{\mathbf{p}}) \hat{\psi}(\mathbf{r}) d \mathbf{r}
$$

When the system is rotated with the angular velocity $\vec{\omega}$, the related linear velocity is

$$
\mathbf{v}_{\omega} \equiv \vec{\omega} \times \mathbf{r}
$$

Then, in the laboratory frame, the angular momentum operator takes the form

$$
\hat{\mathbf{L}}_{\omega}=\int \hat{\psi}^{\dagger}(\mathbf{r})\left[\mathbf{r} \times\left(\hat{\mathbf{p}}+m \mathbf{v}_{\omega}\right)\right] \hat{\psi}(\mathbf{r}) d \mathbf{r} .
$$

This, using the equality

$$
\mathbf{r} \times(\vec{\omega} \times \mathbf{r})=r^{2} \vec{\omega}-(\vec{\omega} \cdot \mathbf{r}) \mathbf{r}
$$

gives

$$
\hat{\mathbf{L}}_{\omega}=\hat{\mathbf{L}}+m \int \hat{\psi}^{\dagger}(\mathbf{r})\left[r^{2} \vec{\omega}-(\vec{\omega} \cdot \mathbf{r}) \mathbf{r}\right] \hat{\psi}(\mathbf{r}) d \mathbf{r}
$$

The energy Hamiltonian of an immovable system can be written as the sum

$$
\hat{H}=\hat{K}+\hat{V}
$$

of the kinetic energy operator

$$
\hat{K} \equiv \int \hat{\psi}^{\dagger}(\mathbf{r}) \frac{\hat{\mathbf{p}}^{2}}{2 m} \hat{\psi}(\mathbf{r}) d \mathbf{r}
$$

and the potential energy part $\hat{V}$, respectively.

Under rotation, the potential energy part does not change, but only the kinetic part varies, so that the energy Hamiltonian of a rotating system, in the laboratory frame, becomes

$$
\hat{H}_{\omega}=\hat{K}_{\omega}+\hat{V}
$$


with the same potential energy operator $\hat{V}$. The kinetic energy operator, in the laboratory frame, can be represented $[77,78]$ by the formula

$$
\hat{K}_{\omega}=\int \hat{\psi}^{\dagger}(\mathbf{r}) \frac{\left(\hat{\mathbf{p}}+m \mathbf{v}_{\omega}\right)^{2}}{2 m} \hat{\psi}(\mathbf{r}) d \mathbf{r}
$$

In the rotating frame, where the system is at rest, the kinetic energy operator can be obtained from Eq. (129) with replacing $\vec{\omega}$ by $-\vec{\omega}$ and, respectively, replacing $\mathbf{v}_{\omega}$ by $-\mathbf{v}_{\omega}$. Using the relations

$$
(\vec{\omega} \times \mathbf{r})^{2}=\omega^{2} r^{2}-(\vec{\omega} \cdot \mathbf{r})^{2}, \quad(\vec{\omega} \times \mathbf{r}) \cdot \hat{\mathbf{p}}=\vec{\omega} \cdot(\mathbf{r} \times \hat{\mathbf{p}})
$$

allows us to represent the kinetic energy operator (129) as

$$
\hat{K}_{\omega}=\hat{K}+\vec{\omega} \cdot \hat{\mathbf{L}}+\frac{m}{2} \int \hat{\psi}(\mathbf{r})\left[\omega^{2} r^{2}-(\vec{\omega} \cdot \mathbf{r})^{2}\right] \hat{\psi}(\mathbf{r}) d \mathbf{r}
$$

Thus, the energy Hamiltonian (128), in the laboratory frame, takes the form

$$
\hat{H}_{\omega}=\hat{H}+\vec{\omega} \cdot \hat{\mathbf{L}}+\frac{m}{2} \int \hat{\psi}(\mathbf{r})\left[\omega^{2} r^{2}-(\vec{\omega} \cdot \mathbf{r})^{2}\right] \hat{\psi}(\mathbf{r}) d \mathbf{r}
$$

Rotating systems are characterized by the inertia tensor

$$
\hat{I}_{\alpha \beta} \equiv \frac{\partial \hat{L}_{\omega}^{\alpha}}{\partial \omega_{\beta}}
$$

that, in view of Eq. (125), reads as

$$
\hat{I}_{\alpha \beta}=m \int \hat{\psi}^{\dagger}(\mathbf{r})\left(r^{2} \delta_{\alpha \beta}-r_{\alpha} r_{\beta}\right) \hat{\psi}(\mathbf{r}) d \mathbf{r}
$$

If one chooses the axis $\mathrm{z}$ in the direction of the angular velocity, so that

$$
\vec{\omega}=\omega \mathbf{e}_{z},
$$

then the angular momentum (125) is given by the expression

$$
\hat{L}_{\omega}^{z}=\hat{L}_{z}+\omega \hat{I}_{z z}
$$

with the inertia tensor

$$
\hat{I}_{z z}=m \int \hat{\psi}^{\dagger}(\mathbf{r})\left(x^{2}+y^{2}\right) \hat{\psi}(\mathbf{r}) d \mathbf{r}
$$

where the relation $r^{2}-z^{2}=x^{2}+y^{2}$ is used. The energy Hamiltonian (128), characterizing the system energy in the laboratory frame, can be represented as

$$
\hat{H}_{\omega}=\hat{H}+\omega \hat{L}_{z}+\frac{\omega^{2}}{2} \hat{I}_{z z}
$$

with $\hat{H}$ from Eq. (126).

The generalized superfluid fraction is defined as

$$
n_{s}(\omega) \equiv \frac{\frac{\partial}{\partial \omega}\left\langle\hat{L}_{\omega}^{z}\right\rangle_{\omega}}{\left\langle\frac{\partial}{\partial \omega} \hat{L}_{\omega}^{z}\right\rangle_{\omega}}
$$


For an equilibrium system, we can again employ the formulas of differentiation over parameters [76], leading to the derivative

$$
\frac{\partial}{\partial \omega}\left\langle\hat{L}_{\omega}^{z}\right\rangle_{\omega}=\left\langle\frac{\partial}{\partial \omega} \hat{L}_{\omega}^{z}\right\rangle_{\omega}-\beta \operatorname{cov}\left(\hat{L}_{\omega}^{z}, \frac{\partial \hat{H}_{\omega}}{\partial \omega}\right) .
$$

Substituting here

$$
\frac{\partial \hat{L}_{\omega}^{z}}{\partial \omega}=\hat{I}_{z z}, \quad \frac{\partial \hat{H}_{\omega}}{\partial \omega}=\hat{L}_{\omega}^{z}
$$

we come to the expression

$$
n_{s}(\omega)=1-\frac{\Delta^{2}\left(\hat{L}_{\omega}^{z}\right)}{T\left\langle\hat{I}_{z z}\right\rangle_{\omega}}
$$

Considering the superfluid fraction in the nonrotating limit,

$$
n_{s} \equiv \lim _{\omega \rightarrow 0} n_{s}(\omega)
$$

and using the notation

$$
I_{z z} \equiv \lim _{\omega \rightarrow 0}\left\langle\hat{I}_{z z}\right\rangle_{\omega}=m \int\left(x^{2}+y^{2}\right) \rho(\mathbf{r}) d \mathbf{r}
$$

we obtain the superfluid fraction in the form

$$
n_{s}=1-\frac{\Delta^{2}\left(\hat{L}_{z}\right)}{T I_{z z}} .
$$

The dispersion of $\hat{L}_{z}$ is calculated with the Hamiltonian for a nonrotating system.

Introducing the notation

$$
I_{e f f} \equiv \beta \Delta^{2}\left(\hat{L}_{z}\right)
$$

allows us to represent the superfluid fraction (143) as

$$
n_{s}=1-\frac{I_{e f f}}{I_{z z}}
$$

For a nonrotating system, one has

$$
\Delta^{2}\left(\hat{L}_{z}\right)=\left\langle\hat{L}_{z}^{2}\right\rangle \quad\left(\left\langle\hat{L}_{z}\right\rangle=0\right)
$$

Hence $I_{\text {eff }}=\beta<\hat{L}_{z}^{2}>$.

\subsection{Equivalence of Definitions}

The definitions of the superfluid fraction, considered in Sec. 5.1 and in Sec. 5.2, are equivalent with each other. To show this, one can take a cylindrical annulus of radius $R$, width $\delta$, and length $L$, such that $\delta \ll R$. The volume of this annulus is $V \simeq 2 \pi R L \delta$. Then the classical inertia tensor (142) is $I_{z z} \simeq m N R^{2}$. The angular momentum (122) can be written as

$$
\hat{L}_{z}=\int \hat{\psi}^{\dagger}(\mathbf{r})\left(-i \frac{\partial}{\partial \varphi}\right) \hat{\psi}(\mathbf{r}) d \mathbf{r}
$$


where $\varphi$ is the angle of the cylindrical system of coordinates.

For the annulus of large radius $R$, making the round along the annulus circumference, one has the path element $\delta l=R \delta \varphi$. Therefore the angular momentum (146) can be represented as

$$
\hat{L}_{z}=R \hat{P}_{l},
$$

being proportional to the momentum

$$
\hat{P}_{l} \equiv \int \hat{\psi}^{\dagger}(\mathbf{r})\left(-i \frac{\partial}{\partial l}\right) \hat{\psi}(\mathbf{r}) d \mathbf{r} .
$$

Then the superfluid fraction (143) becomes

$$
n_{s}=1-\frac{\Delta^{2}\left(\hat{P}_{l}\right)}{m N T} .
$$

The same formula follows from the consideration of Sec. 5.1, if one takes the velocity boost along the annulus circumference.

\subsection{Local Superfluidity}

In some cases, it is important to know the spatial distribution of the superfluid fraction that would be given by the spatial dependence $n_{s}(\mathbf{r})$. This can be necessary, when one considers equilibrium nonuniform systems or systems in local equilibrium $[79,80]$.

To describe local superfluidity, we can consider the momentum density

$$
\hat{\mathbf{P}}(\mathbf{r}) \equiv \hat{\psi}^{\dagger}(\mathbf{r}) \hat{\mathbf{p}} \hat{\psi}(\mathbf{r}) .
$$

Following Sec. 5.1, we introduce a velocity boost, which leads to the momentum density

$$
\hat{\mathbf{P}}_{v}(\mathbf{r}) \equiv \hat{\psi}^{\dagger}(\mathbf{r})(\hat{\mathbf{p}}+m \mathbf{v}) \hat{\psi}(\mathbf{r})
$$

in the laboratory frame. The local superfluid fraction is defined as

$$
n_{s}(\mathbf{r}) \equiv \lim _{v \rightarrow 0} \frac{\frac{\partial}{\partial \mathbf{v}} \cdot\left\langle\hat{\mathbf{P}}_{v}(\mathbf{r})\right\rangle_{v}}{\left\langle\frac{\partial}{\partial \mathbf{v}} \cdot \hat{\mathbf{P}}_{v}(\mathbf{r})\right\rangle_{v}}
$$

Because of form (151), one has

$$
\frac{\partial}{\partial \mathbf{v}} \cdot \hat{\mathbf{P}}_{v}(\mathbf{r})=3 m \hat{\psi}^{\dagger}(\mathbf{r}) \hat{\psi}(\mathbf{r}) .
$$

Then the local superfluid fraction (152) reduces to

$$
n_{s}(\mathbf{r})=1-\frac{\operatorname{cov}(\hat{\mathbf{P}}(\mathbf{r}), \hat{\mathbf{P}})}{3 m \rho(\mathbf{r}) T} .
$$

Owing to the relation

$$
\rho_{s}(\mathbf{r})=n_{s}(\mathbf{r}) \rho(\mathbf{r}),
$$

we get the local superfluid density

$$
\rho_{s}(\mathbf{r})=\rho(\mathbf{r})-\frac{\operatorname{cov}(\hat{\mathbf{P}}(\mathbf{r}), \hat{\mathbf{P}})}{3 m T} .
$$

Integrating the above equation over $\mathbf{r}$ and considering the average fraction

$$
n_{s}=\frac{1}{N} \int \rho_{s}(\mathbf{r}) d \mathbf{r}
$$

would bring us back to formula (117). 


\subsection{Superfluidity and Condensation}

Usually, Bose-Einstein condensation is accompanied by superfluidity. However, there is no straightforward relation between these phenomena and the related fractions $[3,12]$. Thus, in two-dimensional systems at finite temperature, there is no Bose condensation, but there can exist superfluidity. And in spatially random systems, there can happen local Bose condensation without superfluidity.

The relation between Bose condensation and superfluidity depends on the type of the effective particle spectrum and system dimensionality. To illustrate this, let us consider a $d$-dimensional Bose gas with an effective particle spectrum

$$
\omega_{k}=A k^{n}-\mu
$$

where $A$ and $n$ are positive parameters and $\mathbf{k}$ is $d$-dimensional momentum. For the $d$-dimensional case, the superfluid fraction (117) takes the form

$$
n_{s}=1-\frac{\left\langle\hat{\mathbf{P}}^{2}\right\rangle}{N m T d}
$$

The integration over the $d$-dimensional momenta involves the relation

$$
\frac{d \mathbf{k}}{(2 \pi)^{d}} \rightarrow \frac{2 k^{d-1} d k}{(4 \pi)^{d / 2} \Gamma(d / 2)},
$$

in which $\Gamma(x)$ is the gamma function.

For the condensation temperature, we find

$$
T_{c}=A\left[\frac{(4 \pi)^{d / 2} \Gamma(d / 2) n \rho}{2 \Gamma(d / n) \zeta(d / n)}\right]^{n / d},
$$

where $\zeta(x)$ is the Riemann zeta function. The latter, can be represented in several forms:

$$
\zeta(x)=\sum_{j=1}^{\infty} \frac{1}{j^{x}}=\frac{1}{\Gamma(x)} \int_{0}^{\infty} \frac{t^{x-1}}{e^{t}-1} d t
$$

when $\operatorname{Re} x>1$, and

$$
\zeta(x)=\frac{1}{\left(1-2^{1-x}\right) \Gamma(x)} \int_{0}^{\infty} \frac{u^{x-1}}{e^{u}+1} d u
$$

if $\operatorname{Re} x>0$.

Taking into account that $\Gamma(x)>0$ for $x>0$ and $\zeta(x)<0$ in the interval $0<x<1$ tells us that there is no condensation for $d<n$. When $d=n$, then $T_{c}=0$. And $T_{c}>0$ for $d>n$.

For $d>n$, the condensate fraction below $T_{c}$ is given by the expression

$$
n_{0}=1-\left(\frac{T}{T_{c}}\right)^{d / n} \quad\left(T \leq T_{c}\right)
$$

while the superfluid fraction, under $\mu=0$, is

$$
n_{s}=1-B \zeta\left(\frac{d+2-n}{n}\right) T^{(d+2-n) / n}
$$


where

$$
B \equiv \frac{2(d+2-n) \Gamma\left(\frac{d+2-n}{n}\right)}{(4 \pi)^{d / 2} \Gamma\left(\frac{d}{2}\right) A^{(d+2) / n} m \rho n^{2} d} .
$$

If there is no condensate, then $\mu$ is defined by the equation

$$
\rho=\frac{2 \Gamma(d / n) g_{d / n}(z) T^{d / n}}{(4 \pi)^{d / 2} \Gamma(d / 2) n A^{d / n}}
$$

in which $z \equiv e^{\beta \mu}$ is fugacity and

$$
g_{n}(z) \equiv \frac{1}{\Gamma(n)} \int_{0}^{\infty} \frac{z u^{n-1}}{e^{u}-z} d u
$$

is the Bose function. The superfluid fraction, in the absence of condensate, is

$$
n_{s}=1-B g_{(d+2-n) / n}(z) T^{(d+2-n) / n} .
$$

Generally speaking, Bose-Einstein condensation is neither necessary nor sufficient for superfluidity. These phenomena are connected with different system features. Bose condensation implies the appearance of coherence in the system, while superfluidity is related to the presence of sufficiently strong pair correlations. Thus, there can occur four possibilities, depending on the values of the condensate and superfluid fractions:

(i) incoherent normal fluid

$$
n_{0}=0, \quad n_{s}=0
$$

(ii) coherent normal fluid

$$
n_{0}>0, \quad n_{s}=0
$$

(iii) incoherent superfluid

$$
n_{0}=0, \quad n_{s}>0
$$

(iv) coherent superfluid

$$
n_{0}>0, \quad n_{s}>0 .
$$

In this classification, we do not take into account that the system can form a solid [12].

\section{Equilibrium Uniform Systems}

\subsection{Information Functional}

The definition of statistical averages involves the use of a statistical operator. The form of the latter, in the case of an equilibrium system, can be found from the principle of minimal information. This principle requires that, composing an information functional, one has to take into account all conditions and constraints that uniquely define the considered system [81]. Only then the corresponding statistical ensemble will be representative and will correctly describe the system. In the other case, if not all necessary constraints have been taken into account, so that the system is not uniquely described, the ensemble is not representative and cannot correctly characterize the system. In such a case, one confronts different problems, for instance, the occurrence of thermodynamic instability or nonequivalence of ensembles. However, all those problems are caused by the use of nonrepresentative ensembles and have nothing to do with 
physics. A detailed discussion of these problems can be found in Ref. [63]. The construction of representative ensembles for Bose-condensed systems is given in Refs. [64,71].

A statistical operator $\hat{\rho}$ of an equilibrium system should be the minimizer of the Shannon information $\hat{\rho} \ln \hat{\rho}$, under given statistical conditions. The first evident condition is the normalization

$$
\langle\hat{1}\rangle \equiv \operatorname{Tr} \hat{\rho}=1
$$

with $\hat{1}$ being the unity operator. Then, one defines the internal energy $E$ through the average

$$
\langle\hat{H}\rangle \equiv \operatorname{Tr} \hat{\rho} \hat{H}=E .
$$

The normalization condition (52) for the condensate function can also be presented in the standard form of a statistical condition as

$$
\left\langle\hat{N}_{0}\right\rangle \equiv \operatorname{Tr} \hat{\rho} \hat{N}_{0}=N_{0}
$$

where $\hat{N}_{0} \equiv N_{0} \hat{1}$. Normalization (53), for the number of uncondensed particles, can be written as

$$
\left\langle\hat{N}_{1}\right\rangle \equiv \operatorname{Tr} \hat{\rho} \hat{N}_{1}=N_{1} .
$$

Finally, the conservation condition (57) reads as

$$
\langle\hat{\Lambda}\rangle \equiv \operatorname{Tr} \hat{\rho} \hat{\Lambda}=0 .
$$

Note that, in general, the conditional operators do not need to be necessarily commutative with the energy operator [80]. For instance, here the operator $\hat{N}_{0}$ commutes with $\hat{H}$, but $\hat{\Lambda}$ does not have to commute with the latter.

It is also worth stressing that the average quantities, involved in the statistical conditions, do not need to be directly prescribed, but they have to be uniquely defined by fixing other thermodynamic parameters. Thus, internal energy is not prescribed directly in either canonical or grand canonical ensembles, but it is uniquely defined through the fixed temperature, the number of particles in the system, and volume. Similarly, the number of condensed particles may be not directly given, but it is uniquely defined, and can be measured, by fixing other thermodynamic parameters, temperature, total number of particles, and volume. For confined systems, instead of volume, the external potential is given.

The information functional, under the above conditions, takes the form

$$
\begin{gathered}
I[\hat{\rho}]=\operatorname{Tr} \hat{\rho} \ln \hat{\rho}+\lambda_{0}(\operatorname{Tr} \hat{\rho}-1)+\beta(\operatorname{Tr} \hat{\rho} \hat{H}-E)- \\
-\beta \mu_{0}\left(\operatorname{Tr} \hat{\rho} \hat{N}_{0}-N_{0}\right)-\beta \mu_{1}\left(\operatorname{Tr} \hat{\rho} \hat{N}_{1}-N_{1}\right)-\beta \operatorname{Tr} \hat{\rho} \hat{\Lambda}
\end{gathered}
$$

in which the corresponding Lagrange multipliers are introduced. Minimizing this functional with respect to $\hat{\rho}$ yields the statistical operator

$$
\hat{\rho}=\frac{e^{-\beta H}}{\operatorname{Tr} e^{-\beta H}}
$$

with the same grand Hamiltonian (58). 


\subsection{Momentum Representation}

For a uniform system, it is convenient to pass to the momentum representation by means of the Fourier transformation with plane waves. This is because the plane waves are the natural orbitals for a uniform system, which implies that they are the eigenfunctions of the density matrix in the sense of eigenproblem (5).

The field operator of uncondensed particles transforms as

$$
\psi_{1}(\mathbf{r})=\frac{1}{\sqrt{V}} \sum_{k \neq 0} a_{k} e^{i \mathbf{k} \cdot \mathbf{r}}, \quad a_{k}=\frac{1}{\sqrt{V}} \int \psi_{1}(\mathbf{r}) e^{-i \mathbf{k} \cdot \mathbf{r}} d \mathbf{r}
$$

We assume that the pair interaction potential is Fourier transformable,

$$
\Phi(\mathbf{r})=\frac{1}{V} \sum_{k} \Phi_{k} e^{i \mathbf{k} \cdot \mathbf{r}}, \quad \Phi_{k}=\int \Phi(\mathbf{r}) e^{-i \mathbf{k} \cdot \mathbf{r}} d \mathbf{r} .
$$

The condensate function $\eta(\mathbf{r})$ for a uniform system, is a constant $\eta$, such that

$$
\rho_{0}(\mathbf{r})=|\eta|^{2}=\rho_{0}
$$

These transformations are substituted into the grand Hamiltonian (67). Then the zero-order term (68) becomes

$$
H^{(0)}=\left(\frac{1}{2} \rho_{0} \Phi_{0}-\mu_{0}\right) N_{0}
$$

The first-order term $H_{1}$ is automatically zero, as in Eq. (69). The second-order term (70) reads as

$$
\begin{aligned}
H^{(2)}= & \sum_{k \neq 0}\left[\frac{k^{2}}{2 m}+\rho_{0}\left(\Phi_{0}+\Phi_{k}\right)-\mu_{1}\right] a_{k}^{\dagger} a_{k}+ \\
& +\frac{1}{2} \sum_{k \neq 0} \rho_{0} \Phi_{k}\left(a_{k}^{\dagger} a_{-k}^{\dagger}+a_{-k} a_{k}\right) .
\end{aligned}
$$

The third-order term $(71)$ is

$$
H^{(3)}=\sqrt{\frac{\rho_{0}}{V}} \sum_{k p}^{\prime} \Phi_{p}\left(a_{k}^{\dagger} a_{k+p} a_{-p}+a_{-p}^{\dagger} a_{k+p}^{\dagger} a_{k}\right),
$$

where in the sum

$$
\mathbf{k} \neq 0, \quad \mathbf{p} \neq 0, \quad \mathbf{k}+\mathbf{p} \neq 0 .
$$

The fourth-order term (72) takes the form

$$
H^{(4)}=\frac{1}{2 V} \sum_{q} \sum_{k p}^{\prime} \Phi_{q} a_{k}^{\dagger} a_{p}^{\dagger} a_{p+q} a_{k-q},
$$

where

$$
\mathbf{k} \neq 0, \quad \mathbf{p} \neq 0, \quad \mathbf{p}+\mathbf{q} \neq 0, \quad \mathbf{k}-\mathbf{q} \neq 0
$$




\subsection{Condensate Function}

In the case of an equilibrium system, the condensate function does not depend on time,

$$
\frac{\partial}{\partial t} \eta(\mathbf{r}, t)=0
$$

Therefore, Eq. (95) reduces to the eigenvalue problem

$$
\begin{gathered}
{\left[-\frac{\nabla^{2}}{2 m}+U(\mathbf{r})\right] \eta(\mathbf{r})+} \\
+\int \Phi\left(\mathbf{r}-\mathbf{r}^{\prime}\right)\left[\rho\left(\mathbf{r}^{\prime}\right) \eta(\mathbf{r})+\rho_{1}\left(\mathbf{r}, \mathbf{r}^{\prime}\right) \eta\left(\mathbf{r}^{\prime}\right)+\sigma_{1}\left(\mathbf{r}, \mathbf{r}^{\prime}\right) \eta^{*}\left(\mathbf{r}^{\prime}\right)+\xi\left(\mathbf{r}, \mathbf{r}^{\prime}\right)\right] d \mathbf{r}^{\prime}=\mu_{0} \eta(\mathbf{r}) .
\end{gathered}
$$

A uniform system presupposes the absence of a nonuniform external potential. Hence, one can set $U=0$. The average densities $\rho_{0}$ and $\rho_{1}$ are constant. The total particle density is

$$
\rho=\rho(\mathbf{r})=\rho_{0}+\rho_{1} .
$$

Then Eq. (181) gives

$$
\mu_{0}=\rho \Phi_{0}+\int \Phi(\mathbf{r})\left[\rho_{1}(\mathbf{r}, 0)+\sigma_{1}(\mathbf{r}, 0)+\frac{\xi(\mathbf{r}, 0)}{\sqrt{\rho_{0}}}\right] d \mathbf{r}
$$

The normal density matrix is written as

$$
\rho_{1}\left(\mathbf{r}, \mathbf{r}^{\prime}\right)=\frac{1}{V} \sum_{k \neq 0} n_{k} e^{i \mathbf{k} \cdot\left(\mathbf{r}-\mathbf{r}^{\prime}\right)}
$$

where

$$
n_{k} \equiv\left\langle a_{k}^{\dagger} a_{k}\right\rangle
$$

And the anomalous average

$$
\sigma_{1}\left(\mathbf{r}, \mathbf{r}^{\prime}\right)=\frac{1}{V} \sum_{k \neq 0} \sigma_{k} e^{i \mathbf{k} \cdot\left(\mathbf{r}-\mathbf{r}^{\prime}\right)}
$$

is expressed through

$$
\sigma_{k} \equiv\left\langle a_{k} a_{-k}\right\rangle \text {. }
$$

The triple anomalous correlator (94) can be represented as

$$
\xi\left(\mathbf{r}, \mathbf{r}^{\prime}\right)=\frac{1}{V} \sum_{k \neq 0} \xi_{k} e^{i \mathbf{k} \cdot\left(\mathbf{r}-\mathbf{r}^{\prime}\right)},
$$

with

$$
\xi_{k}=\frac{1}{\sqrt{V}} \sum_{p \neq 0}\left\langle a_{k} a_{p} a_{-k-p}\right\rangle .
$$

The diagonal element of Eq. (184) gives the density of uncondensed particles

$$
\rho_{1}=\rho_{1}(\mathbf{r}, \mathbf{r})=\frac{1}{V} \sum_{k \neq 0} n_{k}
$$


The diagonal element of the anomalous average (186) is

$$
\sigma_{1}=\sigma_{1}(\mathbf{r}, \mathbf{r})=\frac{1}{V} \sum_{k \neq 0} \sigma_{k}
$$

And the triple correlator (188) leads to

$$
\xi=\xi(\mathbf{r}, \mathbf{r})=\frac{1}{V} \sum_{k \neq 0} \xi_{k} .
$$

The condensate chemical potential (183) can be rewritten in the form

$$
\mu_{0}=\rho \Phi_{0}+\frac{1}{V} \sum_{k \neq 0}\left(n_{k}+\sigma_{k}+\frac{\xi_{k}}{\sqrt{\rho_{0}}}\right) \Phi_{k} .
$$

\subsection{Green Functions}

There are several types of Green functions. Here, we shall deal with the causal Green functions $[81,82]$ that are called propagators. The set $\left\{\mathbf{r}_{j}, t_{j}\right\}$ of the spatial variable $\mathbf{r}_{j}$ and time $t_{j}$ will be denoted, for brevity, just as $j$. If there are other internal variables, they can also be included in the notation $j$.

For a Bose-condensed system, one considers four types of Green functions:

$$
\begin{array}{ll}
G_{11}(12)=-i\left\langle\hat{T} \psi_{1}(1) \psi_{1}^{\dagger}(2)\right\rangle, & G_{12}(12)=-i\left\langle\hat{T} \psi_{1}(1) \psi_{1}(2)\right\rangle, \\
G_{21}(12)=-i\left\langle\hat{T} \psi_{1}^{\dagger}(1) \psi_{1}^{\dagger}(2)\right\rangle, & G_{22}(12)=-i\left\langle\hat{T} \psi_{1}^{\dagger}(1) \psi_{1}(2)\right\rangle,
\end{array}
$$

in which $\hat{T}$ is chronological operator. It is convenient [83] to introduce the retarded interaction

$$
\Phi(12) \equiv \Phi\left(\mathbf{r}_{1}-\mathbf{r}_{2}\right) \delta\left(t_{1}-t_{2}+0\right) .
$$

Also, one defines the inverse propagators

$$
\begin{gathered}
G_{11}^{-1}(12)=\left[i \frac{\partial}{\partial t_{1}}+\frac{\nabla_{1}^{2}}{2 m}-U(1)+\mu_{1}\right] \delta(12)-\Sigma_{11}(12), \\
G_{12}^{-1}(12)=-\Sigma_{12}(12), \quad G_{21}^{-1}(12)=-\Sigma_{21}(12), \\
G_{22}^{-1}(12)=\left[-i \frac{\partial}{\partial t_{1}}+\frac{\nabla_{1}^{2}}{2 m}-U(1)+\mu_{1}\right] \delta(12)-\Sigma_{22}(12),
\end{gathered}
$$

where $\Sigma_{\alpha \beta}(12)$ is self-energy. Using these, one can write the equations of motion in the matrix form

$$
\begin{array}{ll}
G_{11}^{-1} G_{11}+G_{12}^{-1} G_{21}=\hat{1}, & G_{11}^{-1} G_{12}+G_{12}^{-1} G_{22}=0, \\
G_{21}^{-1} G_{11}+G_{22}^{-1} G_{21}=0, & G_{21}^{-1} G_{12}+G_{22}^{-1} G_{22}=\hat{1} .
\end{array}
$$

For a uniform system, when $U=0$, one passes to the Fourier transforms of the Green functions $G_{\alpha \beta}(\mathbf{k}, \omega)$, inverse propagators $G_{\alpha \beta}^{-1}(\mathbf{k}, \omega)$, and self-energies $\Sigma_{\alpha \beta}(\mathbf{k}, \omega)$. The inverse propagators (196) transform into

$$
G_{11}^{-1}(\mathbf{k}, \omega)=\omega-\frac{k^{2}}{2 m}+\mu_{1}-\Sigma_{11}(\mathbf{k}, \omega), \quad G_{12}^{-1}(\mathbf{k}, \omega)=-\Sigma_{12}(\mathbf{k}, \omega),
$$




$$
G_{21}^{-1}(\mathbf{k}, \omega)=-\Sigma_{21}(\mathbf{k}, \omega), \quad G_{22}^{-1}(\mathbf{k}, \omega)=-\omega-\frac{k^{2}}{2 m}+\mu_{1}-\Sigma_{22}(\mathbf{k}, \omega) .
$$

The Green functions enjoy the properties

$$
\begin{gathered}
G_{\alpha \beta}(-\mathbf{k}, \omega)=G_{\alpha \beta}(\mathbf{k}, \omega), \quad G_{11}(\mathbf{k},-\omega)=G_{22}(\mathbf{k}, \omega) \\
G_{12}(\mathbf{k},-\omega)=G_{21}(\mathbf{k}, \omega)=G_{12}(\mathbf{k}, \omega) .
\end{gathered}
$$

And the self-energies also share the same properties

$$
\begin{gathered}
\Sigma_{\alpha \beta}(-\mathbf{k}, \omega)=\Sigma_{\alpha \beta}(\mathbf{k}, \omega), \quad \Sigma_{11}(\mathbf{k},-\omega)=\Sigma_{22}(\mathbf{k}, \omega), \\
\Sigma_{12}(\mathbf{k},-\omega)=\Sigma_{21}(\mathbf{k}, \omega)=\Sigma_{12}(\mathbf{k}, \omega) .
\end{gathered}
$$

Equations (197) yield

$$
G_{11}(\mathbf{k}, \omega)=\frac{\omega+k^{2} / 2 m-\mu_{1}+\Sigma_{11}(\mathbf{k}, \omega)}{D(\mathbf{k}, \omega)}, \quad G_{12}(\mathbf{k}, \omega)=-\frac{\Sigma_{12}(\mathbf{k}, \omega)}{D(\mathbf{k}, \omega)}
$$

with the denominator

$$
D(\mathbf{k}, \omega)=\Sigma_{12}^{2}(\mathbf{k}, \omega)-G_{11}^{-1}(\mathbf{k}, \omega) G_{22}^{-1}(\mathbf{k}, \omega) .
$$

\subsection{Hugenholtz-Pines Relation}

Hugenholtz and Pines [37], using perturbation theory at zero temperature, found the relation

$$
\mu_{1}=\Sigma_{11}(0,0)-\Sigma_{12}(0,0) .
$$

The most general proof of this relation, for any temperature, was given by Bogolubov [16]. He proved the theorem, according to which

$$
\left|G_{11}(\mathbf{k}, 0)\right| \geq \frac{m n_{0}}{2 k^{2}}
$$

where $n_{0}$ is the condensate fraction, and

$$
\left|G_{11}(\mathbf{k}, 0)-G_{12}(\mathbf{k}, 0)\right| \geq \frac{m n_{0}}{k^{2}} .
$$

¿From inequality (204), one has

$$
\lim _{k \rightarrow 0} \lim _{\omega \rightarrow 0} D(\mathbf{k}, \omega)=0 .
$$

And from inequality (205), it follows that

$$
\left|\frac{k^{2}}{2 m}-\mu_{1}+\Sigma_{11}(\mathbf{k}, 0)-\Sigma_{12}(\mathbf{k}, 0)\right| \leq \frac{k^{2}}{m n_{0}} .
$$

The latter inequality leads to the Hugenholtz-Pines relation (203).

It is important to stress that the expression for $\mu_{1}$, given by Eq. (203), is exact and, generally, it is different from the exact value of $\mu_{0}$ in Eq. (183). 
The Hugenholtz-Pines relation is equivalent to the fact that the particle spectrum is gapless, which follows from the following.

The spectrum $\varepsilon_{k}$ is given by the zeroes of the Green-function denominator:

$$
D\left(\mathbf{k}, \varepsilon_{k}\right)=0
$$

which gives the equation

$$
\varepsilon_{k}=\frac{1}{2}\left[\Sigma_{11}\left(\mathbf{k}, \varepsilon_{k}\right)-\Sigma_{22}\left(\mathbf{k}, \varepsilon_{k}\right)\right]+\sqrt{\omega_{k}^{2}-\Sigma_{12}^{2}\left(\mathbf{k}, \varepsilon_{k}\right)}
$$

where

$$
\omega_{k} \equiv \frac{k^{2}}{2 m}+\frac{1}{2}\left[\Sigma_{11}\left(\mathbf{k}, \varepsilon_{k}\right)+\Sigma_{22}\left(\mathbf{k}, \varepsilon_{k}\right)\right]-\mu_{1} .
$$

In view of condition (206), the limit

$$
\lim _{k \rightarrow 0} \varepsilon_{k}=0
$$

is valid, that is, the spectrum is gapless.

To find the long-wave spectrum behavior, keeping in mind that the spectrum is uniquely defined by Eq. (209), we can use the expansion

$$
\Sigma_{\alpha \beta}\left(k, \varepsilon_{k}\right) \simeq \Sigma_{\alpha \beta}(0,0)+\Sigma_{\alpha \beta}^{\prime} k^{2},
$$

in which $k \rightarrow 0$ and

$$
\Sigma_{\alpha \beta}^{\prime} \equiv \lim _{k \rightarrow 0} \frac{\partial}{\partial k^{2}} \Sigma_{\alpha \beta}\left(\mathbf{k}, \varepsilon_{k}\right) .
$$

Then, defining the sound velocity

$$
c \equiv \sqrt{\frac{1}{m^{*}} \Sigma_{12}(0,0)}
$$

and the effective mass

$$
m^{*} \equiv \frac{m}{1+m\left(\Sigma_{11}^{\prime}+\Sigma_{22}^{\prime}-2 \Sigma_{12}^{\prime}\right)},
$$

we get the acoustic spectrum

$$
\varepsilon_{k} \simeq c k \quad(k \rightarrow 0) .
$$

Equation (213), characterizing the general feature of the long-wave spectrum, has been obtained without approximations, assuming only the validity of expansion (212). Therefore, in a Bose-condensed system, the anomalous self-energy $\Sigma_{12}(0,0)$ must be nonzero in order to define a meaningful nonzero sound velocity. The zero sound velocity would mean the system instability. Since expression (213) involves no perturbation theory and no approximations, the condition

$$
\Sigma_{12}(0,0) \neq 0
$$

is general, as soon as expansion (212) is valid. 


\section{Hartree-Fock-Bogolubov Approximation}

\subsection{Nonuniform Matter}

To realize practical calculations, it is necessary to resort to some approximation. The Bogolubov approximation $[13,14]$ is valid for low temperatures and asymptotically weak interactions. The more general approximation, that would be valid for all temperatures and any interaction strength, is the Hartree-Fock-Bogolubov (HFB) approximation. Early works [35,36], employing this approximation, confronted the inconsistency problem discussed in Sec. 1, because of a gap in the particle spectrum. This happened as a result of the use of a nonrepresentative ensemble. Employing the representative ensemble of Sec. 4 yields no gap and no any other problems. The HFB approximation, applied in the frame of the self-consistent theory of Sec. 4, is gapless and conserving [63-71].

The HFB approximation simplifies the general Hamiltonian (67). For generality, we consider, first, the nonuniform case.

The third-order term (71) in the HFB approximation is zero. And in the fourth-order term (72), the HFB approximation gives

$$
\begin{gathered}
\psi_{1}^{\dagger}(\mathbf{r}) \psi_{1}^{\dagger}\left(\mathbf{r}^{\prime}\right) \psi_{1}\left(\mathbf{r}^{\prime}\right) \psi_{1}(\mathbf{r})=\rho_{1}(\mathbf{r}) \psi_{1}^{\dagger}\left(\mathbf{r}^{\prime}\right) \psi_{1}\left(\mathbf{r}^{\prime}\right)+\rho_{1}\left(\mathbf{r}^{\prime}\right) \psi_{1}^{\dagger}(\mathbf{r}) \psi_{1}(\mathbf{r})+\rho_{1}\left(\mathbf{r}^{\prime}, \mathbf{r}\right) \psi_{1}^{\dagger}\left(\mathbf{r}^{\prime}\right) \psi_{1}(\mathbf{r})+ \\
+\rho_{1}\left(\mathbf{r}, \mathbf{r}^{\prime}\right) \psi_{1}^{\dagger}(\mathbf{r}) \psi_{1}\left(\mathbf{r}^{\prime}\right)+\sigma_{1}\left(\mathbf{r}, \mathbf{r}^{\prime}\right) \psi_{1}^{\dagger}(\mathbf{r}) \psi_{1}\left(\mathbf{r}^{\prime}\right)+ \\
+\sigma_{1}^{*}\left(\mathbf{r}^{\prime}, \mathbf{r}\right) \psi_{1}^{\dagger}\left(\mathbf{r}^{\prime}\right) \psi_{1}(\mathbf{r})-\rho_{1}(\mathbf{r}) \rho_{1}\left(\mathbf{r}^{\prime}\right)-\left|\rho_{1}\left(\mathbf{r}, \mathbf{r}^{\prime}\right)\right|^{2}-\left|\sigma_{1}\left(\mathbf{r}, \mathbf{r}^{\prime}\right)\right|^{2}
\end{gathered}
$$

In what follows, it is convenient to use the notation for the total single-particle density matrix

$$
\rho\left(\mathbf{r}, \mathbf{r}^{\prime}\right) \equiv \eta(\mathbf{r}) \eta^{*}\left(\mathbf{r}^{\prime}\right)+\rho_{1}\left(\mathbf{r}, \mathbf{r}^{\prime}\right)
$$

and for the total anomalous average

$$
\sigma\left(\mathbf{r}, \mathbf{r}^{\prime}\right) \equiv \eta(\mathbf{r}) \eta\left(\mathbf{r}^{\prime}\right)+\sigma_{1}\left(\mathbf{r}, \mathbf{r}^{\prime}\right)
$$

These equations reduce the grand Hamiltonian (67) to the HFB form

$$
\begin{aligned}
& H_{H F B}=E_{H F B}+\int \psi_{1}^{\dagger}(\mathbf{r})\left(-\frac{\nabla^{2}}{2 m}+U-\mu_{1}\right) \psi_{1}(\mathbf{r}) d \mathbf{r}+ \\
& +\int \Phi\left(\mathbf{r}-\mathbf{r}^{\prime}\right)\left[\rho\left(\mathbf{r}^{\prime}\right) \psi_{1}^{\dagger}(\mathbf{r}) \psi_{1}(\mathbf{r})+\rho\left(\mathbf{r}^{\prime}, \mathbf{r}\right) \psi_{1}^{\dagger}\left(\mathbf{r}^{\prime}\right) \psi_{1}(\mathbf{r})+\right. \\
& \left.+\frac{1}{2} \sigma\left(\mathbf{r}, \mathbf{r}^{\prime}\right) \psi_{1}^{\dagger}\left(\mathbf{r}^{\prime}\right) \psi_{1}^{\dagger}(\mathbf{r})+\frac{1}{2} \sigma^{*}\left(\mathbf{r}, \mathbf{r}^{\prime}\right) \psi_{1}\left(\mathbf{r}^{\prime}\right) \psi_{1}(\mathbf{r})\right] d \mathbf{r} d \mathbf{r}^{\prime}
\end{aligned}
$$

in which the nonoperator term is

$$
E_{H F B}=H^{(0)}-\frac{1}{2} \int \Phi\left(\mathbf{r}-\mathbf{r}^{\prime}\right)\left[\rho_{1}(\mathbf{r}) \rho_{1}\left(\mathbf{r}^{\prime}\right)+\left|\rho_{1}\left(\mathbf{r}, \mathbf{r}^{\prime}\right)\right|^{2}+\left|\sigma_{1}\left(\mathbf{r}, \mathbf{r}^{\prime}\right)\right|^{2}\right] d \mathbf{r} d \mathbf{r}^{\prime}
$$

The condensate-function equation (95) becomes

$$
i \frac{\partial}{\partial t} \eta(\mathbf{r}, t)=\left(-\frac{\nabla^{2}}{2 m}+U-\mu_{0}\right) \eta(\mathbf{r})+
$$




$$
+\int \Phi\left(\mathbf{r}-\mathbf{r}^{\prime}\right)\left[\rho\left(\mathbf{r}^{\prime}\right) \eta(\mathbf{r})+\rho_{1}\left(\mathbf{r}, \mathbf{r}^{\prime}\right) \eta\left(\mathbf{r}^{\prime}\right)+\sigma_{1}\left(\mathbf{r}, \mathbf{r}^{\prime}\right) \eta^{*}\left(\mathbf{r}^{\prime}\right)\right] d \mathbf{r}^{\prime}
$$

And the equation of motion (85) for the operator of uncondensed particles now reads as

$$
\begin{gathered}
i \frac{\partial}{\partial t} \psi_{1}(\mathbf{r}, t)=\left(-\frac{\nabla^{2}}{2 m}+U-\mu_{1}\right) \psi_{1}(\mathbf{r})+ \\
+\int \Phi\left(\mathbf{r}-\mathbf{r}^{\prime}\right)\left[\rho\left(\mathbf{r}^{\prime}\right) \psi_{1}(\mathbf{r})+\rho\left(\mathbf{r}, \mathbf{r}^{\prime}\right) \psi_{1}\left(\mathbf{r}^{\prime}\right)+\sigma\left(\mathbf{r}, \mathbf{r}^{\prime}\right) \psi_{1}^{\dagger}\left(\mathbf{r}^{\prime}\right)\right] d \mathbf{r}^{\prime} .
\end{gathered}
$$

In the case of an equilibrium system, Eq. (221) reduces to the eigenproblem

$$
\begin{gathered}
\left(-\frac{\nabla^{2}}{2 m}+U\right) \eta(\mathbf{r})+ \\
+\int \Phi\left(\mathbf{r}-\mathbf{r}^{\prime}\right)\left[\rho\left(\mathbf{r}^{\prime}\right) \eta(\mathbf{r})+\rho_{1}\left(\mathbf{r}, \mathbf{r}^{\prime}\right) \eta\left(\mathbf{r}^{\prime}\right)+\sigma_{1}\left(\mathbf{r}, \mathbf{r}^{\prime}\right) \eta^{*}\left(\mathbf{r}^{\prime}\right)\right] d \mathbf{r}^{\prime}=\mu_{0} \eta(\mathbf{r})
\end{gathered}
$$

defining the condensate function and the the condensate chemical potential

$$
\begin{gathered}
\mu_{0}=\frac{1}{N_{0}} \int \eta^{*}(\mathbf{r})\left[-\frac{\nabla^{2}}{2 m}+U(\mathbf{r})\right] \eta(\mathbf{r}) d \mathbf{r}+ \\
+\frac{1}{N_{0}} \int \Phi\left(\mathbf{r}-\mathbf{r}^{\prime}\right)\left[\rho_{0}(\mathbf{r}) \rho\left(\mathbf{r}^{\prime}\right)+\rho_{1}\left(\mathbf{r}, \mathbf{r}^{\prime}\right) \eta^{*}(\mathbf{r}) \eta\left(\mathbf{r}^{\prime}\right)+\sigma_{1}\left(\mathbf{r}, \mathbf{r}^{\prime}\right) \eta^{*}(\mathbf{r}) \eta^{*}\left(\mathbf{r}^{\prime}\right)\right] d \mathbf{r} d \mathbf{r}^{\prime}
\end{gathered}
$$

\subsection{Bogolubov Transformations}

The HFB Hamiltonian (219) is a quadratic form with respect to the operators $\psi_{1}$. As any quadratic form, it can be diagonalized by means of the Bogolubov canonical transformations, whose general properties are described in detail in the book [84]. In the present case, the Bogolubov transformations read as

$$
\psi_{1}(\mathbf{r})=\sum_{k}\left[u_{k}(\mathbf{r}) b_{k}+v_{k}^{*}(\mathbf{r}) b_{k}^{\dagger}\right], \quad b_{k}=\int\left[u_{k}^{*}(\mathbf{r}) \psi_{1}(\mathbf{r})-v_{k}^{*}(\mathbf{r}) \psi_{1}^{\dagger}(\mathbf{r})\right] d \mathbf{r}
$$

Since $\psi_{1}$ is a Bose operator, it should be:

$$
\begin{gathered}
\sum_{k}\left[u_{k}(\mathbf{r}) u_{k}^{*}\left(\mathbf{r}^{\prime}\right)-v_{k}^{*}(\mathbf{r}) v_{k}\left(\mathbf{r}^{\prime}\right)\right]=\delta\left(\mathbf{r}-\mathbf{r}^{\prime}\right), \\
\sum_{k}\left[u_{k}(\mathbf{r}) v_{k}^{*}\left(\mathbf{r}^{\prime}\right)-v_{k}^{*}(\mathbf{r}) u_{k}\left(\mathbf{r}^{\prime}\right)\right]=0 .
\end{gathered}
$$

And, the condition that $b_{k}$ is also a Bose operator leads to the relations

$$
\int\left[u_{k}^{*}(\mathbf{r}) u_{p}(\mathbf{r})-v_{k}^{*}(\mathbf{r}) v_{p}(\mathbf{r})\right] d \mathbf{r}=\delta_{k p}, \quad \int\left[u_{k}(\mathbf{r}) v_{p}(\mathbf{r})-v_{k}(\mathbf{r}) u_{p}(\mathbf{r})\right] d \mathbf{r}=0 .
$$

The coefficient functions $u_{k}$ and $v_{k}$ are to be defined by the requirement of the Hamiltonian diagonalization, under conditions (226) and (227). 
Let us introduce the notations

$$
\begin{gathered}
\omega\left(\mathbf{r}, \mathbf{r}^{\prime}\right) \equiv\left[-\frac{\nabla^{2}}{2 m}+U(\mathbf{r})-\mu_{1}+\int \Phi\left(\mathbf{r}-\mathbf{r}^{\prime}\right) \rho\left(\mathbf{r}^{\prime}\right) d \mathbf{r}^{\prime}\right] \delta\left(\mathbf{r}-\mathbf{r}^{\prime}\right)+ \\
+\Phi\left(\mathbf{r}-\mathbf{r}^{\prime}\right) \rho\left(\mathbf{r}, \mathbf{r}^{\prime}\right)
\end{gathered}
$$

and

$$
\Delta\left(\mathbf{r}, \mathbf{r}^{\prime}\right) \equiv \Phi\left(\mathbf{r}-\mathbf{r}^{\prime}\right) \sigma\left(\mathbf{r}, \mathbf{r}^{\prime}\right) .
$$

Then the Hamiltonian diagonalization leads to the Bogolubov equations

$$
\begin{gathered}
\int\left[\omega\left(\mathbf{r}, \mathbf{r}^{\prime}\right) u_{k}\left(\mathbf{r}^{\prime}\right)+\Delta\left(\mathbf{r}, \mathbf{r}^{\prime}\right) v_{k}\left(\mathbf{r}^{\prime}\right)\right] d \mathbf{r}^{\prime}=\varepsilon_{k} u_{k}(\mathbf{r}), \\
\int\left[\omega^{*}\left(\mathbf{r}, \mathbf{r}^{\prime}\right) v_{k}\left(\mathbf{r}^{\prime}\right)+\Delta^{*}\left(\mathbf{r}, \mathbf{r}^{\prime}\right) u_{k}\left(\mathbf{r}^{\prime}\right)\right] d \mathbf{r}^{\prime}=-\varepsilon_{k} v_{k}(\mathbf{r}) .
\end{gathered}
$$

This is the eigenproblem for the Bogolubov functions $u_{k}$ and $v_{k}$ and the Bogolubov spectrum $\varepsilon_{k}$.

The resulting diagonal Hamiltonian is

$$
H_{B}=E_{B}+\sum_{k} \varepsilon_{k} b_{k}^{\dagger} b_{k},
$$

with the nonoperator term

$$
E_{B}=E_{H F B}-\sum_{k} \varepsilon_{k} \int\left|v_{k}(\mathbf{r})\right|^{2} d \mathbf{r} .
$$

The quasiparticles, described by the operators $b_{k}$, are called bogolons. Their quantum-number distribution is easily calculated, giving

$$
\pi_{k} \equiv\left\langle b_{k}^{\dagger} b_{k}\right\rangle=\left(e^{\beta \varepsilon_{k}}-1\right)^{-1},
$$

which can also be represented as

$$
\pi_{k}=\frac{1}{2}\left[\operatorname{coth}\left(\frac{\varepsilon_{k}}{2 T}\right)-1\right] .
$$

The normal density matrix (88) takes the form

$$
\rho_{1}\left(\mathbf{r}, \mathbf{r}^{\prime}\right)=\sum_{k}\left[\pi_{k} u_{k}(\mathbf{r}) u_{k}^{*}\left(\mathbf{r}^{\prime}\right)+\left(1+\pi_{k}\right) v_{k}^{*}(\mathbf{r}) v_{k}\left(\mathbf{r}^{\prime}\right)\right],
$$

while the anomalous average (89) becomes

$$
\sigma_{1}\left(\mathbf{r}, \mathbf{r}^{\prime}\right)=\sum_{k}\left[\pi_{k} u_{k}(\mathbf{r}) v_{k}^{*}\left(\mathbf{r}^{\prime}\right)+\left(1+\pi_{k}\right) v_{k}^{*}(\mathbf{r}) u_{k}\left(\mathbf{r}^{\prime}\right)\right] .
$$

The density of uncondensed particles (91) is

$$
\rho_{1}(\mathbf{r})=\sum_{k}\left[\pi_{k}\left|u_{k}(\mathbf{r})\right|^{2}+\left(1+\pi_{k}\right)\left|v_{k}(\mathbf{r})\right|^{2}\right]
$$


and the diagonal anomalous average (92) is

$$
\sigma_{1}(\mathbf{r})=\sum_{k}\left(1+2 \pi_{k}\right) u_{k}(\mathbf{r}) v_{k}^{*}(\mathbf{r}) .
$$

The grand thermodynamic potential

$$
\Omega \equiv-T \ln \operatorname{Tr} e^{-\beta H},
$$

under Hamiltonian (231), reads as

$$
\Omega=E_{B}+T \sum_{k} \ln \left(1-e^{-\beta \varepsilon_{k}}\right)
$$

where the first term, defined in Eq. (232), gives

$$
\begin{gathered}
E_{B}=-\frac{1}{2} \int \Phi\left(\mathbf{r}-\mathbf{r}^{\prime}\right)\left[\rho_{0}(\mathbf{r}) \rho_{0}\left(\mathbf{r}^{\prime}\right)+\right. \\
+2 \rho_{0}(\mathbf{r}) \rho_{1}\left(\mathbf{r}^{\prime}\right)+2 \eta^{*}(\mathbf{r}) \eta\left(\mathbf{r}^{\prime}\right) \rho_{1}\left(\mathbf{r}, \mathbf{r}^{\prime}\right)+2 \eta^{*}(\mathbf{r}) \eta^{*}\left(\mathbf{r}^{\prime}\right) \sigma_{1}\left(\mathbf{r}, \mathbf{r}^{\prime}\right)+ \\
\left.+\rho_{1}(\mathbf{r}) \rho_{1}\left(\mathbf{r}^{\prime}\right)+\left|\rho_{1}\left(\mathbf{r}, \mathbf{r}^{\prime}\right)\right|^{2}+\left|\sigma_{1}\left(\mathbf{r}, \mathbf{r}^{\prime}\right)\right|^{2}\right] d \mathbf{r} d \mathbf{r}^{\prime}-\sum_{k} \varepsilon_{k} \int\left|v_{k}(\mathbf{r})\right|^{2} d \mathbf{r}
\end{gathered}
$$

The above equations are valid for any nonuniform matter, with an arbitrary external potential $U(\mathbf{r})$.

\subsection{Uniform Matter}

The previous equations simplify for a uniform case, when there is no external potential. Setting $U=0$, we can use the Fourier transformation (173) and follow the way of Sec. 6 .

Instead of expressions (228) and (229), we now have

$$
\omega_{k} \equiv \frac{k^{2}}{2 m}+\rho \Phi_{0}+\rho_{0} \Phi_{k}+\frac{1}{V} \sum_{p \neq 0} n_{p} \Phi_{k+p}-\mu_{1}
$$

and

$$
\Delta_{k} \equiv \rho_{0} \Phi_{k}+\frac{1}{V} \sum_{p \neq 0} \sigma_{p} \Phi_{k+p}
$$

The HFB Hamiltonian (219) reduces to

$$
H_{H F B}=E_{H F B}+\sum_{k \neq 0} \omega_{k} a_{k}^{\dagger} a_{k}+\frac{1}{2} \sum_{k \neq 0} \Delta_{k}\left(a_{k}^{\dagger} a_{-k}^{\dagger}+a_{-k} a_{k}\right),
$$

with the nonoperator term

$$
E_{H F B}=H^{(0)}-\frac{1}{2} \rho_{1}^{2} \Phi_{0} V-\frac{1}{2 V} \sum_{k p}^{\prime} \Phi_{k+p}\left(n_{k} n_{p}+\sigma_{k} \sigma_{p}\right),
$$

in which $\mathbf{k} \neq 0, \mathbf{p} \neq 0$. 
Instead of the Bogolubov canonical transformations (225), one has

$$
a_{k}=u_{k} b_{k}+v_{-k}^{*} b_{-k}^{\dagger}, \quad b_{k}=u_{k}^{*} a_{k}-v_{k}^{*} a_{-k}^{\dagger} .
$$

And the Bogolubov equations (230) become

$$
\left(\omega_{k}-\varepsilon_{k}\right) u_{k}+\Delta_{k} v_{k}=0, \quad \Delta_{k} u_{k}+\left(\omega_{k}+\varepsilon_{k}\right) v_{k}=0 .
$$

The Bogolubov Hamiltonian (231) has the same form, but with

$$
E_{B}=E_{H F B}+\frac{1}{2} \sum_{k \neq 0}\left(\varepsilon_{k}-\omega_{k}\right)
$$

instead of Eq. (232).

The coefficient functions $u_{k}$ and $v_{k}$ are defined by the Bogolubov equations (247), under conditions

$$
\left|u_{k}\right|^{2}-\left|v_{-k}\right|^{2}=1, \quad u_{k} v_{k}^{*}-v_{-k}^{*} u_{-k}=0,
$$

replacing conditions (226) and (227). These functions, due to the system uniformity and isotropy, are real and symmetric with respect to the momentum inversion $\mathbf{k} \rightarrow-\mathbf{k}$. As a result, one has

$$
\begin{gathered}
u_{k}^{2}-v_{k}^{2}=1, \quad u_{k}^{2}+v_{k}^{2}=\frac{\omega_{k}}{\varepsilon_{k}}, \quad u_{k} v_{k}=-\frac{\Delta_{k}}{2 \varepsilon_{k}}, \\
u_{k}^{2}=\frac{\omega_{k}+\varepsilon_{k}}{2 \varepsilon_{k}}, \quad v_{k}^{2}=\frac{\omega_{k}-\varepsilon_{k}}{2 \varepsilon_{k}} .
\end{gathered}
$$

The Bogolubov spectrum becomes

$$
\varepsilon_{k}=\sqrt{\omega_{k}^{2}-\Delta_{k}^{2}}
$$

As is known from Sec. 6, the spectrum has to be gapless, which gives

$$
\mu_{1}=\rho \Phi_{0}+\frac{1}{V} \sum_{k \neq 0}\left(n_{k}-\sigma_{k}\right) \Phi_{k} .
$$

This is different from the condensate chemical potential (224) that is

$$
\mu_{0}=\rho \Phi_{0}+\frac{1}{V} \sum_{k \neq 0}\left(n_{k}+\sigma_{k}\right) \Phi_{k}
$$

With $\mu_{1}$ from Eq. (252), expression (242) is

$$
\omega_{k}=\frac{k^{2}}{2 m}+\rho_{0} \Phi_{k}+\frac{1}{V} \sum_{p \neq 0}\left(n_{p} \Phi_{k+p}-n_{p} \Phi_{p}+\sigma_{p} \Phi_{p}\right) .
$$

In the long-wave limit, the Bogolubov spectrum (251) is of acoustic form (215), with the sound velocity

$$
c=\sqrt{\frac{\Delta}{m^{*}}}
$$


in which

$$
\Delta \equiv \lim _{k \rightarrow 0} \Delta_{k}=\rho_{0} \Phi_{0}+\frac{1}{V} \sum_{p \neq 0} \sigma_{p} \Phi_{p}
$$

and the effective mass is

$$
m^{*} \equiv \frac{m}{1+\frac{2 m}{V} \sum_{p \neq 0}\left(n_{p}-\sigma_{p}\right) \Phi_{p}^{\prime}}
$$

where

$$
\Phi_{p}^{\prime} \equiv \frac{\partial}{\partial p^{2}} \Phi_{p}
$$

¿From Eqs. (255) and (256), we have

$$
\Delta \equiv m^{*} c^{2}=\rho_{0} \Phi_{0}+\frac{1}{V} \sum_{p \neq 0} \sigma_{p} \Phi_{p}
$$

Hence, expression (254) can be written as

$$
\omega_{k}=m^{*} c^{2}+\frac{k^{2}}{2 m}+\rho_{0}\left(\Phi_{k}-\Phi_{0}\right)+\frac{1}{V} \sum_{p \neq 0} n_{p}\left(\Phi_{k+p}-\Phi_{p}\right) .
$$

Comparing Eqs. (213) and (255) yields

$$
\Sigma_{12}(0,0)=\rho_{0} \Phi_{0}+\frac{1}{V} \sum_{p \neq 0} \sigma_{p} \Phi_{p} .
$$

And from the Hugenholtz-Pines relation (203), with $\mu_{1}$ from Eq. (252), we get

$$
\Sigma_{11}(0,0)=\left(\rho+\rho_{0}\right) \Phi_{0}+\frac{1}{V} \sum_{p \neq 0} n_{p} \Phi_{p} .
$$

Of course, the same Eqs. (260) and (261) can be derived directly from the Green function equations.

The condensate chemical potential (253) can be written as

$$
\mu_{0}=\Sigma_{11}(0,0)+\Sigma_{12}(0,0)-2 \rho_{0} \Phi_{0} .
$$

The difference between Eqs. (252) and (253) takes the form

$$
\mu_{0}-\mu_{1}=2\left[\Sigma_{12}(0,0)-\rho_{0} \Phi_{0}\right],
$$

which again tells us that these chemical potentials are different. They coincide only in the Bogolubov approximation [13,14], when $\Sigma_{12}(0,0)$ equals $\rho_{0} \Phi_{0}$. Then $\mu_{0}$ and $\mu_{1}$ both are also equal to $\rho_{0} \Phi_{0}$ and, hence, to each other.

The momentum distribution (185) is

$$
n_{k}=\frac{\omega_{k}}{2 \varepsilon_{k}} \operatorname{coth}\left(\frac{\varepsilon_{k}}{2 T}\right)-\frac{1}{2}
$$

while the anomalous average (187) reads as

$$
\sigma_{k}=-\frac{\Delta_{k}}{2 \varepsilon_{k}} \operatorname{coth}\left(\frac{\varepsilon_{k}}{2 T}\right)
$$


The grand potential (239) enjoys the same form (240), but with

$$
\begin{gathered}
E_{B}=-\frac{V}{2} \int \Phi(\mathbf{r})\left[\rho^{2}+2 \rho_{0} \rho_{1}(\mathbf{r}, 0)+2 \rho_{0} \sigma_{1}(\mathbf{r}, 0)+\right. \\
\left.+\left|\rho_{1}(\mathbf{r}, 0)\right|^{2}+\left|\sigma_{1}(\mathbf{r}, 0)\right|^{2}\right] d \mathbf{r}+\frac{1}{2} \sum_{k}\left(\varepsilon_{k}-\omega_{k}\right)
\end{gathered}
$$

which can be transformed to

$$
\begin{gathered}
E_{B}=-\frac{N}{2} \rho \Phi_{0}-\rho_{0} \sum_{p}\left(n_{p}+\sigma_{p}\right) \Phi_{p}- \\
-\frac{1}{2 V} \sum_{k p}\left(n_{k} n_{p}+\sigma_{k} \sigma_{p}\right) \Phi_{k+p}+\frac{1}{2} \sum_{k}\left(\varepsilon_{k}-\omega_{k}\right) .
\end{gathered}
$$

\subsection{Local-Density Approximation}

When there exists an external potential $U(\mathbf{r})$ and the system is nonuniform, one can use the equations from Sec. 7.2. It is also possible to resort to the local-density approximation [1$3]$. The local-density, or semi-classical, approximation $[85,86]$ is applicable when the external potential is sufficiently smooth, such that

$$
\left|\frac{l_{0}}{U_{0}} \frac{\partial U(\mathbf{r})}{\partial \mathbf{r}}\right| \ll 1
$$

where $U_{0}$ and $l_{0}$ are the characteristic depth and length of the potential, respectively.

In this approximation, one looks for the solutions of the Bogolubov equations (230), represented as

$$
u_{k}(\mathbf{r})=u(\mathbf{k}, \mathbf{r}) \frac{e^{i \mathbf{k} \cdot \mathbf{r}}}{\sqrt{V}}, \quad v_{k}(\mathbf{r})=v(\mathbf{k}, \mathbf{r}) \frac{e^{i \mathbf{k} \cdot \mathbf{r}}}{\sqrt{V}},
$$

where the functions $u(\mathbf{k}, \mathbf{r})$ and $v(\mathbf{k}, \mathbf{r})$ are assumed to be slowly varying as compared to the exponentials, so that

$$
|\nabla u(\mathbf{k}, \mathbf{r})| \ll k|u(\mathbf{k}, \mathbf{r})|, \quad|\nabla v(\mathbf{k}, \mathbf{r})| \ll k|v(\mathbf{k}, \mathbf{r})|
$$

Then, using the notations

$$
\omega(\mathbf{k}, \mathbf{r}) \equiv \frac{k^{2}}{2 m}+U(\mathbf{r})+2 \Phi_{0} \rho(\mathbf{r})-\mu_{1}(\mathbf{r})
$$

and

$$
\Delta(\mathbf{r}) \equiv\left[\rho_{0}(\mathbf{r})+\sigma_{1}(\mathbf{r})\right] \Phi_{0},
$$

one reduces the Bogolubov equations (230) to the form

$$
\begin{gathered}
{[\omega(\mathbf{k}, \mathbf{r})-\varepsilon(\mathbf{k}, \mathbf{r})] u(\mathbf{k}, \mathbf{r})+\Delta(\mathbf{r}) v(\mathbf{k}, \mathbf{r})=0} \\
\Delta^{*}(\mathbf{r}) u(\mathbf{k}, \mathbf{r})+\left[\omega^{*}(\mathbf{k}, \mathbf{r})+\varepsilon(\mathbf{k}, \mathbf{r})\right] v(\mathbf{k}, \mathbf{r})=0
\end{gathered}
$$

in which

$$
\Phi_{0} \equiv \int \Phi(\mathbf{r}) d \mathbf{r}
$$


The following procedure is analogous to the uniform case. For the coefficient functions, we have

$$
\begin{gathered}
u^{2}(\mathbf{k}, \mathbf{r})-v^{2}(\mathbf{k}, \mathbf{r})=1, \quad u^{2}(\mathbf{k}, \mathbf{r})+v^{2}(\mathbf{k}, \mathbf{r})=\frac{\omega(\mathbf{k}, \mathbf{r})}{\varepsilon(\mathbf{k}, \mathbf{r})}, \\
u(\mathbf{k}, \mathbf{r}) v(\mathbf{k}, \mathbf{r})=-\frac{\Delta(\mathbf{r})}{2 \varepsilon(\mathbf{k}, \mathbf{r})}, \\
u^{2}(\mathbf{k}, \mathbf{r})=\frac{\omega(\mathbf{k}, \mathbf{r})+\varepsilon(\mathbf{k}, \mathbf{r})}{2 \varepsilon(\mathbf{k}, \mathbf{r})}, \quad v^{2}(\mathbf{k}, \mathbf{r})=\frac{\omega(\mathbf{k}, \mathbf{r})-\varepsilon(\mathbf{k}, \mathbf{r})}{2 \varepsilon(\mathbf{k}, \mathbf{r})} .
\end{gathered}
$$

The local Bogolubov spectrum is

$$
\varepsilon(\mathbf{k}, \mathbf{r})=\sqrt{\omega^{2}(\mathbf{k}, \mathbf{r})-\Delta^{2}(\mathbf{r})} .
$$

From the requirement that the spectrum be gapless,

$$
\lim _{k \rightarrow 0} \varepsilon(\mathbf{k}, \mathbf{r})=0,
$$

we find

$$
\mu_{1}(\mathbf{r})=U(\mathbf{r})+\left[\rho_{0}(\mathbf{r})+2 \rho_{1}(\mathbf{r})-\sigma_{1}(\mathbf{r})\right] \Phi_{0} .
$$

Denoting

$$
\Delta(\mathbf{r}) \equiv m c^{2}(\mathbf{r})
$$

from Eq. (272), we get

$$
m c^{2}(\mathbf{r})=\left[\rho_{0}(\mathbf{r})+\sigma_{1}(\mathbf{r})\right] \Phi_{0} .
$$

Then Eq. (271) becomes

$$
\omega(\mathbf{k}, \mathbf{r})=m c^{2}(\mathbf{r})+\frac{k^{2}}{2 m} .
$$

The local Bogolubov spectrum (276) takes the form

$$
\varepsilon(\mathbf{k}, \mathbf{r})=\sqrt{c^{2}(\mathbf{r}) k^{2}+\left(\frac{k^{2}}{2 m}\right)^{2}} .
$$

This shows that $c(\mathbf{r})$ is the local sound velocity.

With spectrum (282), the bogolon momentum distribution (234) reads as

$$
\pi(\mathbf{k}, \mathbf{r})=\frac{1}{2}\left[\operatorname{coth}\left\{\frac{\varepsilon(\mathbf{k}, \mathbf{r})}{2 T}\right\}-1\right] .
$$

In view of the system isotropy, the symmetry properties

$$
\varepsilon(-\mathbf{k}, \mathbf{r})=\varepsilon(\mathbf{k}, \mathbf{r}), \quad \pi(-\mathbf{k}, \mathbf{r})=\pi(\mathbf{k}, \mathbf{r})
$$

are valid.

The single-particle density matrix (235) now transforms into

$$
\rho_{1}\left(\mathbf{r}, \mathbf{r}^{\prime}\right)=\frac{1}{V} \sum_{k} n(\mathbf{k}, \mathbf{r}) e^{i \mathbf{k} \cdot\left(\mathbf{r}-\mathbf{r}^{\prime}\right)}
$$


while the anomalous average (236) becomes

$$
\sigma_{1}\left(\mathbf{r}, \mathbf{r}^{\prime}\right)=\frac{1}{V} \sum_{k} \sigma(\mathbf{k}, \mathbf{r}) e^{i \mathbf{k} \cdot\left(\mathbf{r}-\mathbf{r}^{\prime}\right)}
$$

Here the particle local momentum distribution, replacing Eq. (264), is

$$
n(\mathbf{k}, \mathbf{r})=\frac{\omega(\mathbf{k}, \mathbf{r})}{2 \varepsilon(\mathbf{k}, \mathbf{r})} \operatorname{coth}\left[\frac{\varepsilon(\mathbf{k}, \mathbf{r})}{2 T}\right]-\frac{1}{2}
$$

and, instead of the anomalous average (265), one has

$$
\sigma(\mathbf{k}, \mathbf{r})=-\frac{m c^{2}(\mathbf{r})}{2 \varepsilon(\mathbf{k}, \mathbf{r})} \operatorname{coth}\left[\frac{\varepsilon(\mathbf{k}, \mathbf{r})}{2 T}\right]
$$

The density of uncondensed particles (237) gives

$$
\rho_{1}(\mathbf{r})=\frac{1}{V} \sum_{k} n(\mathbf{k}, \mathbf{r})
$$

and the anomalous average (238) is

$$
\sigma_{1}(\mathbf{r})=\frac{1}{V} \sum_{k} \sigma(\mathbf{k}, \mathbf{r})
$$

The grand potential (239) reads as

$$
\Omega=E_{B}+T \int \ln [1-\exp \{-\beta \varepsilon(\mathbf{k}, \mathbf{r})\}] \frac{d \mathbf{k}}{(2 \pi)^{3}} d \mathbf{r}
$$

Here the first term, after the dimensional regularization of the expression

$$
\int[\varepsilon(\mathbf{k}, \mathbf{r})-\omega(\mathbf{k}, \mathbf{r})] \frac{d \mathbf{k}}{(2 \pi)^{3}}=\frac{16 m^{4}}{15 \pi^{2}} c^{5}(\mathbf{r}),
$$

takes the form

$$
\begin{gathered}
E_{B}=-\frac{\Phi_{0}}{2} \int\left[\rho^{2}(\mathbf{r})+2 \rho_{0}(\mathbf{r}) \rho_{1}(\mathbf{r})+2 \rho_{0}(\mathbf{r}) \sigma_{1}(\mathbf{r})+\rho_{1}^{2}(\mathbf{r})+\sigma_{1}^{2}(\mathbf{r})\right] d \mathbf{r}+ \\
+\frac{8 m^{4}}{15 \pi^{2}} \int c^{5}(\mathbf{r}) d \mathbf{r} .
\end{gathered}
$$

When the system is constrained inside a fixed volume $V$, then the grand potential $\Omega=-P V$ defines the system pressure $P=-\Omega / V$, irrespectively of whether the system is uniform or not. But, when a nonuniform system is confined inside a trapping potential that does not have rigid boundaries constraining the system inside a given volume, then the system pressure cannot be defined as $-\Omega / V$. It is possible, being based on the generalized definition of thermodynamic limit (13), to introduce an effective volume and effective pressure. However, these quantities are different for different potentials and, moreover, they are not uniquely defined even for a given potential, hence, they would have no physical meaning. 
What is well defined for any nonuniform system is the local pressure $p(\mathbf{r})$ that enters the grand potential through the equality

$$
\Omega=-\int p(\mathbf{r}) d \mathbf{r}
$$

For the grand potential (291), the local pressure is

$$
\begin{gathered}
p(\mathbf{r})=-T \int \ln [1-\exp \{-\beta \varepsilon(\mathbf{k}, \mathbf{r})\}] \frac{d \mathbf{k}}{(2 \pi)^{3}}+ \\
+\frac{\Phi_{0}}{2}\left[\rho^{2}(\mathbf{r})+2 \rho_{0}(\mathbf{r}) \rho_{1}(\mathbf{r})+2 \rho_{0}(\mathbf{r}) \sigma_{1}(\mathbf{r})+\rho_{1}^{2}(\mathbf{r})+\sigma_{1}^{2}(\mathbf{r})\right]-\frac{8 m^{4}}{15 \pi^{2}} c^{5}(\mathbf{r}) .
\end{gathered}
$$

Equation (295) can be represented as the sum

$$
p(\mathbf{r})=p_{0}(\mathbf{r})+p_{T}(\mathbf{r})
$$

in which

$$
p_{0}(\mathbf{r})=\left[\rho^{2}(\mathbf{r})-\rho_{0}^{2}(\mathbf{r})\right] \Phi_{0}+\frac{m^{2} c^{4}(\mathbf{r})}{2 \Phi_{0}}-\frac{8 m^{4}}{15 \pi^{2}} c^{5}(\mathbf{r})
$$

and

$$
p_{T}(\mathbf{r})=-T \int \ln [1-\exp \{-\beta \varepsilon(\mathbf{k}, \mathbf{r})\}] \frac{d \mathbf{k}}{(2 \pi)^{3}} .
$$

The latter term, when temperature decreases, tends to zero as

$$
p_{T}(\mathbf{r}) \simeq \frac{T^{4}}{2 \pi^{2} c^{3}(\mathbf{r})} \quad(T \rightarrow 0)
$$

For asymptotically weak interactions, when $\Phi_{0} \rightarrow 0$, Eq. (280), defining the local sound velocity, reduces to

$$
m c^{2}(\mathbf{r}) \simeq \rho_{0}(\mathbf{r}) \Phi_{0}
$$

In that case, the local pressure (295) simplifies to

$$
p(\mathbf{r})=\frac{1}{2} \rho^{2}(\mathbf{r}) \Phi_{0}-T \int \ln [1-\exp \{-\beta \varepsilon(\mathbf{k}, \mathbf{r})\}] \frac{d \mathbf{k}}{(2 \pi)^{3}},
$$

with the local Bogolubov spectrum

$$
\varepsilon(\mathbf{k}, \mathbf{r})=\sqrt{\rho_{0}(\mathbf{r}) \Phi_{0} \frac{k^{2}}{m}+\left(\frac{k^{2}}{2 m}\right)^{2}}
$$

Such local thermodynamic quantities are common for nonuniform systems, both equilibrium [87] and quasiequilibrium $[88,89]$. 


\subsection{Particle Densities}

In the local-density approximation, it is straightforward to find the densities of particles. Thus, the condensate density is

$$
\rho_{0}(\mathbf{r})=|\eta(\mathbf{r})|^{2} .
$$

For an equilibrium system, the condensate function is real. Equation (223) for the condensate function, in the local-density approximation, becomes

$$
\left[-\frac{\nabla^{2}}{2 m}+U(\mathbf{r})\right] \eta(\mathbf{r})+\Phi_{0}\left[\rho_{0}(\mathbf{r})+2 \rho_{1}(\mathbf{r})+\sigma_{1}(\mathbf{r})\right] \eta(\mathbf{r})=\mu_{0} \eta(\mathbf{r}) .
$$

The simplest way of solving this equation is by means of the Thomas-Fermi approximation, when one neglects the spatial derivative, which yields

$$
\rho_{T F}(\mathbf{r})=\frac{\mu_{0}-U(\mathbf{r})}{\Phi_{0}}-2 \rho_{1}(\mathbf{r})-\sigma_{1}(\mathbf{r})
$$

In the case of cylindrical symmetry, one can introduce the Thomas-Fermi volume $V_{T F}=\pi R^{2} L$, with the Thomas-Fermi radius $R$ and the Thomas-Fermi length $L$ defined by the equations

$$
\begin{gathered}
\mu_{0}=U(R, 0)+\Phi_{0}\left[2 \rho_{1}(R, 0)+\sigma_{1}(R, 0)\right] \\
\mu_{0}=U\left(0, \frac{L}{2}\right)+\Phi_{0}\left[2 \rho_{1}\left(0, \frac{L}{2}\right)+\sigma_{1}\left(0, \frac{L}{2}\right)\right] .
\end{gathered}
$$

In the Thomas-Fermi approximation, the condensate density is nonzero only inside the ThomasFermi volume, where

$$
\rho_{0}(\mathbf{r})=\rho_{T F}(\mathbf{r}) \Theta(R-r) \Theta\left(\frac{L}{2}-|z|\right),
$$

with $\Theta(\cdot)$ being the unit step function. Of course, more correctly, the condensate function should be calculated by directly solving Eq. (297).

The density of uncondensed particles (289) can be written as

$$
\rho_{1}(\mathbf{r})=\frac{1}{2} \int\left[\frac{\omega(\mathbf{k}, \mathbf{r})}{\varepsilon(\mathbf{k}, \mathbf{r})}-1\right] \frac{d \mathbf{k}}{(2 \pi)^{3}}+\frac{1}{2} \int \frac{\omega(\mathbf{k}, \mathbf{r})}{\varepsilon(\mathbf{k}, \mathbf{r})}\left\{\operatorname{coth}\left[\frac{\varepsilon(\mathbf{k}, \mathbf{r})}{2 T}\right]-1\right\} \frac{d \mathbf{k}}{(2 \pi)^{3}} .
$$

And the anomalous average (290) is

$$
\sigma_{1}(\mathbf{r})=-\frac{1}{2} \int \frac{m c^{2}(\mathbf{r})}{\varepsilon(\mathbf{k}, \mathbf{r})} \operatorname{coth}\left[\frac{\varepsilon(\mathbf{k}, \mathbf{r})}{2 T}\right] \frac{d \mathbf{k}}{(2 \pi)^{3}} .
$$

At zero temperature, the anomalous average becomes

$$
\sigma_{0}(\mathbf{r})=-\frac{1}{2} \int \frac{m c^{2}(\mathbf{r})}{\varepsilon(\mathbf{k}, \mathbf{r})} \frac{d \mathbf{k}}{(2 \pi)^{3}}
$$

This integral diverges. It can be regularized invoking the dimensional regularization that is well defined for asymptotically weak interactions [4]. Employing the dimensional regularization for finite interactions requires that the limiting condition

$$
\sigma_{0}(\mathbf{r}) \rightarrow 0 \quad\left(\rho_{0} \rightarrow 0\right)
$$


be satisfied $[12,66,67,69-71]$. This condition takes into account that the anomalous averages and Bose condensate always exist together, both being due to the common reason of gauge symmetry breaking. As soon as the condensate density is nonzero, the anomalous average is also nonzero. And, conversely, when the condensate density becomes zero, the anomalous averages have also to disappear.

Another limiting condition is

$$
\sigma_{0}(\mathbf{r}) \rightarrow 0 \quad\left(\Phi_{0} \rightarrow 0\right)
$$

This condition takes into account that the anomalous average nullifies for the ideal Bose gas $[12,66,67,69-71]$.

Under conditions (304) and (305), the dimensional regularization gives

$$
\int \frac{1}{\varepsilon(\mathbf{k}, \mathbf{r})} \frac{d \mathbf{k}}{(2 \pi)^{3}}=-\frac{2 m}{\pi^{2}} \sqrt{m \Phi_{0} \rho_{0}(\mathbf{r})} .
$$

Then Eq. (303) reduces to

$$
\sigma_{0}(\mathbf{r})=\frac{m^{2} c^{2}(\mathbf{r})}{\pi^{2}} \sqrt{m \Phi_{0} \rho_{0}(\mathbf{r})}
$$

Thus, at temperatures outside the critical region, the anomalous average (302) can be represented in the form

$$
\sigma_{1}(\mathbf{r})=\sigma_{0}-\frac{1}{2} \int \frac{m c^{2}(\mathbf{r})}{\varepsilon(\mathbf{k}, \mathbf{r})}\left\{\operatorname{coth}\left[\frac{\varepsilon(\mathbf{k}, \mathbf{r})}{2 T}\right]-1\right\} \frac{d \mathbf{k}}{(2 \pi)^{3}} .
$$

This form can also be used even in the critical region, provided that interactions are weak. Strictly speaking, form (307) is valid when one of the following conditions holds true:

$$
\frac{T}{T_{c}} \ll 1, \quad \frac{\rho \Phi_{0}}{T_{c}} \ll 1,
$$

where $T_{c}$ is the critical temperature.

In the vicinity of the transition point $T_{c}$, where $c(\mathbf{r}) \rightarrow 0$, the anomalous average (302) behaves as

$$
\sigma_{1}(\mathbf{r}) \simeq-\frac{m^{2} T}{2 \pi} c(\mathbf{r}) \quad\left(T \rightarrow T_{c}\right)
$$

This behavior guarantees that the Bose condensation transition is of second order for any interaction strength [12,69-71].

For the convenience of calculations, the density (301) of uncondensed particles can be transformed into

$$
\rho_{1}(\mathbf{r})=\frac{m^{3} c^{3}(\mathbf{r})}{3 \pi^{2}}\left\{1+\frac{3}{2 \sqrt{2}} \int_{0}^{\infty}\left(\sqrt{1+x^{2}}-1\right)^{1 / 2}\left(\operatorname{coth}\left[\frac{m c^{2}(\mathbf{r})}{2 T} x\right]-1\right) d x\right\}
$$

and the anomalous average (307), into

$$
\sigma_{1}(\mathbf{r})=\sigma_{0}(\mathbf{r})-\frac{m^{3} c^{3}(\mathbf{r})}{2 \sqrt{2} \pi^{2}} \int_{0}^{\infty} \frac{\left(\sqrt{1+x^{2}}-1\right)^{1 / 2}}{\sqrt{1+x^{2}}}\left(\operatorname{coth}\left[\frac{m c^{2}(\mathbf{r})}{2 T} x\right]-1\right) d x .
$$

The sound velocity here is defined by Eq. (280). 
The local superfluid density has been introduced in Eq. (155). In the local-density approximation, for an equilibrium system, we have

$$
\rho_{s}(\mathbf{r})=\rho(\mathbf{r})-\frac{2 Q(\mathbf{r})}{3 T}
$$

with the local dissipated heat

$$
Q(\mathbf{r})=\int \frac{k^{2}}{2 m}\left[n(\mathbf{k}, \mathbf{r})+n^{2}(\mathbf{k}, \mathbf{r})-\sigma^{2}(\mathbf{k}, \mathbf{r})\right] \frac{d \mathbf{k}}{(2 \pi)^{3}}
$$

In view of Eqs. (287) and (288), this yields

$$
Q(\mathbf{r})=\frac{1}{(4 \pi)^{2} m} \int_{0}^{\infty} \frac{k^{4} d k}{\sinh ^{2}[\varepsilon(\mathbf{k}, \mathbf{r}) / 2 T]},
$$

which can be transformed into

$$
Q(\mathbf{r})=\frac{m^{4} c^{5}(\mathbf{r})}{(2 \pi)^{2} \sqrt{2}} \int_{0}^{\infty} \frac{\left(\sqrt{1+x^{2}}-1\right)^{3 / 2} x d x}{\sqrt{1+x^{2}} \sinh ^{2}\left[m c^{2}(\mathbf{r}) x / 2 T\right]}
$$

It is necessary to stress the importance of taking account of the anomalous average. If in Eq. (313), one would omit this anomalous average, then the dissipated heat would be infinite, hence

the superfluid density would not exist at all. But, taking the anomalous average into account renders the dissipated heat (314) a well defined finite quantity. The fact that the anomalous average is crucially important for describing superfluidity should be apparent remembering that $\left|\sigma_{1}(\mathbf{r})\right|$ is the density of pair-correlated particles. These pair correlations are, actually, responsible for the existence of superfluidity as such. Therefore, when there are no pair correlations, there is no supefluidity.

Having all particle densities defined makes it possible to study their spatial distributions and to calculate the average condensate, $n_{0}$, and superfluid, $n_{s}$, fractions, as well as the fraction $n_{1}$ of uncondensed particles, given by the equations

$$
\begin{gathered}
n_{0}=\frac{1}{N} \int \rho_{0}(\mathbf{r}) d \mathbf{r}, \quad n_{s}=\frac{1}{N} \int \rho_{s}(\mathbf{r}) d \mathbf{r}, \\
n_{1}=\frac{1}{N} \int \rho_{1}(\mathbf{r}) d \mathbf{r}, \quad n_{0}+n_{1}=1 .
\end{gathered}
$$

\section{Local Interaction Potential}

\subsection{Grand Hamiltonian}

Till now, the consideration, for generality, has been accomplished for any type of the symmetric interaction potential $\Phi(-\mathbf{r})=\Phi(\mathbf{r})$, with the sole restriction that this potential be integrable, such that integral (274), defining $\Phi_{0}$, be finite.

When particles interact with each other through a potential, whose effective interaction radius $r_{0}$ is much shorter than the mean interparticle distance $a$, then this potential can be represented in the local form

$$
\Phi(\mathbf{r})=\Phi_{0} \delta(\mathbf{r}), \quad \Phi_{0} \equiv 4 \pi \frac{a_{s}}{m}
$$


in which the interaction strength $\Phi_{0}$ is expressed through s-wave scattering length $a_{s}$ and mass $m$. For uniform systems, the potential is called stable [90] when $\Phi_{0}$ is positive. For trapped atoms, a finite system can be stable also for negative interactions [1-3,91].

The grand Hamiltonian (67), for the local interaction potential (316), contains the following terms. The zero-order term (68) reads as

$$
H^{(0)}=\int \eta^{*}(\mathbf{r})\left(-\frac{\nabla^{2}}{2 m}+U-\mu_{0}\right) \eta(\mathbf{r}) d \mathbf{r}+\frac{\Phi_{0}}{2} \int|\eta(\mathbf{r})|^{4} d \mathbf{r} .
$$

The first-order term, as always, is zero. The second-order term (70) is

$$
\begin{gathered}
H^{(2)}=\int \psi_{1}^{\dagger}(\mathbf{r})\left(-\frac{\nabla^{2}}{2 m}+U-\mu_{1}\right) \psi_{1}(\mathbf{r}) d \mathbf{r}+ \\
+\Phi_{0} \int\left[2|\eta(\mathbf{r})|^{2} \psi_{1}^{\dagger}(\mathbf{r}) \psi_{1}(\mathbf{r})+\frac{1}{2}\left(\eta^{*}(\mathbf{r})\right)^{2} \psi_{1}(\mathbf{r}) \psi(\mathbf{r})+\frac{1}{2}(\eta(\mathbf{r}))^{2} \psi_{1}^{\dagger}(\mathbf{r}) \psi_{1}^{\dagger}(\mathbf{r})\right] d \mathbf{r} .
\end{gathered}
$$

The third-order term (71) becomes

$$
H^{(3)}=\Phi_{0} \int\left[\eta^{*}(\mathbf{r}) \psi_{1}^{\dagger}(\mathbf{r}) \psi_{1}(\mathbf{r}) \psi_{1}(\mathbf{r})+\psi_{1}^{\dagger}(\mathbf{r}) \psi_{1}^{\dagger}(\mathbf{r}) \psi_{1}(\mathbf{r}) \eta(\mathbf{r})\right] d \mathbf{r}
$$

And the fourth-order term (72) reduces to

$$
H^{(4)}=\frac{\Phi_{0}}{2} \int \psi_{1}^{\dagger}(\mathbf{r}) \psi_{1}^{\dagger}(\mathbf{r}) \psi_{1}(\mathbf{r}) \psi_{1}(\mathbf{r}) d \mathbf{r} .
$$

\subsection{Evolution Equations}

Evolution equations, derived in Sec. 4.5, simplify for the local potential (316). The same notations (90) to (93) can be used. But, instead of (94), we define

$$
\xi(\mathbf{r}) \equiv\left\langle\psi_{1}^{\dagger}(\mathbf{r}) \psi_{1}(\mathbf{r}) \psi_{1}(\mathbf{r})\right\rangle
$$

In addition, we shall employ the notation

$$
\xi_{1}(\mathbf{r}) \equiv\left\langle\psi_{1}(\mathbf{r}) \psi_{1}(\mathbf{r}) \psi_{1}(\mathbf{r})\right\rangle
$$

Equation (95) for the condensate function yields

$$
\begin{gathered}
i \frac{\partial}{\partial t} \eta(\mathbf{r})=\left(-\frac{\nabla^{2}}{2 m}+U-\mu_{0}\right) \eta(\mathbf{r})+ \\
+\Phi_{0}\left[\rho_{0}(\mathbf{r}) \eta(\mathbf{r})+2 \rho_{1}(\mathbf{r}) \eta(\mathbf{r})+\sigma_{1}(\mathbf{r}) \eta^{*}(\mathbf{r})+\xi(\mathbf{r})\right] .
\end{gathered}
$$

The continuity equations (99) to (101) have the same form, but with the source term

$$
\Gamma(\mathbf{r}, t)=i \Phi_{0}\left[\Xi^{*}(\mathbf{r})-\Xi(\mathbf{r})\right]
$$

with the anomalous correlation function

$$
\Xi(\mathbf{r})=\eta^{*}(\mathbf{r})\left[\eta^{*}(\mathbf{r}) \sigma_{1}(\mathbf{r})+\xi(\mathbf{r})\right] .
$$


Equation (85) for the operator of uncondensed particles changes to

$$
i \frac{\partial}{\partial t} \psi_{1}(\mathbf{r}, t)=\left(-\frac{\nabla^{2}}{2 m}+U-\mu_{1}\right) \psi_{1}(\mathbf{r}, t)+\Phi_{0}\left[\hat{X}_{1}(\mathbf{r}, \mathbf{r})+\hat{X}(\mathbf{r}, \mathbf{r})\right]
$$

Equation (103) for the anomalous average becomes

$$
\begin{gathered}
i \frac{\partial}{\partial t} \sigma_{1}(\mathbf{r}, t)=2 K(\mathbf{r})+2\left(U-\mu_{1}\right) \sigma_{1}(\mathbf{r})+ \\
+2 \Phi_{0}\left[\eta^{2}(\mathbf{r}) \rho_{1}(\mathbf{r})+2 \rho_{0}(\mathbf{r}) \sigma_{1}(\mathbf{r})+2 \eta(\mathbf{r}) \xi(\mathbf{r})+\eta^{*}(\mathbf{r}) \xi_{1}(\mathbf{r})+\left\langle\psi_{1}^{\dagger}(\mathbf{r}) \psi_{1}(\mathbf{r}) \psi_{1}(\mathbf{r}) \psi_{1}(\mathbf{r})\right\rangle\right]+ \\
+2\left[\eta^{2}(\mathbf{r})+\sigma_{1}(\mathbf{r})\right] \Phi(0) .
\end{gathered}
$$

The quantity $\Phi(0)$, under the local potential (316), is not defined and requires to be specified by additional constraints.

A straightforward formal way of giving some meaning to this quantity would be by remembering that the delta potential (316) is the limiting form of a potential with a finite interaction range $r_{0}$, such that $r_{0} \ll a$. For instance, potential (316) could be treated as the limiting form of the potential

$$
\Phi(\mathbf{r})=A \exp \left(-\frac{3 r^{2}}{2 r_{0}^{2}}\right)
$$

where $r_{0} \rightarrow 0$, so that the integral

$$
\Phi_{0} \equiv \int \Phi(\mathbf{r}) d \mathbf{r}
$$

is fixed as in Eq. (316). The interaction radius is defined as

$$
r_{0}^{2} \equiv \frac{1}{\Phi_{0}} \int r^{2} \Phi(\mathbf{r}) d \mathbf{r}
$$

These requirements give

$$
A=\left(\frac{3}{2 \pi}\right)^{3 / 2} \frac{\Phi_{0}}{r_{0}^{3}}
$$

Then, for potential (327), the quantity $\Phi(0)$ should be defined as

$$
\Phi(0)=A=3 \sqrt{\frac{6}{\pi}} \frac{a_{s}}{m r_{0}^{3}} .
$$

However, we have to always remember that the local interaction potential (316) is an effective potential modeling particle interactions for the processes occurring at the interparticle distance much larger than the interaction radius. In order to characterize the processes at short distance, one has to use a different effective potential that takes into account particle correlations $[5,81,83]$. The latter, in particular, show that two particles cannot exist at the same spatial point. This is equivalent to saying that $\Phi(0)$ must be set to zero.

The four-operator term can be simplified as

$$
\left\langle\psi_{1}^{\dagger}(\mathbf{r}) \psi_{1}(\mathbf{r}) \psi_{1}(\mathbf{r}) \psi_{1}(\mathbf{r})\right\rangle=3 \rho_{1}(\mathbf{r}) \sigma_{1}(\mathbf{r})
$$

while the three-operator terms are left untouched. 
Then the evolution equation (103) for the anomalous average leads to

$$
\begin{gathered}
i \frac{\partial}{\partial t} \sigma_{1}(\mathbf{r})=2 K(\mathbf{r})+2\left(U-\mu_{1}\right) \sigma_{1}(\mathbf{r})+ \\
+2 \Phi_{0}\left[\eta^{2}(\mathbf{r}) \rho_{1}(\mathbf{r})+2 \rho_{0}(\mathbf{r}) \sigma_{1}(\mathbf{r})+3 \rho_{1}(\mathbf{r}) \sigma_{1}(\mathbf{r})+2 \eta(\mathbf{r}) \xi(\mathbf{r})+\eta^{*}(\mathbf{r}) \xi_{1}(\mathbf{r})\right]
\end{gathered}
$$

with the anomalous kinetic-energy density $K(\mathbf{r})$ given by Eq. (104) and with $\Phi(0)$ set to zero.

The derived evolution equations can be used for studying the initiation of Bose-Einstein condensation and also the decoherence processes in systems with spontaneous symmetry breaking. For finite systems, with $N$ degrees of freedom, coherence can persist [92-95] during the time not longer than that of order $N / T$.

\subsection{Equilibrium Systems}

Considering equilibrium systems, we follow Secs. 6 and 7, substituting in the corresponding equations the local potential (316). The grand Hamiltonian (219), in the HFB approximation, reads as

$$
\begin{gathered}
H_{H F B}=E_{H F B}+\int \psi_{1}^{\dagger}(\mathbf{r})\left(-\frac{\nabla^{2}}{2 m}+U-\mu_{1}\right) \psi_{1}(\mathbf{r}) d \mathbf{r}+ \\
+\Phi_{0} \int\left[2 \rho(\mathbf{r}) \psi_{1}^{\dagger}(\mathbf{r}) \psi_{1}(\mathbf{r})+\frac{1}{2} \sigma(\mathbf{r}) \psi_{1}^{\dagger}(\mathbf{r}) \psi_{1}^{\dagger}(\mathbf{r})+\frac{1}{2} \sigma^{*}(\mathbf{r}) \psi_{1}(\mathbf{r}) \psi_{1}(\mathbf{r})\right] d \mathbf{r}
\end{gathered}
$$

where the nonoperator term is

$$
E_{H F B}=H^{(0)}-\frac{\Phi_{0}}{2} \int\left[2 \rho_{1}^{2}(\mathbf{r})+\sigma_{1}^{2}(\mathbf{r})\right] d \mathbf{r}
$$

and the notation

$$
\rho(\mathbf{r}) \equiv \rho_{0}(\mathbf{r})+\rho_{1}(\mathbf{r}), \quad \sigma(\mathbf{r}) \equiv \eta^{2}(\mathbf{r})+\sigma_{1}(\mathbf{r})
$$

is used. The condensate-function equation (223) becomes

$$
\left(-\frac{\nabla^{2}}{2 m}+U\right) \eta(\mathbf{r})+\Phi_{0}\left[\rho_{0}(\mathbf{r}) \eta(\mathbf{r})+2 \rho_{1}(\mathbf{r}) \eta(\mathbf{r})+\sigma_{1}(\mathbf{r}) \eta^{*}(\mathbf{r})+\xi(\mathbf{r})\right]=\mu_{0} \eta(\mathbf{r}) .
$$

For the condensate chemical potential (224), we have

$$
\begin{gathered}
\mu_{0}=\frac{1}{N_{0}} \int \eta^{*}(\mathbf{r})\left(-\frac{\nabla^{2}}{2 m}+U\right) \eta(\mathbf{r}) d \mathbf{r}+ \\
+\frac{\Phi_{0}}{N_{0}} \int\left[\rho_{0}^{2}(\mathbf{r})+2 \rho_{0}(\mathbf{r}) \rho_{1}(\mathbf{r})+\left(\eta^{*}(\mathbf{r})\right)^{2} \sigma_{1}(\mathbf{r})\right] d \mathbf{r} .
\end{gathered}
$$

Employing the Bogolubov transformations (225) yields the Bogolubov equations

$$
\hat{\omega}(\mathbf{r}) u_{k}(\mathbf{r})+\Delta(\mathbf{r}) v_{k}(\mathbf{r})=\varepsilon_{k} u_{k}(\mathbf{r}), \quad \hat{\omega}(\mathbf{r}) v_{k}(\mathbf{r})+\Delta^{*}(\mathbf{r}) u_{k}(\mathbf{r})=-\varepsilon_{k} v_{k}(\mathbf{r})
$$

replacing Eqs. (230), with the operator

$$
\hat{\omega}(\mathbf{r}) \equiv-\frac{\nabla^{2}}{2 m}+U(\mathbf{r})-\mu_{1}+2 \Phi_{0} \rho(\mathbf{r})
$$


instead of Eq. (228), and with

$$
\Delta(\mathbf{r}) \equiv \Phi_{0}\left[\eta^{2}(\mathbf{r})+\sigma_{1}(\mathbf{r})\right],
$$

instead of Eq. (229). The chemical potential $\mu_{1}$ is defined by the requirement that the spectrum $\varepsilon_{k}$ be gapless.

The grand potential has the same form (240), with

$$
\begin{gathered}
E_{B}=-\frac{\Phi_{0}}{2} \int\left[\rho_{0}^{2}(\mathbf{r})+4 \rho_{0}(\mathbf{r}) \rho_{1}(\mathbf{r})+2 \rho_{1}^{2}(\mathbf{r})+2\left(\eta^{*}(\mathbf{r})\right)^{2} \sigma_{1}(\mathbf{r})+\sigma_{1}^{2}(\mathbf{r})\right] d \mathbf{r}- \\
-\sum_{k} \int \varepsilon_{k}\left|v_{k}(\mathbf{r})\right|^{2} d \mathbf{r},
\end{gathered}
$$

in agreement with Eq. (241).

\subsection{Uniform Systems}

Resorting to the Fourier transformation (173) gives the following terms of the grand Hamiltonian (67). The zero-order term (317) is

$$
H^{(0)}=\left(\frac{1}{2} \rho^{2} \Phi_{0}-\mu_{0} \rho_{0}\right) V .
$$

The first-order term is, as always, zero. The second-order term (318) reads as

$$
H^{(2)}=\sum_{k \neq 0}\left(\frac{k^{2}}{2 m}+2 \rho_{0} \Phi_{0}-\mu_{1}\right) a_{k}^{\dagger} a_{k}+\frac{1}{2} \rho_{0} \Phi_{0} \sum_{k \neq 0}\left(a_{k}^{\dagger} a_{-k}^{\dagger}+a_{-k} a_{k}\right) .
$$

The third-order term (319) yields

$$
H^{(3)}=\sqrt{\frac{\rho_{0}}{V}} \Phi_{0} \sum_{k p}^{\prime}\left(a_{k}^{\dagger} a_{k+p} a_{-p}+a_{-p}^{\dagger} a_{k+p}^{\dagger} a_{k}\right),
$$

where

$$
\mathbf{k} \neq 0, \quad \mathbf{k}+\mathbf{p} \neq 0, \quad \mathbf{p} \neq 0 .
$$

And the fourth-order term (320) becomes

$$
H^{(4)}=\frac{\Phi_{0}}{2 V} \sum_{q} \sum_{k p}^{\prime} a_{k}^{\dagger} a_{p}^{\dagger} a_{p+q} a_{k-q},
$$

in which

$$
\mathbf{k} \neq 0, \quad \mathbf{p} \neq 0, \quad \mathbf{p}+\mathbf{q} \neq 0, \quad \mathbf{k}-\mathbf{q} \neq 0 .
$$

Equation (183) defines the condensate chemical potential

$$
\mu_{0}=\rho \Phi_{0}+\frac{\Phi_{0}}{V} \sum_{k \neq 0}\left(n_{k}+\sigma_{k}+\frac{\xi_{k}}{\sqrt{\rho_{0}}}\right) .
$$


The grand Hamiltonian (332) in the HFB approximation transforms to

$$
H_{H F B}=E_{H F B}+\sum_{k \neq 0} \omega_{k} a_{k}^{\dagger} a_{k}+\frac{\Delta}{2} \sum_{k \neq 0}\left(a_{k}^{\dagger} a_{-k}^{\dagger}+a_{-k} a_{k}\right),
$$

where the nonoperator term (333) is given by the expression

$$
\frac{E_{H F B}}{V}=\frac{\Phi_{0}}{2}\left(\rho_{0}^{2}-2 \rho_{1}^{2}+\sigma_{1}^{2}\right)-\mu_{0} \rho_{0} .
$$

Also, here the notation

$$
\omega_{k}=\frac{k^{2}}{2 m}-\mu_{1}+2 \rho \Phi_{0}
$$

is used, and, instead of Eq. (339), we have

$$
\Delta=\Phi_{0}\left(\rho_{0}+\sigma_{1}\right)
$$

The diagonalization of Hamiltonian (346) is done by the Bogolubov canonical transformations (246), resulting in the Hamiltonian of the Bogolubov form (231).

The condensate chemical potential (253), or (336), reads as

$$
\mu_{0}=\Phi_{0}\left(\rho_{0}+2 \rho_{1}+\sigma_{1}\right) .
$$

And the chemical potential (252) becomes

$$
\mu_{1}=\Phi_{0}\left(\rho_{0}+2 \rho_{1}-\sigma_{1}\right) .
$$

Using the latter in Eq. (348) gives

$$
\omega_{k}=\frac{k^{2}}{2 m}+\Phi_{0}\left(\rho_{0}+\sigma_{1}\right) .
$$

The long-wave spectrum is acoustic,

$$
\varepsilon_{k} \simeq c k \quad(k \rightarrow 0)
$$

with the sound velocity

$$
c \equiv \sqrt{\frac{\Delta}{m}}
$$

which is defined by the equation

$$
m c^{2}=\Phi_{0}\left(\rho_{0}+\sigma_{1}\right) .
$$

Combining Eqs. (352) and (355) yields

$$
\omega_{k}=m c^{2}+\frac{k^{2}}{2 m} .
$$

The solution to the Bogolubov equations (247) results in the Bogolubov spectrum

$$
\varepsilon_{k}=\sqrt{(c k)^{2}+\left(\frac{k^{2}}{2 m}\right)^{2}}
$$


Calculating Eq. (340), we resort to the dimensional regularization giving

$$
\int\left(\varepsilon_{k}-\omega_{k}\right) \frac{d \mathbf{k}}{(2 \pi)^{3}}=\frac{16 m^{4} c^{5}}{15 \pi^{2}}
$$

Then Eq. (340) reduces to

$$
\frac{E_{B}}{V}=-\frac{\Phi_{0}}{2}\left[\rho^{2}+2 \rho_{0}\left(\rho_{1}+\sigma_{1}\right)+\rho_{1}^{2}+\sigma_{1}^{2}\right]+\frac{8 m^{4} c^{5}}{15 \pi^{2}} .
$$

The system pressure can be expressed through the grand potential (240), which gives

$$
p \equiv-\frac{\Omega}{V}=-\frac{E_{B}}{V}-T \int \ln \left(1-e^{-\beta \varepsilon_{k}}\right) \frac{d \mathbf{k}}{(2 \pi)^{3}} .
$$

The integral in Eq. (359) corresponds to thermal pressure. It can be calculated by transforming it to the form

$$
\int \ln \left(1-e^{-\beta \varepsilon_{k}}\right) \frac{d \mathbf{k}}{(2 \pi)^{3}}=\frac{(m c)^{3}}{2 \sqrt{2} \pi^{2}} \int_{0}^{\infty} \ln \left[1-\exp \left(-\frac{m c^{2}}{T} x\right)\right] \frac{\left(\sqrt{1+x^{2}}-1\right)^{1 / 2} x d x}{\sqrt{1+x^{2}}} .
$$

At low temperatures, such that $T \ll m c^{2}$, one can expand the integral as

$$
\int \ln \left(1-e^{-\beta \varepsilon_{k}}\right) \frac{d \mathbf{k}}{(2 \pi)^{3}} \simeq-\frac{(m c)^{3}}{2 \pi^{2}}\left[\left(\frac{T}{m c^{2}}\right)^{3}-\frac{15}{2}\left(\frac{T}{m c^{2}}\right)^{5}\right]
$$

Therefore, the zero-temperature pressure is

$$
p=-\frac{E_{B}}{V} \quad(T=0) .
$$

The internal energy is given by the expression

$$
E \equiv\langle H\rangle+\mu N
$$

in which the average of the grand Hamiltonian is as in Eq. (59) and the system chemical potential is defined in Eq. (60). At zero temperature, the internal energy (361) yields the ground-state energy

$$
E_{0}=E_{B}+\mu N \quad(T=0)
$$

where we take into account that

$$
\langle H\rangle=\left\langle H_{B}\right\rangle=E_{B} \quad(T=0) .
$$

Then the ground-state energy is given by the equation

$$
\frac{E_{0}}{N}=\frac{2 \pi a_{s}}{m \rho}\left(\rho^{2}+\rho_{1}^{2}-2 \rho_{1} \sigma_{1}-\sigma_{1}^{2}\right)+\frac{8 m^{4} c^{5}}{15 \pi^{2} \rho} .
$$

For convenience, let us introduce the dimensionless ground-state energy

$$
e_{0} \equiv \frac{2 m E}{N \rho^{3 / 2}}
$$


and the dimensionless gas parameter

$$
\gamma \equiv \rho^{1 / 3} a_{s} .
$$

The ground-state energy (364) at weak interactions, when $\gamma \ll 1$, allows $[69,70]$ for the expansion

$$
e_{0} \simeq 4 \pi \gamma+\frac{512}{15} \sqrt{\pi} \gamma^{5 / 2}+\frac{512}{9} \gamma^{4} .
$$

The first two terms here reproduce the Lee-Huang-Yang result [96-98]. When particle interactions are strong, so that $\gamma \gg 1$, then [69] one has

$$
e_{0} \simeq 8 \pi \gamma+\frac{6}{5}\left(9 \pi^{4}\right)^{1 / 3}-\frac{3}{4}\left(3 \pi^{5}\right)^{1 / 3} \frac{1}{\gamma}+\frac{1}{64}\left(3 \pi^{8}\right)^{1 / 3} \frac{1}{\gamma^{4}} .
$$

\subsection{Atomic Fractions}

For the local interaction potential (316), it is straightforward to calculate all atomic densities and fractions. The condensate density

$$
\rho_{0}=\rho-\rho_{1}
$$

is expressed through the density of uncondensed particles

$$
\rho_{1}=\int n_{k} \frac{d \mathbf{k}}{(2 \pi)^{3}}=\int\left[\frac{\omega_{k}}{2 \varepsilon_{k}} \operatorname{coth}\left(\frac{\varepsilon_{k}}{2 T}\right)-\frac{1}{2}\right] \frac{d \mathbf{k}}{(2 \pi)^{3}}
$$

that can be rewritten as

$$
\rho_{1}=\frac{1}{2} \int\left(\frac{\omega_{k}}{\varepsilon_{k}}-1\right) \frac{d \mathbf{k}}{(2 \pi)^{3}}+\int \frac{\omega_{k}}{2 \varepsilon_{k}}\left[\operatorname{coth}\left(\frac{\varepsilon_{k}}{2 T}\right)-1\right] \frac{d \mathbf{k}}{(2 \pi)^{3}} .
$$

The anomalous average

$$
\sigma_{1}=\int \sigma_{k} \frac{d \mathbf{k}}{(2 \pi)^{3}}=-\int \frac{m c^{2}}{2 \varepsilon_{k}} \operatorname{coth}\left(\frac{\varepsilon_{k}}{2 T}\right) \frac{d \mathbf{k}}{(2 \pi)^{3}}
$$

can be treated as in Sec. 7, by separating the term

$$
\sigma_{0} \equiv-\int \frac{m c^{2}}{2 \varepsilon_{k}} \frac{d \mathbf{k}}{(2 \pi)^{3}},
$$

which gives

$$
\sigma_{1}=\sigma_{0}-\int \frac{m c^{2}}{2 \varepsilon_{k}}\left[\operatorname{coth}\left(\frac{\varepsilon_{k}}{2 T}\right)-1\right] \frac{d \mathbf{k}}{(2 \pi)^{3}} .
$$

Expression (372) diverges, but can be regularized invoking the dimensional regularization, as in Sec. 7 , resulting in

$$
\int \frac{1}{\varepsilon_{K}} \frac{d \mathbf{k}}{(2 \pi)^{3}}=-\frac{2 m}{\pi^{2}} \sqrt{m \rho_{0} \Phi_{0}} .
$$

Then Eq. (372) becomes

$$
\sigma_{0}=\left(\frac{m c}{\pi}\right)^{2} \sqrt{m \rho_{0} \Phi_{0}}
$$


The anomalous average (373), with the separated term (374), is valid if at least one of conditions (308) is satisfied.

In the vicinity of the condensation temperature $T_{c}$, it is necessary to use the anomalous average in the form

$$
\sigma_{1} \simeq-\frac{m c^{2} T}{2 \pi} \quad\left(T \rightarrow T_{c}\right)
$$

which follows from Eq. (371) and guarantees the second-order phase transition.

The density of uncondensed particles (370) can be represented as

$$
\rho_{1}=\frac{m^{3} c^{3}}{3 \pi^{2}}\left\{1+\frac{3}{2 \sqrt{2}} \int_{0}^{\infty}\left(\sqrt{1+x^{2}}-1\right)^{1 / 2}\left[\operatorname{coth}\left(\frac{m c^{2}}{2 T} x\right)-1\right] d x\right\}
$$

and the anomalous average (373), as

$$
\sigma_{1}=\sigma_{0}-\frac{m^{3} c^{3}}{2 \sqrt{2} \pi^{2}} \int_{0}^{\infty} \frac{\left(\sqrt{1+x^{2}}-1\right)^{1 / 2}}{\sqrt{1+x^{2}}}\left[\operatorname{coth}\left(\frac{m c^{2}}{2 T} x\right)-1\right] d x
$$

The superfluid fraction

$$
n_{s}=1-\frac{2 Q}{3 T}
$$

is expressed through the dissipated heat

$$
Q=\frac{\Delta^{2}(\hat{\mathbf{P}})}{2 m N}=\frac{\left\langle\hat{\mathbf{P}}^{2}\right\rangle}{2 m N} .
$$

In the HFB approximation, we get

$$
Q=\frac{1}{\rho} \int \frac{k^{2}}{2 m}\left(n_{k}+n_{k}^{2}-\sigma_{k}^{2}\right) \frac{d \mathbf{k}}{(2 \pi)^{3}},
$$

which reduces to

$$
Q=\frac{1}{(4 \pi)^{2} m \rho} \int_{0}^{\infty} \frac{k^{4} d \mathbf{k}}{\sinh ^{2}\left(\varepsilon_{k} / 2 T\right)} .
$$

The superfluid fraction (378) leads to the superfluid density

$$
\rho_{s}=\rho-\frac{(m c)^{5}}{6 \sqrt{2} \pi^{2} m T} \int_{0}^{\infty} \frac{\left(\sqrt{1+x^{2}}-1\right)^{3 / 2} x d x}{\sqrt{1+x^{2}} \sinh ^{2}\left(m c^{2} x / 2 T\right)}
$$

The particle densities are related to particle fractions

$$
n_{0} \equiv \frac{\rho_{0}}{\rho}=1-n_{1}, \quad n_{1} \equiv \frac{\rho_{1}}{\rho}, \quad n_{s} \equiv \frac{\rho_{s}}{\rho} .
$$

Also, let us define the dimensionless anomalous average

$$
\sigma \equiv \frac{\sigma_{1}}{\rho}
$$

and the dimensionless sound velocity

$$
s \equiv \frac{m c}{\rho^{1 / 3}} .
$$


At zero temperature, we have

$$
\begin{gathered}
n_{0}=1-\frac{s^{3}}{3 \pi^{2}}, \quad n_{1}=\frac{s^{3}}{3 \pi^{2}}, \quad n_{s}=1, \\
\sigma=\frac{2 s^{2}}{\pi^{3 / 2}} \sqrt{\gamma n_{0}} \quad(T=0) .
\end{gathered}
$$

When interactions are weak, such that $\gamma \ll 1$, then the following expansions are valid for the condensate fraction

$$
n_{0} \simeq 1-\frac{8}{3 \sqrt{\pi}} \gamma^{3 / 2}-\frac{64}{3 \pi} \gamma^{3}-\frac{640}{9 \pi^{3 / 2}} \gamma^{9 / 2},
$$

sound velocity

$$
s \simeq 2 \sqrt{\pi} \gamma^{1 / 2}+\frac{16}{3} \gamma^{2}+\frac{32}{9 \sqrt{\pi}} \gamma^{7 / 2}-\frac{3904}{27 \pi} \gamma^{5},
$$

and the anomalous average

$$
\sigma \simeq \frac{8}{\sqrt{\pi}} \gamma^{3 / 2}+\frac{32}{\pi} \gamma^{3}-\frac{64}{\pi^{3 / 2}} \gamma^{9 / 2} .
$$

As is seen, the anomalous average is three times larger than the normal fraction of uncondensed particles:

$$
\frac{\sigma}{n_{1}} \simeq 3 \quad(\gamma \ll 1)
$$

This emphasizes again that the anomalous average in no way can be neglected for a Bosecondensed system.

For strong interactions, when $\gamma \gg 1$, we find the following expansions for the condensate fraction

$$
n_{0} \simeq \frac{\pi}{64} \frac{1}{\gamma^{3}}-\frac{1}{512}\left(\frac{\pi^{5}}{9}\right)^{1 / 3} \frac{1}{\gamma^{5}},
$$

sound velocity

$$
s \simeq\left(3 \pi^{2}\right)^{1 / 3}-\frac{1}{64}\left(\frac{\pi^{5}}{9}\right)^{1 / 3} \frac{1}{\gamma^{3}}+\frac{1}{1536}\left(\frac{\pi^{7}}{3}\right)^{1 / 3} \frac{1}{\gamma^{5}},
$$

and the anomalous average

$$
\sigma \simeq \frac{(9 \pi)^{1 / 3}}{4} \frac{1}{\gamma}-\frac{\pi}{64} \frac{1}{\gamma^{3}}-\frac{1}{128}\left(\frac{\pi^{4}}{3}\right)^{1 / 3} \frac{1}{\gamma^{4}}+\frac{1}{512}\left(\frac{\pi^{5}}{9}\right)^{1 / 3} \frac{1}{\gamma^{5}} .
$$

Though now the anomalous average is smaller than $n_{1}$, but it is much larger than the condensate fraction:

$$
\frac{\sigma}{n_{0}} \simeq 15.516 \gamma^{2} \quad(\gamma \gg 1)
$$

Thus, the anomalous average is always of crucial importance and can never be neglected.

At low temperatures, such that

$$
\frac{T}{m c^{2}} \ll 1
$$

we find [69-71] the fraction of uncondensed particles

$$
n_{1} \simeq \frac{(m c)^{3}}{3 \pi^{2} \rho}+\frac{(m c)^{3}}{12 \rho}\left(\frac{T}{m c^{2}}\right)^{2},
$$


anomalous average

$$
\sigma \simeq \frac{\sigma_{0}}{\rho}-\frac{(m c)^{3}}{12 \rho}\left(\frac{T}{m c^{2}}\right)^{2},
$$

condensate fraction

$$
n_{0} \simeq 1-\frac{(m c)^{3}}{3 \pi^{2} \rho}-\frac{(m c)^{3}}{12 \rho}\left(\frac{T}{m c^{2}}\right)^{2},
$$

and the superfluid fraction

$$
n_{s} \simeq 1-\frac{2 \pi^{2}(m c)^{3}}{45 \rho}\left(\frac{T}{m c^{2}}\right)^{4} .
$$

Notice that the temperature corrections for $\sigma$ are the same as for $n_{1}$ and $n_{0}$.

Bose-Einstein condensation happens at the temperature

$$
T_{c}=\frac{2 \pi}{m}\left[\frac{\rho}{\zeta(3 / 2)}\right]^{2 / 3} .
$$

In the critical region, where $T \rightarrow T_{c}$, so that

$$
\frac{m c^{2}}{T} \ll 1,
$$

we find [69-71] the expansions

$$
\begin{gathered}
n_{1} \simeq\left(\frac{T}{T_{c}}\right)^{3 / 2}+\frac{(m c)^{3}}{3 \pi^{2} \rho}, \quad \sigma \simeq-\frac{m^{2} c T}{2 \pi \rho}, \quad n_{0} \simeq 1-\left(\frac{T}{T_{c}}\right)^{3 / 2}-\frac{(m c)^{3}}{3 \pi^{2} \rho}, \\
n_{s} \simeq 1-\left(\frac{T}{T_{c}}\right)^{3 / 2}+\frac{\zeta(1 / 2)}{\zeta(3 / 2)}\left(\frac{T}{T_{c}}\right)^{1 / 2} \frac{m c^{2}}{T_{c}} .
\end{gathered}
$$

The superfluid fraction disappears together with the condensate fraction.

Passing to dimensionless quantities, it is convenient to consider the temperature deviation

$$
\tau \equiv 1-\frac{T}{T_{c}} \quad\left(T \leq T_{c}\right)
$$

from the dimensionless transition temperature

$$
t_{c} \equiv \frac{m T_{c}}{\rho^{1 / 3}}=\frac{2 \pi}{[\zeta(3 / 2)]^{2 / 3}}=3.312498 .
$$

Then we obtain

$$
\begin{gathered}
s \simeq \frac{3 \pi}{t_{c}} \tau+\frac{9 \pi}{4 t_{c}}\left(1-\frac{2 \pi}{\gamma t_{c}^{2}}\right) \tau^{2}, \quad n_{1} \simeq 1-\frac{3}{2} \tau+\frac{3}{8} \tau^{2}, \\
\sigma \simeq-\frac{3}{2} \tau+\frac{3}{8}\left(1+\frac{6 \pi}{\gamma t_{c}^{2}}\right) \tau^{2}, \quad n_{0} \simeq \frac{3}{2} \tau-\frac{3}{8} \tau^{2}, \\
n_{s} \simeq \frac{3}{2} \tau-\frac{3}{8}\left(1+\frac{132.411}{t_{c}^{3}}\right) \tau^{2} .
\end{gathered}
$$

As is evident, though the anomalous average tends to zero, as $T \rightarrow T_{c}$, but it is of the same order as the condensate fraction, hence, again the anomalous average cannot be omitted. If it were neglected, the transition would become of first order, which is principally incorrect [67,71]. While accurately taking account of the anomalous average renders the Bose-Einstein condensation the correct second-order transition, as is obvious from expansions (405). 


\section{Disordered Bose Systems}

\subsection{Random Potentials}

The properties of Bose systems can be essentially changed by imposing external spatially random potentials. In this section, the theory is presented for the case when such random potentials are imposed on a uniform system. There is vast literature studying Bose systems inside randomly perturbed periodic lattices (see the review article [12] and recent works [99-101], where further references can be found). But this is a different problem that is not touched in the present section. In several papers (e.g. [102-106]) the influence of weak disorder on a uniform Bose-condensed system has been studied. Strong disorder can be treated by means of numerical Monte Carlo simulations [107]. In the present section, the analytical theory is described, which is valid for arbitrarily strong disorder. The consideration below is based on Refs. [108-110].

The system is described by the grand Hamiltonian

$$
H=\hat{H}-\mu_{0} N_{0}-\mu_{1} \hat{N}_{1}-\hat{\Lambda},
$$

with the energy Hamiltonian

$$
\hat{H}=\int \hat{\psi}^{\dagger}(\mathbf{r})\left[-\frac{\nabla^{2}}{2 m}+U(\mathbf{r})+\xi(\mathbf{r})\right] \hat{\psi}(\mathbf{r}) d \mathbf{r}+\frac{\Phi_{0}}{2} \int \hat{\psi}^{\dagger}(\mathbf{r}) \hat{\psi}^{\dagger}(\mathbf{r}) \hat{\psi}(\mathbf{r}) \hat{\psi}(\mathbf{r}) d \mathbf{r}
$$

containing a random external potential $\xi(\mathbf{r})$. Other notations are the same as in the previous sections.

The random potential, without the loss of generality, can be treated as zero-centered, such that

$$
\langle\langle\xi(\mathbf{r})\rangle\rangle=0 .
$$

The double brackets imply the related stochastic averaging [111]. The random-potential correlations are characterized by the correlation function

$$
\left\langle\left\langle\xi(\mathbf{r}) \xi\left(\mathbf{r}^{\prime}\right)\right\rangle\right\rangle=R\left(\mathbf{r}-\mathbf{r}^{\prime}\right) .
$$

One can use the Fourier transformations

$$
\begin{array}{cc}
\xi(\mathbf{r})=\frac{1}{\sqrt{V}} \sum_{k} \xi_{k} e^{i \mathbf{k} \cdot \mathbf{r}}, & \xi_{k}=\frac{1}{\sqrt{V}} \int \xi(\mathbf{r}) e^{-i \mathbf{k} \cdot \mathbf{r}} d \mathbf{r}, \\
R(\mathbf{r})=\frac{1}{V} \sum_{k} R_{k} e^{i \mathbf{k} \cdot \mathbf{r}}, & R_{k}=\int R(\mathbf{r}) e^{-i \mathbf{k} \cdot \mathbf{r}} d \mathbf{r} .
\end{array}
$$

Then Eq. (409) yields the correlators

$$
\left\langle\left\langle\xi_{k}^{*} \xi_{p}\right\rangle\right\rangle=\delta_{k p} R_{k}, \quad\left\langle\left\langle\xi_{k} \xi_{p}\right\rangle\right\rangle=\delta_{-k p} R_{k} .
$$

The quantum statistical averaging, involving a Hamiltonian $H$, for an operator $\hat{A}$, is denoted as

$$
\langle\hat{A}\rangle_{H} \equiv \frac{\operatorname{Tr} \hat{A} e^{-\beta H}}{\operatorname{Tr} e^{-\beta H}} .
$$

The total averaging, including both the quantum and stochastic averagings, is denoted as

$$
\langle\hat{A}\rangle \equiv\left\langle\left\langle\left(\langle\hat{A}\rangle_{H}\right)\right\rangle\right\rangle .
$$


The grand thermodynamic potential is given by the expression

$$
\Omega \equiv-T\left\langle\left\langle\ln \operatorname{Tr} e^{-\beta H}\right\rangle\right\rangle,
$$

corresponding to the frozen disorder.

In addition to the particle densities, considered in the previous sections, for a random system, it is necessary to introduce one more density. This is the glassy density [108]

$$
\rho_{G} \equiv \frac{1}{V} \int\left\langle\left\langle\left|\left\langle\psi_{1}(\mathbf{r})\right\rangle_{H}\right|^{2}\right\rangle\right\rangle d \mathbf{r} .
$$

With the Fourier transform

$$
\psi_{1}(\mathbf{r})=\frac{1}{\sqrt{V}} \sum_{k \neq 0} a_{k} e^{i \mathbf{k} \cdot \mathbf{r}}
$$

we come to

$$
\rho_{G}=\frac{1}{V} \sum_{k \neq 0}\left\langle\left\langle\left|\alpha_{k}\right|^{2}\right\rangle\right\rangle
$$

where

$$
\alpha_{k} \equiv\left\langle a_{k}\right\rangle_{H}
$$

Because of condition (50), we have

$$
\left\langle\left\langle\alpha_{k}\right\rangle\right\rangle=\left\langle a_{k}\right\rangle=0 .
$$

However, quantity (417) is not zero. The glassy fraction is given by

$$
n_{G} \equiv \frac{\rho_{G}}{\rho}=\frac{1}{N} \int\left\langle\left\langle\left|\left\langle\psi_{1}(\mathbf{r})\right\rangle_{H}\right|^{2}\right\rangle\right\rangle d \mathbf{r},
$$

which can be represented as

$$
n_{G}=\frac{1}{N} \sum_{k \neq 0}\left\langle\left\langle\left|\alpha_{k}\right|^{2}\right\rangle\right\rangle .
$$

To better illustrate the idea of the approach we aim at developing, let us set $U=0$. This will simplify the consideration. Then the grand Hamiltonian writes as

$$
H=\sum_{n=0}^{4} H^{(n)}+H_{\xi}
$$

where the first sum consists of terms (341) to (344), while the last part

$$
H_{\xi}=\rho_{0} \xi_{0} \sqrt{V}+\sqrt{\rho_{0}} \sum_{k \neq 0}\left(a_{k}^{\dagger} \xi_{k}+\xi_{k}^{*} a_{k}\right)+\frac{1}{\sqrt{V}} \sum_{k p(\neq 0)} a_{k}^{\dagger} a_{p} \xi_{k-p}
$$

is due to the presence of the random potential. 


\subsection{Stochastic Decoupling}

The sum in Hamiltonian (421) can be treated in the standard way by resorting to the HFB approximation, as in the previous sections. But the part (422), characterizing the action on particles of the random potential, has to be treated with caution. If one would apply to this part the simple HFB-type approximation

$$
a_{k}^{\dagger} a_{p} \xi_{k-p} \rightarrow\left\langle a_{k}^{\dagger} a_{p}\right\rangle \xi_{k-p}+a_{k}^{\dagger} a_{p}\left\langle\xi_{k-p}\right\rangle-\left\langle a_{k}^{\dagger} a_{p}\right\rangle\left\langle\xi_{k-p}\right\rangle,
$$

then the influence of this part, because of Eq. (408), would reduce to the trivial mean-field form

$$
\frac{1}{N} \sum_{k p(\neq 0)} a_{k}^{\dagger} a_{p} \xi_{k-p} \rightarrow \rho_{1} \xi_{0} \sqrt{V}
$$

containing no nontrivial information on the action of the random potential on particles.

In order not to loose the information on the influence of the random potential, we employ the idea of stochastic decoupling that has been used earlier for taking into account stochastic effects in different systems, such as resonant atoms [112-115] and spin assemblies [116-121].

In the present case, the idea is that the simplification of the third-order expression in the last term of Eq. (422) should include only the quantum statistical averaging, but not the stochastic averaging, thus retaining undisturbed stochastic correlations. This idea can be represented in several equivalent ways. We can write

$$
\left\langle a_{k}^{\dagger} a_{p} \xi_{k-p}\right\rangle=\left\langle\left\langle\alpha_{k}^{*} \alpha_{p} \xi_{k-p}\right\rangle\right\rangle,
$$

which is equivalent to

$$
\left\langle a_{k}^{\dagger} a_{p} \xi_{k-p}\right\rangle_{H}=\alpha_{k}^{*} \alpha_{p} \xi_{k-p} .
$$

In turn, the latter is equivalent to the decoupling

$$
a_{k}^{\dagger} a_{p}=a_{k}^{\dagger} \alpha_{p}+\alpha_{k}^{*} a_{p}-\alpha_{k}^{*} \alpha_{p} .
$$

Then, we introduce [108-110] the nonuniform canonical transformation

$$
a_{k}=u_{k} b_{k}+v_{-k}^{*} b_{-k}^{\dagger}+w_{k} \varphi_{k},
$$

whose coefficient functions are defined so that to diagonalize the grand Hamiltonian (421) in terms of the operators $b_{k}$. The latter are treated as quantum variables with the condition

$$
\left\langle b_{k}\right\rangle_{H}=0 .
$$

The variables $\varphi_{k}$ represent stochastic fields. In view of Eqs. (426) and (427), we have

$$
\alpha_{k} \equiv\left\langle a_{k}\right\rangle_{H}=w_{k} \varphi_{k} .
$$

Diagonalizing Hamiltonian (421) results in the relations

$$
\begin{gathered}
u_{k}^{2}=\frac{\omega_{k}+\varepsilon_{k}}{2 \varepsilon_{k}}, \quad v_{k}^{2}=\frac{\omega_{k}-\varepsilon_{k}}{2 \varepsilon_{k}}, \\
u_{k} v_{k}=-\frac{m c^{2}}{2 \varepsilon_{k}}, \quad w_{k}=-\frac{1}{2 \omega_{k}+m c^{2}},
\end{gathered}
$$


in which

$$
\omega_{k}=\frac{k^{2}}{2 m}+m c^{2}
$$

The Bogolubov spectrum

$$
\varepsilon_{k}=\sqrt{(c k)^{2}+\left(\frac{k^{2}}{2 m}\right)^{2}}
$$

has the standard form, with the sound velocity defined by the equation

$$
m c^{2}=\left(n_{0}+\frac{\sigma_{1}}{\rho}\right) \rho \Phi_{0} .
$$

The stochastic field satisfies the Fredholm equation

$$
\varphi_{k}=\sqrt{\rho_{0}} \xi_{k}-\frac{1}{\sqrt{V}} \sum_{p} \frac{\xi_{k-p} \varphi_{p}}{\omega_{p}+m c^{2}} .
$$

Hamiltonian (421) acquires the diagonal form

$$
H=E_{B}+\sum_{k} \varepsilon_{k} b_{k}^{\dagger} b_{k}+H_{r a n}
$$

where the last term

$$
H_{\text {ran }} \equiv \varphi_{0} \sqrt{N_{0}}
$$

characterizes the explicit influence of the random potential on the system energy.

For the particle momentum distribution (185), we get

$$
n_{k}=\frac{\omega_{k}}{2 \varepsilon_{k}} \operatorname{coth}\left(\frac{\varepsilon_{k}}{2 T}\right)-\frac{1}{2}+\left\langle\left\langle\left|\alpha_{k}\right|^{2}\right\rangle\right\rangle
$$

and for the anomalous average (187),

$$
\sigma_{k}=-\frac{m c^{2}}{2 \varepsilon_{k}} \operatorname{coth}\left(\frac{\varepsilon_{k}}{2 T}\right)+\left\langle\left\langle\left|\alpha_{k}\right|^{2}\right\rangle\right\rangle .
$$

Expressions (436) and (437) possess, as compared with Eqs. (264) and (265), additional terms caused by the random potential. From Eqs. (428) and (429), it follows that

$$
\left\langle\left\langle\left|\alpha_{k}\right|^{2}\right\rangle\right\rangle=\frac{\left\langle\left\langle\left|\varphi_{k}\right|^{2}\right\rangle\right\rangle}{\left(\omega_{k}+m c^{2}\right)^{2}} .
$$

The partial chemical potentials (350) and (351) are of the same form

$$
\mu_{0}=\left(\rho+\rho_{1}+\sigma_{1}\right) \Phi_{0}, \quad \mu_{1}=\left(\rho+\rho_{1}-\sigma_{1}\right) \Phi_{0}
$$

But the quantities

$$
\rho_{1}=\frac{1}{V} \sum_{k \neq 0} n_{k} \quad \sigma_{1}=\frac{1}{V} \sum_{k \neq 0} \sigma_{k}
$$

entering them are now different. The density of uncondensed particles becomes the sum of two terms,

$$
\rho_{1}=\rho_{N}+\rho_{G}
$$


The first term is the normal density

$$
\rho_{N}=\frac{1}{2} \int\left[\frac{\omega_{k}}{\varepsilon_{k}} \operatorname{coth}\left(\frac{\varepsilon_{k}}{2 T}\right)-1\right] \frac{d \mathbf{k}}{(2 \pi)^{3}}
$$

that, as earlier, is due to finite temperature and interactions, while the second term,

$$
\rho_{G}=\int \frac{\left\langle\left\langle\left|\varphi_{k}\right|^{2}\right\rangle\right\rangle}{\left(\omega_{k}+m c^{2}\right)^{2}} \frac{d \mathbf{k}}{(2 \pi)^{3}},
$$

is the glassy density produced by the random potential.

The anomalous average is also the sum of two terms,

$$
\sigma_{1}=\sigma_{N}+\rho_{G}
$$

The first term is

$$
\sigma_{N}=-\frac{1}{2} \int \frac{m c^{2}}{\varepsilon_{k}} \operatorname{coth}\left(\frac{\varepsilon_{k}}{2 T}\right) \frac{d \mathbf{k}}{(2 \pi)^{3}},
$$

while the second term, caused by the presence of the random potential, coincides with the glassy density (442).

The partial chemical potentials (439), with expressions (440) and (443), become

$$
\mu_{0}=\left(\rho+\rho_{N}+\sigma_{N}+2 \rho_{G}\right) \Phi_{0}, \quad \mu_{1}=\left(\rho+\rho_{N}-\sigma_{N}\right) \Phi_{0},
$$

essentially differing from each other.

The superfluid density (378) requires the knowledge of the dissipated heat (379). The latter also reduces to the two-term sum

$$
Q=Q_{N}+Q_{G}
$$

The first term is analogous to Eq. (380) giving

$$
Q_{N}=\frac{1}{8 m \rho^{2}} \int \frac{k^{2}}{\sinh ^{2}\left(\varepsilon_{k} / 2 T\right)} \frac{d \mathbf{k}}{(2 \pi)^{3}} .
$$

And the second term

$$
Q_{G}=\frac{1}{2 m \rho} \int \frac{k^{2}\left\langle\left\langle\left|\varphi_{k}\right|^{2}\right\rangle\right\rangle}{\varepsilon_{k}\left(\omega_{k}+m c^{2}\right)} \operatorname{coth}\left(\frac{\varepsilon_{k}}{2 T}\right) \frac{d \mathbf{k}}{(2 \pi)^{3}}
$$

is the heat dissipated by the glassy fraction. Thus, the superfluid density (378) takes the form

$$
n_{s}=1-\frac{2 Q_{N}}{3 T}-\frac{2 Q_{G}}{3 T}
$$

\subsection{Perturbation-Theory Failure}

Considering the case of weak disorder, it is tempting to resort to perturbation theory with respect to disorder strength. In doing this, one has to keep in mind that such a perturbation theory can fail. Therefore, the results derived by means of perturbation theory may be not reliable. To illustrate this, let us consider the average energy

$$
E_{\text {ran }} \equiv\left\langle H_{\text {ran }}\right\rangle=\left\langle\left\langle\varphi_{0}\right\rangle\right\rangle \sqrt{N_{0}},
$$


related to the random term (435).

Assuming that disorder is weak, one could think that Eq. (433) could be treated perturbatively, by means of the iteration procedure starting with

$$
\varphi_{k}^{(0)}=\sqrt{\rho_{0}} \xi_{k}
$$

The first iteration gives

$$
\varphi_{k}^{(1)}=\sqrt{\rho_{0}} \xi_{k}-\sqrt{\frac{\rho_{0}}{V}} \sum_{p} \frac{\xi_{k-p} \xi_{p}}{\omega_{p}+m c^{2}} .
$$

Using this in Eq. (450) yields

$$
E_{\text {ran }}^{(1)}=-\rho_{0} \sum_{p} \frac{\left\langle\left\langle\left|\xi_{p}\right|^{2}\right\rangle\right\rangle}{\omega_{p}+m c^{2}} .
$$

In view of correlators (411), one gets

$$
E_{\text {ran }}^{(1)}=-\int \frac{N_{0} R_{p}}{\omega_{p}+m c^{2}} \frac{d \mathbf{p}}{(2 \pi)^{3}} .
$$

That is, the direct influence of the random potential would lead to the decrease of the system energy. It is exactly this expression (451) that has been obtained by several authors (see, e.g., [122]) employing perturbation theory.

However, from Eqs. (418) and (428), involving no perturbation theory, it is seen that

$$
\left\langle\left\langle\alpha_{k}\right\rangle\right\rangle=0, \quad\left\langle\left\langle\varphi_{k}\right\rangle\right\rangle=0
$$

Consequently, the random energy (450) is exactly zero:

$$
E_{\text {ran }} \equiv\left\langle H_{\text {ran }}\right\rangle=0
$$

Also, using perturbation theory in calculating sound velocity, some authors (e.g. [122]) find that the speed of sound would increase due to the random potential. Contrary to this, in our theory [108-110], the sound velocity decreases, which looks more natural and is in agreement with other [105] calculations. Really, it looks to be clear that the occurrence of an additional random potential should lead to additional scattering and, hence, to the decrease of sound velocity. Some other contradictions resulting from the use of perturbation theory are illustrated in Refs. $[108,110]$.

Note that all consideration above is valid for any type of disorder characterized by the corresponding correlation function (409).

\subsection{Local Correlations}

To proceed further, let us consider local correlations, described by the delta-correlated disorder, when the correlation function (409) is

$$
R(\mathbf{r})=R_{0} \delta(\mathbf{r})
$$

Then Eq. (411) gives

$$
\left\langle\left\langle\xi_{k}^{*} \xi_{p}\right\rangle\right\rangle=\delta_{k p} R_{0}
$$


The solution to the Fredholm equation (433) can be well approximated [108] by

$$
\varphi_{k}=\frac{\sqrt{\rho_{0}} \xi_{k}}{1+\frac{1}{\sqrt{V}} \sum_{p} \frac{\xi_{p}}{\omega_{p}+m c^{2}}} .
$$

It is convenient to introduce [108] the disorder parameter

$$
\zeta \equiv \frac{a}{l_{l o c}}=\frac{1}{\rho^{1 / 3} l_{l o c}},
$$

being the ratio of the mean interparticle distance versus the localization length

$$
l_{l o c} \equiv \frac{4 \pi}{7 m^{2} R_{0}} .
$$

Employing the self-similar approximation theory [123-132] results in

$$
\left\langle\left\langle\left|\varphi_{k}\right|^{2}\right\rangle\right\rangle=\frac{\rho_{0} R_{0} s^{3 / 7}}{(s-\zeta)^{3 / 7}},
$$

where $s$ is the dimensionless sound velocity (385).

The local disorder, with the delta correlation (453), allows for more straightforward calculations. At the same time, it gives good understanding of the influence of disorder on the system even for the general case of nonlocal disorder. If the random potential $\xi(\mathbf{r})$ is characterized by a finite strength $V_{R}$, with the correlation function (409) having a finite correlation length $l_{R}$, then, to pass to that case, one should make the replacement

$$
R_{0}=V_{R}^{2} l_{R}^{3}
$$

which follows directly from the definition of correlator (409). As a result, the localization length (457) becomes

$$
l_{l o c}=\frac{4 \pi}{7 m^{2} V_{R}^{2} l_{R}^{3}} .
$$

The particle fractions

$$
n_{0} \equiv \frac{\rho_{0}}{\rho}, \quad n_{N} \equiv \frac{\rho_{N}}{\rho}, \quad n_{G} \equiv \frac{\rho_{G}}{\rho}
$$

satisfy the normalization

$$
n_{0}+n_{N}+n_{G}=1 .
$$

Let us define the dimensionless anomalous averages

$$
\sigma \equiv \frac{\sigma_{N}}{\rho}, \quad \frac{\sigma_{1}}{\rho}=\sigma+n_{G} .
$$

Passing to dimensionless quantities, let us use the gas parameter $\gamma$, defined in Eq. (365), dimensionless sound velocity (385), and dimensionless temperature

$$
t \equiv \frac{m T}{\rho^{2 / 3}}
$$


Then, the equation for the sound velocity (432) reads as

$$
s^{2}=4 \pi \gamma\left(1-n_{G}+\sigma\right) .
$$

For the normal fraction of uncondensed particles, we have

$$
n_{N}=\frac{s^{3}}{3 \pi^{2}}\left\{1+\frac{3}{2 \sqrt{2}} \int_{0}^{\infty}\left(\sqrt{1+x^{2}}-1\right)^{1 / 2}\left[\operatorname{coth}\left(\frac{s^{2} x}{2 t}\right)-1\right] d x\right\} .
$$

The glassy fraction is

$$
n_{G}=\frac{\left(1-n_{N}\right) \zeta}{\zeta+7 s^{4 / 7}(s-\zeta)^{3 / 7}} .
$$

And the superfluid fraction becomes

$$
n_{s}=1-\frac{4}{3} n_{G}-\frac{s^{5}}{6 \sqrt{2} \pi^{2} t} \int_{0}^{\infty} \frac{\left(\sqrt{1+x^{2}}-1\right)^{3 / 2} x d x}{\sqrt{1+x^{2}} \sinh ^{2}\left(s^{2} x / 2 t\right)} .
$$

The Bose-Einstein condensation temperature $T_{c}$, in the presence of disorder, decreases linearly with the increasing disorder strength, as compared to the transition temperature $T_{c}^{0}$, given by Eq. (400), for the system without disorder. The relative transition temperature decrease, for $\zeta<1$, follows [108] the law

$$
\delta T_{c} \equiv \frac{T_{c}-T_{c}^{0}}{T_{c}^{0}}=-\frac{2 \zeta}{9 \pi} .
$$

This is in agreement with Monte Carlo simulations [107].

Depending on the relation between the localization length $l_{l o c}$ and the coherence length

$$
l_{c o h} \sim \frac{\int r|\rho(\mathbf{r}, 0)| d \mathbf{r}}{\int|\rho(\mathbf{r}, 0)| d \mathbf{r}}
$$

there can exist three different phases [110].

Superfluid phase exists, when the localization length is larger than the coherence length:

$$
l_{l o c}>l_{c o h} .
$$

In this case, the disorder is yet weak and cannot destroy the system coherence.

Bose glass can occur, when the localization length becomes shorter than the coherence length, but yet larger than the mean interparticle distance:

$$
a<l_{l o c}<l_{c o h} .
$$

Then a kind of granular condensate can exist, being localized in different spatial regions that are separated from each other by the normal nonsuperfluid phase.

Normal glass appears, when the localization length is shorter that the mean interparticle distance:

$$
l_{l o c}<a .
$$

Therefore no coherence between particles can arise, all of them being localized in separate regions of deep random wells. 


\subsection{Bose Glass}

The peculiar phase of the Bose glass is the random mixture of Bose-condensed droplets, localized in different spatial regions that are separated from each other by the normal phase. In the Bose-condensed regions, the gauge symmetry is locally broken, while in the regions of the normal phase, the gauge symmetry is preserved. All these regions are randomly distributed in space and it is even possible that they chaotically change their spatial locations. Also, they are not necessarily compact and may be ramified having fractal geometry [133,134].

Such a randomly mixed system is a particular case of heterophase systems, whose examples are ubiquitous in condensed matter physics. In this respect, it is possible to mention paramagnets with local magnetic ordering revealing spin waves [135-139], many ferroelectrics [140-143] and superconductors [144-149], colossal magnetoresistant materials [150-152], and some other systems reviewed in Refs. [54,153-157].

The typical features of these heterophase materials are: (i) the embryos of one phase inside another are mesoscopic, their characteristic sizes being much larger than the mean interparticle distance but shorter than the system length; (ii) the spatial distribution of the embryos, as well as their shapes are random; (iii) the system, as a whole, is quasiequilibrium, being either stable, or at least metastable, with the lifetime essentially longer than the local equilibration time.

Such materials, with randomly distributed mesoscopic embryos of one phase inside another should be distinguished from systems composed of large stationary domains and from Gibbs mixtures of coexisting macroscopic phases [158]. For the equilibrium macroscopic phases, coexisting with each other, one has to consider the interfacial free energy [159]. The notion of interfacial free energy arises when one considers uniform macroscopic phases, while the mesoscopic heterophase inclusions are nonuniform. For quasiequilibrium mesoscopic embryos, the interfacial regions are not well defined, being often ramified and nonequilibrium. Quasiequilibrium embryos of competing phases are also different from nonequilibrium nuclei arising in kinetic phase transitions $[160,161]$.

A general approach to treating such random heterophase mixtures has been advanced and developed in Refs. [162-171], and summarized in the review articles [54,156,157]. Here, this approach is applied for describing the Bose glass.

Assume that we aim at describing the heterophase mixture of normal uncondensed phase and Bose-condensed phase. The condensed phase exists in the form of mesoscopic embryos surrounded by the normal uncondensed phase. The effective total volume, occupied by each phase is $V_{\nu}$, with the index $\nu=1,2$ enumerating the phases. The geometric weight of each phase is

$$
w_{\nu} \equiv \frac{V_{\nu}}{V} \quad\left(V=V_{1}+V_{2}\right)
$$

where $V$ is the system volume. This weight enjoys the standard probability properties

$$
w_{1}+w_{2}=1, \quad 0 \leq w_{\nu} \leq 1
$$

because of which it can be termed the geometric probability.

The nonuniform mixture of phases, consisting of mesoscopic embryos, requires the use of two types of averages, the statistical averaging over the particle degrees of freedom and the configuration averaging over all admissible random spatial phase configurations. Accomplishing the configuration averaging over phase configurations $[54,156,157]$ results in the appearance of the renormalized Hamiltonian

$$
\widetilde{H}=H_{1} \bigoplus H_{2}
$$


consisting of two terms corresponding to each of the phases. This Hamiltonian is defined on the mixture space

$$
\mathcal{M}=\mathcal{H}_{1} \bigotimes \mathcal{H}_{2}
$$

By its mathematical structure, this space is the tensor product of the weighted Hilbert spaces [54,156,157]. It can be treated as a particular case of a fiber bundle [172].

The effective statistical operator for the random mixture becomes

$$
\hat{\rho}=\hat{\rho}_{1} \bigotimes \hat{\rho}_{2}
$$

with $\hat{\rho}_{\nu}$ being the effective statistical operators for each of the phases.

Thus, after the configuration averaging, we come to the statistical ensemble $\{\hat{\rho}, \mathcal{M}\}$ that is the collection of the partial phase ensembles $\left\{\hat{\rho}_{\nu}, \mathcal{H}_{\nu}\right\}$. The partial ensembles can be called the reduced, or restricted, ensembles, since they are defined on the restricted spaces of microscopic states, typical of the corresponding phase [173-175]. All details are given in the reviews $[54,156,157]$.

The Hamiltonians $H_{\nu}$ are the effective phase Hamiltonians. For the Bose-condensed phase,

$$
H_{1}=\hat{H}_{1}-\mu_{0} N_{0}-\mu_{1} \hat{N}_{1}-\hat{\Lambda}
$$

while for the normal uncondensed phase,

$$
H_{2}=\hat{H}_{2}-\mu_{2} \hat{N}_{2}
$$

where $\hat{N}_{2}$ is the number operator for the normal phase.

The energy Hamiltonian for the Bose-condensed phase reads as

$$
\begin{gathered}
\hat{H}_{1}=w_{1} \int \hat{\psi}^{\dagger}(\mathbf{r})\left[-\frac{\nabla^{2}}{2 m}+U(\mathbf{r})+\xi(\mathbf{r})\right] \hat{\psi}(\mathbf{r}) d \mathbf{r}+ \\
+\frac{w_{1}^{2}}{2} \int \hat{\psi}^{\dagger}(\mathbf{r}) \hat{\psi}^{\dagger}\left(\mathbf{r}^{\prime}\right) \Phi\left(\mathbf{r}-\mathbf{r}^{\prime}\right) \hat{\psi}\left(\mathbf{r}^{\prime}\right) \hat{\psi}(\mathbf{r}) d \mathbf{r} d \mathbf{r}^{\prime}
\end{gathered}
$$

with the Bogolubov-shifted field operator

$$
\hat{\psi}(\mathbf{r})=\eta(\mathbf{r})+\psi_{1}(\mathbf{r}) .
$$

For the normal phase, the energy Hamiltonian is

$$
\begin{gathered}
\hat{H}_{2}=w_{2} \int \psi_{2}^{\dagger}(\mathbf{r})\left[-\frac{\nabla^{2}}{2 m}+U(\mathbf{r})+\xi(\mathbf{r})\right] \psi_{2}(\mathbf{r}) d \mathbf{r}+ \\
+\frac{w_{2}^{2}}{2} \int \psi_{2}^{\dagger}(\mathbf{r}) \psi_{2}^{\dagger}\left(\mathbf{r}^{\prime}\right) \Phi\left(\mathbf{r}-\mathbf{r}^{\prime}\right) \psi_{2}\left(\mathbf{r}^{\prime}\right) \psi_{2}(\mathbf{r}) d \mathbf{r} d \mathbf{r}^{\prime}
\end{gathered}
$$

The interaction potential here is written in the general form. But, in particular, it can take the local form (316).

In the broken-symmetry phase, we have, as earlier, the densities of condensed and uncondensed particles, respectively,

$$
\rho_{0}(\mathbf{r})=|\eta(\mathbf{r})|^{2}, \quad \rho_{1}(\mathbf{r})=\left\langle\psi_{1}^{\dagger}(\mathbf{r}) \psi_{1}(\mathbf{r})\right\rangle .
$$


And in the normal phase, there is only the density of normal particles

$$
\rho_{2}(\mathbf{r})=\left\langle\psi_{2}^{\dagger}(\mathbf{r}) \psi_{2}(\mathbf{r})\right\rangle .
$$

The numbers of particles in the whole heterophase system are written as follows. The number of condensed particles is

$$
N_{0}=w_{1} \int \rho_{0}(\mathbf{r}) d \mathbf{r} .
$$

Here and below, the integration is over the whole system. The number of uncondensed particles is given by the average

$$
N_{1}=\left\langle\hat{N}_{1}\right\rangle=w_{1} \int \rho_{1}(\mathbf{r}) d \mathbf{r}
$$

of the number operator

$$
\hat{N}_{1}=w_{1} \int \psi_{1}^{\dagger}(\mathbf{r}) \psi_{1}(\mathbf{r}) d \mathbf{r} .
$$

The number of normal particles is the average

$$
N_{2}=\left\langle\hat{N}_{2}\right\rangle=w_{2} \int \rho_{2}(\mathbf{r}) d \mathbf{r}
$$

of the number operator

$$
\hat{N}_{2}=w_{2} \int \psi_{2}^{\dagger}(\mathbf{r}) \psi_{2}(\mathbf{r}) d \mathbf{r} .
$$

The total number of particles in the system is the sum

$$
N=N_{0}+N_{1}+N_{2} .
$$

The related fractions of condensed, $n_{0}$, uncondensed, $n_{1}$, and normal, $n_{2}$, particles satisfy the normalization

$$
n_{0}+n_{1}+n_{2}=1 .
$$

The system chemical potential is

$$
\mu=\mu_{0} n_{0}+\mu_{1} n_{1}+\mu_{2} n_{2} .
$$

¿From the condition of equilibrium, it follows [12] that

$$
\mu_{2}=\frac{\mu_{0} n_{0}+\mu_{1} n_{1}}{n_{0}+n_{1}}=\mu .
$$

The grand thermodynamic potential is defined as in the previous sections,

$$
\Omega=-T\left\langle\left\langle\ln \operatorname{Tr} e^{-\beta \widetilde{H}}\right\rangle\right\rangle,
$$

with the double brackets implying the stochastic averaging over the random external potential $\xi(\mathbf{r})$. The geometric weights $w_{\nu}$ are defined to be the minimizers of the grand potential (494), under the normalization condition (475). The latter can be taken into account explicitly by introducing the notation

$$
w_{1} \equiv w, \quad w_{2} \equiv 1-w .
$$


Then the minimization of the grand potential (494) implies

$$
\frac{\partial \Omega}{\partial w}=0, \quad \frac{\partial^{2} \Omega}{\partial w^{2}}>0 .
$$

The first condition gives the equation

$$
\left\langle\left\langle\left(\left\langle\frac{\partial \widetilde{H}}{\partial w}\right\rangle_{\widetilde{H}}\right)\right\rangle\right\rangle=\left\langle\frac{\partial \widetilde{H}}{\partial w}\right\rangle=0
$$

for the weight $w$, while the second, the stability condition

$$
\left(\left\langle\frac{\partial^{2} \widetilde{H}}{\partial w^{2}}\right\rangle\right)>\beta\left\langle\left(\frac{\partial \widetilde{H}}{\partial w}\right)^{2}\right\rangle
$$

Since the right-hand side of inequality (498) is non-negative, the sufficient stability condition is

$$
\left\langle\frac{\partial^{2} \widetilde{H}}{\partial w^{2}}\right\rangle>0
$$

Let us use the notation for the single-particle terms of the condensed phase,

$$
\begin{aligned}
K_{1} \equiv \int & \left\langle\hat{\psi}^{\dagger}(\mathbf{r})\left[-\frac{\nabla^{2}}{2 m}+U(\mathbf{r})+\xi(\mathbf{r})\right] \hat{\psi}(\mathbf{r})\right\rangle d \mathbf{r}- \\
& -\mu_{0} \int \rho_{0}(\mathbf{r}) d \mathbf{r}-\mu_{1} \int \rho_{1}(\mathbf{r}) d \mathbf{r}
\end{aligned}
$$

and the normal phase,

$$
K_{2} \equiv \int\left\langle\psi_{2}^{\dagger}(\mathbf{r})\left[-\frac{\nabla^{2}}{2 m}+U(\mathbf{r})+\xi(\mathbf{r})\right] \psi_{2}(\mathbf{r})\right\rangle d \mathbf{r}-\mu_{2} \int \rho_{2}(\mathbf{r}) d \mathbf{r},
$$

respectively. Similarly, we can define the interaction terms for the condensed phase,

$$
\Phi_{1} \equiv \int\left\langle\hat{\psi}^{\dagger}(\mathbf{r}) \hat{\psi}^{\dagger}\left(\mathbf{r}^{\prime}\right) \Phi\left(\mathbf{r}-\mathbf{r}^{\prime}\right) \hat{\psi}\left(\mathbf{r}^{\prime}\right) \hat{\psi}(\mathbf{r})\right\rangle d \mathbf{r} d \mathbf{r}^{\prime}
$$

and the normal phase,

$$
\Phi_{2} \equiv \int\left\langle\psi_{2}^{\dagger}(\mathbf{r}) \psi_{2}^{\dagger}\left(\mathbf{r}^{\prime}\right) \Phi\left(\mathbf{r}-\mathbf{r}^{\prime}\right) \psi_{2}\left(\mathbf{r}^{\prime}\right) \psi_{2}(\mathbf{r})\right\rangle d \mathbf{r} d \mathbf{r}^{\prime}
$$

In the above expressions (500) and (502), it is assumed that the linear in $\psi_{1}$ terms are omitted, being cancelled by the term $\hat{\Lambda}$ in Hamiltonian (479).

Then Eq. (497) yields the equation for the geometric weight of the condensed phase

$$
w=\frac{\Phi_{2}+K_{2}-K_{1}}{\Phi_{1}+\Phi_{2}}
$$

and the stability condition (499) results in the inequality

$$
\Phi_{1}+\Phi_{2}>0
$$

The latter condition shows that the heterophase mixture can exist only for particles with repulsive interactions. 


\section{Particle Fluctuations and Stability}

\subsection{Stability Conditions}

Fluctuations of observable quantities in statistical systems are characterized by the dispersions of self-adjoint operators corresponding to observables. Let $\hat{A}$ be the operator of an observable quantity given by the statistical average $\langle\hat{A}\rangle$ of this operator. The fluctuations of this observable quantity are quantified by the dispersion

$$
\Delta^{2}(\hat{A}) \equiv\left\langle\hat{A}^{2}\right\rangle-\langle\hat{A}\rangle^{2}
$$

The fluctuations of an observable quantity are called thermodynamically normal when the related dispersion is proportional to $N^{\alpha}$, with $\alpha$ not larger than one. And the fluctuations are termed thermodynamically anomalous if the corresponding dispersion is proportional to $N^{\alpha}$, with $\alpha$ larger than one. In recent literature on Bose systems there has appeared a number of articles claiming the occurrence of thermodynamically anomalous fluctuations of the particle number in Bose systems everywhere below the transition temperature.

In the papers $[63,176-178]$ and reviews $[5,9,12]$, it has been explained that the occurrence of such anomalous fluctuations contradicts the basic principles of statistical physics and that their appearance in some theoretical works is due merely to incorrect calculations. Because of the importance of this problem, it is described below, being based on Refs. [5,9,12,63,176-178].

The ratio of the operator dispersion to its average value quantifies the intensity of the system response to the variation of the considered observable. This response has to be finite in order that the system would be stable with respect to the observable-quantity fluctuations. That is, this ratio has to satisfy the stability condition [12,63,176-178]

$$
0 \leq \frac{\Delta^{2}(\hat{A})}{|\langle\hat{A}\rangle|}<\infty
$$

This condition must hold for all observables and for any statistical system, including thermodynamic limit. For extensive observables, to be considered below, $\langle\hat{A}\rangle \propto N$. The limiting ratio

$$
\chi(\hat{A}) \equiv \lim _{N \rightarrow \infty} \frac{\Delta^{2}(\hat{A})}{N}
$$

has the meaning of the response function related to the variation of the observable represented by the operator $\hat{A}$, and can be called fluctuation susceptibility. Therefore, another form of the stability condition is

$$
0 \leq \chi(\hat{A})<\infty
$$

The number of particles is the observable represented by the number operator $\hat{N}$. Hence, the stability condition with respect to particle fluctuations is

$$
0 \leq \chi(\hat{N})<\infty
$$

¿From the general relations of statistical mechanics and thermodynamics that can be found in almost any course $[49,50,59,60,62,79,81,88,89,90]$, it is easy to show that quantity (508) is really proportional to some physical susceptibility. Being interested in particle fluctuations, one has to consider the dispersion $\Delta^{2}(\hat{N})$. The related physical susceptibility is the isothermal compressibility. This can be defined in any statistical ensemble, as is shown below. 
In the canonical ensemble, where the thermodynamic potential is the free energy $F=$ $F(T, V, N)$, the isothermal compressibility is given by the derivatives

$$
\kappa_{T}=\frac{1}{V}\left(\frac{\partial^{2} F}{\partial V^{2}}\right)_{T N}^{-1}=-\frac{1}{V}\left(\frac{\partial P}{\partial V}\right)_{T N}^{-1} .
$$

In the Gibbs ensemble, with the Gibbs thermodynamic potential $G=G(T, P, N)$, the compressibility is

$$
\kappa_{T}=-\frac{1}{V}\left(\frac{\partial^{2} G}{\partial P^{2}}\right)_{T N}=-\frac{1}{V}\left(\frac{\partial V}{\partial P}\right)_{T N} .
$$

And in the grand canonical ensemble, with the grand thermodynamic potential $\Omega=\Omega(T, V, \mu)$, the compressibility becomes

$$
\kappa_{T}=-\frac{1}{N \rho}\left(\frac{\partial^{2} \Omega}{\partial \mu^{2}}\right)_{T V}=\frac{1}{N \rho}\left(\frac{\partial N}{\partial \mu}\right)_{T V} .
$$

Of course, the value of the compressibility does not depend on the used ensemble, provided that it is correctly defined as a representative ensemble $[54,63,64,71]$. The fact that the compressibility is directly related to particle fluctuations is the most evident in the grand canonical ensemble, where

$$
\kappa_{T}=\frac{\Delta^{2}(\hat{N})}{\rho T N} .
$$

The importance of correctly describing the particle fluctuations is caused by the fact that they define not only the compressibility, but also are connected with several other observable quantities, such as the hydrodynamic sound velocity $s_{T}$,

$$
s_{T}^{2} \equiv \frac{1}{m}\left(\frac{\partial P}{\partial \rho}\right)_{T}=\frac{1}{m \rho \kappa_{T}}=\frac{N T}{m \Delta^{2}(\hat{N})},
$$

and the central structure factor

$$
S(0)=\rho T \kappa_{T}=\frac{T}{m s_{T}^{2}}=\frac{\Delta^{2}(\hat{N})}{N} .
$$

As is seen, the susceptibility $\chi(\hat{N})$ coincides with the structure factor $(516)$.

Is is worth stressing that all expressions (511) to (516) are exact thermodynamic relations that are valid for any stable equilibrium statistical system.

In stable statistical systems, the compressibility, as well as the structure factor, are finite. This is a very well known experimental fact. They can be divergent only at phase transition points, where, as is known, the system is unstable. But everywhere outside of transition points, all these quantities must be finite.

\subsection{Fluctuation Theorem}

The stability condition (509) is necessary in order that the system would be stable with respect to the fluctuations of the observable quantity represented by the operator $\hat{A}$. But what can be said with regard to an observable represented by a composite operator

$$
\hat{A}=\sum_{i} \hat{A}_{i},
$$


given by a sum of several self-adjoint operators? How the fluctuations for the total sum of $\hat{A}$ are connected with partial fluctuations for $\hat{A}_{i}$ ? To formulate this question more precisely, let us give some definitions.

\section{Definition: Thermodynamically normal fluctuations}

Fluctuations of an observable quantity, represented by a self-adjoint operator $\hat{A}$, are called thermodynamically normal if and only if the stability condition (509) holds for this operator. Then the related susceptibility (508) is also called thermodynamically normal.

\section{Definition: Thermodynamically anomalous fluctuations}

Fluctuations of an observable quantity, represented by a self-adjoint operator $\hat{A}$, are called thermodynamically anomalous if and only if the stability condition (509) does not hold for this operator. Then the related susceptibility (508) is also termed thermodynamically anomalous.

The question of interest is how the total fluctuation susceptibility $\chi(\hat{A})$ is connected with the partial fluctuation susceptibilities $\chi\left(\hat{A}_{i}\right)$ ? Or, in physical terminology, can it happen that the total susceptibility be finite, while some of the partial susceptibilities be infinite? The answer to this question is given by the following theorem on fluctuations of composite observables.

Fluctuation Theorem.(Yukalov [63,177])

Let the observable quantity be represented by a composite operator (517) that is a sum of self-adjoint operators. Then the dispersion of this operator is

$$
\Delta^{2}\left(\sum_{i} \hat{A}_{i}\right)=\sum_{i} \Delta^{2}\left(\hat{A}_{i}\right)+\sum_{i \neq j} \lambda_{i j} \sqrt{\Delta^{2}\left(\hat{A}_{i}\right) \Delta^{2}\left(\hat{A}_{j}\right)},
$$

where $\left|\lambda_{i j}\right|<1$, hence the total fluctuation susceptibility reads as

$$
\chi\left(\sum_{i} \hat{A}_{i}\right)=\sum_{i} \chi\left(\hat{A}_{i}\right)+\sum_{i \neq j} \lambda_{i j} \sqrt{\chi\left(\hat{A}_{i}\right) \chi\left(\hat{A}_{j}\right)} .
$$

From here it follows that the total fluctuation susceptibility is normal if and only if all partial fluctuation susceptibilities are normal. And the total fluctuation susceptibility is anomalous if and only if at least one of the partial fluctuation susceptibilities is anomalous.

\subsection{Ideal-Gas Instability}

For illustrative purpose, one often considers the ideal Bose gas. The grand Hamiltonian for noninteracting particles is a particular case of Hamiltonian (58), where the energy Hamiltonian is

$$
\hat{H}=\int \hat{\psi}^{\dagger}(\mathbf{r})\left(-\frac{\nabla^{2}}{2 m}+U\right) \hat{\psi}(\mathbf{r}) d \mathbf{r} .
$$

Substituting here the Bogolubov shift (48) yields the grand Hamiltonian

$$
H=\int \eta^{*}(\mathbf{r})\left(-\frac{\nabla^{2}}{2 m}+U-\mu_{0}\right) \eta(\mathbf{r}) d \mathbf{r}+\int \psi_{1}^{\dagger}(\mathbf{r})\left(-\frac{\nabla^{2}}{2 m}+U-\mu_{1}\right) \psi_{1}(\mathbf{r}) d \mathbf{r}
$$

The equations of motion become

$$
i \frac{\partial}{\partial t} \eta(\mathbf{r}, t)=\left(-\frac{\nabla^{2}}{2 m}+U-\mu_{0}\right) \eta(\mathbf{r}, t)
$$




$$
i \frac{\partial}{\partial t} \psi_{1}(\mathbf{r}, t)=\left(-\frac{\nabla^{2}}{2 m}+U-\mu_{1}\right) \psi_{1}(\mathbf{r}, t) .
$$

Let us pass to the uniform gas, when there is no external potential, $U=0$. Then, in equilibrium, the condensate function is constant, $\eta(\mathbf{r}, t)=\eta=$ const. The equation for the condensate function gives $\mu_{0}=0$.

In the momentum representation, Hamiltonian (521), with $U=0$, reduces to

$$
H=\sum_{k \neq 0}\left(\frac{k^{2}}{2 m}-\mu_{1}\right) a_{k}^{\dagger} a_{k} .
$$

The condition of the condensate existence (15), as well as the Hugenholtz-Pines relation (203), result in $\mu_{1}=0$ for temperatures below the condensation temperature

$$
T_{c}=\frac{2 \pi}{m}\left[\frac{\rho}{\zeta(d / 2)}\right]^{d / 2},
$$

written here for a $d$-dimensional space. Expression (524) shows that positive $T_{c}$ does not exist for $d=1$, since $\zeta(1 / 2)=-1.460$ and that $T_{c}=0$ for $d=2$, since $\zeta(1)=\infty$. Positive $T_{c}$ exists only for $d>2$.

For the number operator $\hat{N}=N_{0}+\hat{N}_{1}$, taking into account that $\operatorname{cov}\left(N_{0}, \hat{N}_{1}\right)=0$ and $\Delta^{2}\left(N_{0}\right)=0$, one finds

$$
\Delta^{2}(\hat{N})=\Delta^{2}\left(\hat{N}_{1}\right)
$$

Let us emphasize that the condensate fraction does not fluctuate at all and that the total fluctuations are caused solely by the uncondensed particles. The number operator of the latter is

$$
\hat{N}_{1} \equiv \int \psi_{1}^{\dagger}(\mathbf{r}) \psi_{1}(\mathbf{r}) d \mathbf{r}=\sum_{k \neq 0} a_{k}^{\dagger} a_{k} .
$$

Invoking the commutation relations and the Wick theorem, one has

$$
\begin{gathered}
\left\langle a_{k}^{\dagger} a_{k} a_{p}^{\dagger} a_{p}\right\rangle=\left\langle a_{k}^{\dagger} a_{p}^{\dagger} a_{k} a_{p}\right\rangle+\delta_{k p} n_{k}, \\
\left\langle a_{k}^{\dagger} a_{p}^{\dagger} a_{k} a_{p}\right\rangle=n_{k} n_{p}+\delta_{k p} n_{k}^{2},
\end{gathered}
$$

where the momentum distribution is

$$
n_{k} \equiv\left\langle a_{k}^{\dagger} a_{k}\right\rangle=\left[\exp \left(\frac{\beta k^{2}}{2 m}\right)-1\right]^{-1} .
$$

This leads to

$$
\left\langle\hat{N}_{1}^{2}\right\rangle=N_{1}^{2}+\sum_{k \neq 0} n_{k}\left(1+n_{k}\right) .
$$

Therefore particle fluctuations are characterized by the dispersion

$$
\Delta^{2}(\hat{N})=\Delta^{2}\left(\hat{N}_{1}\right)=\sum_{k \neq 0} n_{k}\left(1+n_{k}\right) .
$$

Remark. In some works, the authors forget that Bose-Einstein condensation necessarily requires broken gauge symmetry. Forgetting this, one extends the sum in Eq. (526) to $k=0$. 
Then, separating the term with $k=0$, one gets the condensate fluctuations described by the dispersion $\Delta^{2}\left(N_{0}\right)$ proportional to $N_{0}^{2}$. One blames the grand canonical ensemble to be guilty for this unreasonable result, naming this "grand canonical catastrophe". However, as is clear, there is no any catastrophe here and not the grand ensemble is guilty, but the authors doing incorrect calculations. One should not forget that, if the gauge symmetry is not broken, then $N_{0} \equiv 0$.

Summing the right-hand side of Eq. (527) yields

$$
\Delta^{2}\left(\hat{N}_{1}\right)=\left(\frac{m T}{\pi}\right)^{2} V^{4 / 3}
$$

This gives the fluctuation susceptibility

$$
\chi(\hat{N})=\chi\left(\hat{N}_{1}\right)=\lim _{N \rightarrow \infty}\left(\frac{m a^{2} T}{\pi}\right)^{2} N^{1 / 3}=\infty .
$$

Consequently, the stability condition (510) does not hold. This means that the ideal uniform Bose-condensed gas is not stable. It is a pathological object that cannot exist in reality.

\subsection{Trapped Atoms}

But maybe the ideal Bose-condensed gas could be stabilized by confining it inside a trap formed by a trapping external potential? A general expression for such a trapping potential is given by the power-law form

$$
U(\mathbf{r})=\sum_{\alpha=1}^{d} \frac{\omega_{\alpha}}{2}\left|\frac{r_{\alpha}}{l_{\alpha}}\right|^{n_{\alpha}}
$$

which is written here in the $d$-dimensional space. The trapping frequency $\omega_{\alpha}$ and the trapping length $l_{\alpha}$ are connected by the relations

$$
\omega_{\alpha}=\frac{1}{m l_{\alpha}^{2}}, \quad l_{\alpha}=\frac{1}{\sqrt{m \omega_{\alpha}}} .
$$

It is also useful to introduce the effective frequency and effective length by the geometric averages

$$
\omega_{0} \equiv\left(\prod_{\alpha=1}^{d} \omega_{\alpha}\right)^{1 / d}=\frac{1}{m l_{0}^{2}}, \quad l_{0} \equiv\left(\prod_{\alpha=1}^{d} l_{\alpha}\right)^{1 / d}=\frac{1}{\sqrt{m \omega_{0}}} .
$$

Let us define the confining dimension [51]

$$
s \equiv \frac{d}{2}+\sum_{\alpha=1}^{d} \frac{1}{n_{\alpha}} .
$$

Passing from the trapping potential to the uniform case implies the limits

$$
n_{\alpha} \rightarrow \infty, \quad l_{0} \rightarrow \frac{L}{2}, \quad \prod_{\alpha=1}^{d} 2 l_{\alpha} \rightarrow L^{d}
$$

where $L$ is the linear size of the system volume $V=L^{d}$. As a result, $s \rightarrow d / 2$, that is, $s$ becomes semi-dimension. 
Bose-Einstein condensation of the ideal Bose gas in the trapping potential (530) can be described employing the generalized quasi-classical approximation [51]. The condensation temperature reads as

$$
T_{c}=\left[\frac{b N}{g_{s}(1)}\right]^{1 / s}
$$

where we use the notation

$$
b \equiv \frac{\pi^{d / 2}}{2^{s}} \prod_{\alpha=1}^{d} \frac{\omega_{\alpha}^{1 / 2+1 / n_{\alpha}}}{\Gamma\left(1+1 / n_{\alpha}\right)}
$$

and introduce the generalized Bose function

$$
g_{s}(z) \equiv \frac{1}{\Gamma(s)} \int_{u_{0}}^{\infty} \frac{z u^{s-1}}{e^{u}-z} d u,
$$

in which the integration is limited from below by the value

$$
u_{0} \equiv \frac{\omega_{0}}{2 T} .
$$

Recall that in the standard Bose function, the integration starts from zero.

The value $g_{s}(1)$ of the generalized function (535) is finite for all $s$ on the complex plane, since $\Gamma(s) \neq 0$, so that $1 / \Gamma(s)$ is an entire function. But $\Gamma(s)$ can be negative for $s<0$, e.g., it is negative in the interval $-1<s<0$. Therefore, $g_{s}(1)$ is positive and finite for all $s>0$. Contrary to this, the standard Bose function would diverge for $s \leq 1$, and there would be no finite condensation temperatures for these $s$. While, in the case of the generalized function (535), finite condensation temperatures formally exist for any positive $s$. Below $T_{c}$, and for $s>0$, the condensate fraction is

$$
n_{0}=1-\left(\frac{T}{T_{c}}\right)^{s} \quad\left(T \leq T_{c}\right) .
$$

The most often studied trapping potential is the harmonic potential, for which $n_{\alpha}=2$ and $s=d$. Then the condensation temperatures are

$$
\begin{gathered}
T_{c}=\frac{N \omega_{0}}{\ln (2 N)} \quad(d=1), \\
T_{c}=\omega_{0}\left[\frac{N}{\zeta(d)}\right]^{1 / d} \quad(d \geq 2) .
\end{gathered}
$$

The condensation temperature (534) is finite for any finite $N$. But it is necessary to check whether it is finite in thermodynamic limit, when $N \rightarrow \infty$. For confined systems, the effective thermodynamic limit is defined [51] in Eq. (13). As an extensive observable, we can take the internal energy that, in the present case, below $T_{c}$, is

$$
E_{N}=\frac{s}{b} g_{1+s}(1) T^{1+s}
$$

Then, the thermodynamic limit (13) reads as

$$
N \rightarrow \infty, \quad E_{N} \rightarrow \infty, \quad \frac{E_{N}}{N} \rightarrow \text { const }
$$


The value $g_{1+s}$ is finite for $N \rightarrow \infty$ at all $s>0$. Hence, Eq. (540) can be rewritten as the limit

$$
N \rightarrow \infty, \quad b \rightarrow 0, \quad b N \rightarrow \text { const } .
$$

For the equipower traps, for which $n_{\alpha}=n$, the effective thermodynamic limit (541) takes the form

$$
N \rightarrow \infty, \quad \omega_{0} \rightarrow 0, \quad N \omega_{0}^{s} \rightarrow \text { const } .
$$

Considering the thermodynamic limit for the condensation temperature (534), we have to take into account that the generalized function (535) yields

$$
\begin{gathered}
g_{s}(1) \cong \frac{1}{(1-s) \Gamma(s)}\left(\frac{2 T}{\omega_{0}}\right)^{1-s} \quad(0<s<1), \\
g_{s}(1) \cong \ln \left(\frac{2 T}{\omega_{0}}\right) \quad(s=1) .
\end{gathered}
$$

Consequently, for the condensation temperature (534), as $N \rightarrow \infty$, we find

$$
\begin{array}{cc}
T_{c} \propto \frac{1}{N^{(1-s) / s}} \rightarrow 0 & (0<s<1), \\
T_{c} \propto \frac{1}{\ln N} \rightarrow 0 & (s=1) \\
T_{c} \rightarrow \text { const } & (s>1) .
\end{array}
$$

Therefore, finite condensation temperatures exist only for $s>1$. This implies that for harmonic traps, for which $s=d$, the finite condensation temperature occurs only for $d \geq 2$. Bose-Einstein condensation cannot happen in one-dimensional harmonic traps at finite temperature.

But this is not yet the whole story. As we know from Sec. 10.3, a finite condensation temperature can formally occur, however, the condensed system in reality is unstable, thus, cannot exist. To check the stability, it is necessary to consider the system susceptibilities. Specific heat for the Bose-condensed trapped gas is finite at all temperatures, displaying a jump at the transition point [51]. We need to consider the isothermic compressibility (514) that shows the system response with respect to particle fluctuations. The dispersion for the number operators behaves as

$$
\Delta^{2}\left(N_{0}\right)=0, \quad \Delta^{2}(\hat{N})=\Delta^{2}\left(\hat{N}_{1}\right) .
$$

It is convenient to introduce the finite- $N$ susceptibility

$$
\chi_{N} \equiv \frac{\Delta^{2}(\hat{N})}{N},
$$

whose limit

$$
\lim _{N \rightarrow \infty} \chi_{N}=\chi(\hat{N})
$$

yields the susceptibility defined in Eq. (508). Below $T_{c}$, we obtain

$$
\chi_{N}=\frac{g_{s-1}(1)}{g_{s}(1)}\left(\frac{T}{T_{c}}\right)^{s} .
$$


Susceptibility (547) is negative for $s<1$ and does not satisfy the stability condition (510). For the values of $s \geq 1$, we have

$$
\begin{gathered}
\chi_{N}=\frac{2}{N}\left(\frac{T}{T_{c}}\right)^{2} \quad(s=1), \\
\chi_{N}=\frac{1}{(2-s) \zeta(s) \Gamma(s-1)}\left(\frac{2 T}{\omega_{0}}\right)^{2-s}\left(\frac{T}{T_{c}}\right)^{2}, \quad(1<s<2), \\
\chi_{N}=\frac{1}{\zeta(2)}\left(\frac{T}{T_{c}}\right)^{2} \ln \left(\frac{2 T}{\omega_{0}}\right) \quad(s=2), \\
\chi_{N}=\frac{\zeta(s-1)}{\zeta(s)}\left(\frac{T}{T_{c}}\right)^{s} \quad(s>2) .
\end{gathered}
$$

For asymptotically large $N$, we get

$$
\begin{array}{cl}
\chi_{N} \propto N & (s=1), \\
\chi_{N} \propto N^{(2-s) / s} & (1<s<2), \\
\chi_{N} \propto \ln N & (s=2), \\
\chi_{N} \propto \text { const } & (s>2) .
\end{array}
$$

This shows that the trapped Bose gas is stable only for $s>2$, when the stability condition (510) is satisfied, that is, when

$$
s \equiv \frac{d}{2}+\sum_{\alpha=1}^{d} \frac{1}{n_{\alpha}}>2 .
$$

In particular, for harmonic traps, for which $s=d$ and $b=\omega^{d}$, one finds

$$
\begin{gathered}
\chi_{N}=\frac{2}{N}\left(\frac{T}{\omega_{0}}\right)^{2} \quad(d=1) . \\
\chi_{N}=\frac{1}{N}\left(\frac{T}{\omega_{0}}\right)^{2} \ln \left(\frac{2 T}{\omega_{0}}\right) \quad(d=2) . \\
\chi_{N}=\frac{\pi^{2}}{6 \zeta(3)}\left(\frac{T}{T_{c}}\right)^{3} \quad(d=3) .
\end{gathered}
$$

For large $N$, this gives

$$
\begin{array}{cc}
\chi_{N} \propto N & (d=1) . \\
\chi_{N} \propto \ln N & (d=2) . \\
\chi_{N} \propto \text { const } & (d=3) .
\end{array}
$$

Thus, the Bose-condensed gas in a harmonic trap is stable only in the three-dimensional space, $d=3$.

The above analysis demonstrates that confining the ideal Bose gas in a trap may stabilize it, which, however, depends on the confining dimension $s$, defined in Eq. (533). The occurrence of a formal expression for the critical temperature $T_{c}$ is not yet sufficient for claiming the possibility of Bose-Einstein condensation in a trapped gas, but it is also necessary to check the system stability. For example, in the case of the power-law trapping potentials, the condensation temperature formally exists for $s>1$. But the trapped condensed gas can be stable only for $s>2$. Oneand two-dimensional harmonic traps are not able to stabilize the condensate. Only the threedimensional harmonic trap is able to host the ideal Bose-condensed gas. 


\subsection{Interacting Systems}

Ideal gases are, actually, rather artificial objects, since there always exist particle interactions, though, maybe, weak. Now we pass to studying the stability of interacting systems.

To a great surprise, there have been published many papers, in which the authors claim that interacting Bose-condensed systems, both uniform as well as trapped, exhibit thermodynamically anomalous particle fluctuations of the same kind as the ideal Bose gas, with the number-operator dispersion (528). By the Bogolubov theorem, one always has $\Delta^{2}\left(\hat{N}_{0}\right)=0$, hence, $\Delta^{2}(\hat{N})=$ $\Delta^{2}\left(\hat{N}_{1}\right)$. Then the thermodynamically anomalous dispersion $\Delta^{2}(\hat{N}) \propto N^{4 / 3}$ would lead to $\chi_{N} \propto$ $N^{1 / 3}$ and to the divergence of $\chi(\hat{N}) \rightarrow \infty$. In that case, the stability condition (510) is not satisfied, and the behavior of all physical quantities would be rather wild. Then the isothermal compressibility (514) would diverge, the sound velocity (515) would be zero, and the structure factor (516) would be infinite. That is, the system would be absolutely unstable.

Moreover, this would mean that any system with spontaneously broken gauge symmetry would not exist. Clearly, such a strange conclusion would contradict all known experiments observing Bose-Einstein condensed trapped gases. Superfluid helium is also the system with broken gauge symmetry, hence, it also would not be able to exist, which is evidently absurd.

In Refs. [5,9,12,63,176-178], it has been explained that the occurrence, in some works, of thermodynamically anomalous fluctuations is caused by incorrect calculations. One calculates the dispersion $\Delta^{2}\left(\hat{N}_{1}\right)$ invoking the Bogolubov approximation that is a second-order approximation with respect to the operators of uncondensed particles. But the expression $\hat{N}_{1}^{2}$ is of fourth order with respect to these operators. Calculating the fourth-order terms in the second-order approximation, strictly speaking, is not self-consistent and can lead to unreasonable results, such as the occurrence of thermodynamically anomalous particle fluctuations.

The correct calculation of the dispersion $\Delta^{2}(\hat{N})$ and, hence, of susceptibility (546), can be done as follows. From the definition of the particle dispersion $\Delta^{2}(\hat{N})$, one has the exact expression

$$
\chi_{N}=1+\frac{1}{N} \int \rho(\mathbf{r}) \rho\left(\mathbf{r}^{\prime}\right)\left[g\left(\mathbf{r}, \mathbf{r}^{\prime}\right)-1\right] d \mathbf{r} d \mathbf{r}^{\prime}
$$

which is valid for any system, whether uniform or nonuniform, equilibrium or not [176-178]. Here,

$$
\rho(\mathbf{r})=\rho_{0}(\mathbf{r})+\rho_{1}(\mathbf{r})
$$

is the total particle density and

$$
g\left(\mathbf{r}, \mathbf{r}^{\prime}\right) \equiv \frac{\left\langle\hat{\psi}^{\dagger}(\mathbf{r}) \hat{\psi}^{\dagger}\left(\mathbf{r}^{\prime}\right) \hat{\psi}\left(\mathbf{r}^{\prime}\right) \hat{\psi}(\mathbf{r})\right\rangle}{\rho(\mathbf{r}) \rho\left(\mathbf{r}^{\prime}\right)}
$$

is the pair correlation function.

In the HFB approximation, analogously to the Bogolubov approximation, one has to retain in Eq. (551) the terms up to the second-order with respect to the operators of uncondensed particles. For nonuniform systems, one can employ the local-density approximation of Sec. 7.4. Then Eq. (551) reduces to

$$
\chi_{N}=1+\frac{2}{N} \int \rho(\mathbf{r}) \lim _{k \rightarrow 0}[n(\mathbf{k}, \mathbf{r})+\sigma(\mathbf{k}, \mathbf{r})] d \mathbf{r} .
$$

Using the formulas of Sec. 7.4 gives

$$
\chi_{N}=\frac{T}{m N} \int \frac{\rho(\mathbf{r})}{c^{2}(\mathbf{r})} d \mathbf{r} .
$$


The same Eq. (553) represents the structure factor (516). Equation (515), defining the hydrodynamic sound velocity, leads to

$$
s_{T}^{2}=\left[\frac{1}{N} \int \frac{\rho(\mathbf{r})}{c^{2}(\mathbf{r})} d \mathbf{r}\right]^{-1} .
$$

And the isothermal compressibility (514) becomes

$$
\kappa_{T}=\frac{1}{m \rho N} \int \frac{\rho(\mathbf{r})}{c^{2}(\mathbf{r})} d \mathbf{r},
$$

provided the average density $\rho$ is defined.

For a uniform system, the above formulas reduce to

$$
\chi_{N} \equiv \frac{\Delta^{2}(\hat{N})}{N}=S(0)=\frac{T}{m c^{2}}, \quad s_{T}=c, \quad \kappa_{T}=\frac{1}{m \rho c^{2}} .
$$

It is important to emphasize the necessity of taking into account the gauge symmetry breaking in the above calculations. If the symmetry would not be broken, or if the anomalous average $\sigma$ would be omitted, one would get the divergence of expressions (553) and (555), which would mean the system instability [179].

In some works on particle fluctuations, one also makes the following mistake. One writes that, in the canonical ensemble, the condensate fluctuations are given by $\Delta\left(\hat{N}_{0}\right)$ that is equal to $\Delta\left(\hat{N}_{1}\right)$, and one calculates the latter in the second quantization representation. However, this representation uses the field operators defined on the Fock space and, by construction, it is introduced for the grand canonical ensemble. So, $\Delta\left(\hat{N}_{1}\right)$ has nothing to do with condensate fluctuations that, by the Bogolubov theorem correspond to $\Delta\left(\hat{N}_{0}\right)=0$.

In this way, correct calculations lead to no anomalous thermodynamic particle fluctuations. The latter arise only in incorrect calculations. There are no anomalous fluctuations neither in correctly employed Bogolubov or HFB approximations [5,9,12,63,176-179] nor in the renormalization group approach [180].

If thermodynamically anomalous fluctuations would not be caused by calculational defects, but would be real, then not merely equilibrium Bose-condensed gas and superfluid helium would not exist, but the situation would be even more dramatic. This is because the systems with gauge symmetry $U(1)$ are just a particular case of systems with continuous symmetry, all such systems having general properties connected with their continuous symmetry and the symmetry breaking [181]. Therefore all such systems exhibiting thermodynamically anomalous fluctuations would not exist. We mean here only equilibrium statistical systems, since nonequilibrium systems can possess strong fluctuations making them unstable [182-184].

For example, many magnetic systems exhibit continuous symmetry connected with spin rotation. The appearance of magnetic order in such magnetic systems implies the spontaneous breaking of the spin rotational symmetry. If the continuous symmetry breaking would lead to the appearance of thermodynamically anomalous fluctuations of the order parameter, then, in magnetic systems, this would mean the occurrence of thermodynamically anomalous magnetic susceptibility, hence, instability. Then there would be no stable equilibrium magnetic systems with continuous symmetry breaking, which is again absurd.

To show that the spontaneous breaking of the spin-rotation symmetry does not lead to thermodynamically anomalous magnetic fluctuations [12], let us consider the Hesenberg model, with the Hamiltonian

$$
\hat{H}=-\sum_{i \neq j} J_{i j} \mathbf{S}_{i} \cdot \mathbf{S}_{j}-\sum_{i} \mathbf{B} \cdot \mathbf{S}_{i}
$$


in which $\mathbf{S}_{i}$ is a spin operator on the $i$-lattice site, $J_{i j}$ is an exchange interaction potential, and $\mathbf{B}$ is an external magnetic field. The Hamiltonian enjoys the spin rotation symmetry in the absence of the external field $\mathbf{B}$.

The Gibbs potential is defined as

$$
G=-T \ln \operatorname{Tr} e^{-\beta \hat{H}}=G(T, N, \mathbf{B})
$$

The system magnetic moment

$$
\mathbf{M} \equiv \frac{\partial G}{\partial \mathbf{B}}=-\left\langle\frac{\partial \hat{H}}{\partial \mathbf{B}}\right\rangle \equiv\langle\hat{\mathbf{M}}\rangle
$$

can be represented as the average of the magnetic-moment operator

$$
\hat{\mathbf{M}} \equiv-\frac{\partial \hat{H}}{\partial \mathbf{B}}=\sum_{i} \mathbf{S}_{i}
$$

The magnetic susceptibility tensor is given by the elements

$$
\chi_{\alpha \beta} \equiv \frac{1}{N} \frac{\partial M_{\beta}}{\partial B_{\alpha}}=-\frac{1}{N} \frac{\partial^{2} G}{\partial B_{\alpha} \partial B_{\beta}} .
$$

Direct calculations yield

$$
\chi_{\alpha \beta}=\frac{1}{N T} \operatorname{cov}\left(\hat{M}_{\alpha}, \hat{M}_{\beta}\right)
$$

which shows that the diagonal elements

$$
\chi_{\alpha \alpha}=\frac{\Delta^{2}\left(\hat{M}_{\alpha}\right)}{N T}
$$

are expressed through the dispersion of the components of the magnetic-moment operator (560).

The HFB approximation for the field operators of Bose systems is equivalent to the mean-field approximation for the spin operators of magnetic systems. Therefore, it is reasonable to resort here to the mean-field approximation, although the results are qualitatively the same if we invoke more elaborate techniques. In the mean-field approximation, Hamiltonian (557) reads as

$$
\hat{H}=-\sum_{i} \mathbf{H} \cdot \mathbf{S}_{i}+N J\left\langle\mathbf{S}_{i}\right\rangle^{2}
$$

in which the notation is used for the effective field

$$
\mathbf{H} \equiv 2 J\left\langle\mathbf{S}_{i}\right\rangle+\mathbf{B}
$$

and the effective interaction

$$
J \equiv \frac{1}{N} \sum_{i \neq j} J_{i j}
$$

For concreteness, let us consider spin one-half. Then the Gibbs potential (558) becomes

$$
G=-N T \ln \left[2 \cosh \left(\frac{H_{0}}{2 T}\right)\right]+N J\left\langle\mathbf{S}_{i}\right\rangle^{2},
$$


where the ideality of the lattice is implied and

$$
H_{0} \equiv|\mathbf{H}|=\sqrt{\sum_{\alpha} H_{\alpha}^{2}} .
$$

The average spin is defined by the extremization condition

$$
\frac{\partial G}{\partial\left\langle\mathbf{S}_{i}\right\rangle}=0
$$

which is equivalent to the equation

$$
\left\langle\mathbf{S}_{i}\right\rangle=-\frac{1}{N} \frac{\partial G}{\partial \mathbf{B}}
$$

As a result, one finds

$$
\left\langle\mathbf{S}_{i}\right\rangle=\frac{\mathbf{H}}{2 H_{0}} \tanh \left(\frac{H_{0}}{2 T}\right) .
$$

This defines the magnetic susceptibility (561) as

$$
\chi_{\alpha \beta}=\frac{\partial}{\partial B_{\alpha}}\left\langle S_{i}^{\beta}\right\rangle .
$$

Let us define the order parameter

$$
\eta \equiv 2\left|\left\langle\mathbf{S}_{i}\right\rangle\right|
$$

In view of Eq. (570), this reads as

$$
\eta=\tanh \left(\frac{H_{0}}{2 T}\right)
$$

Susceptibility (571) takes the form

$$
\begin{gathered}
\chi_{\alpha \beta}=\frac{\eta}{2 H_{0}}\left(\delta_{\alpha \beta}+2 J \chi_{\alpha \beta}\right)+ \\
+\frac{H_{\beta}}{2 H_{0}^{2}}\left(H_{\alpha}+2 J \sum_{\gamma} \chi_{\alpha \gamma} H_{\gamma}\right)\left(\frac{1-\eta^{2}}{2 T}-\frac{\eta}{H_{0}}\right) .
\end{gathered}
$$

Directing the external magnetic field along the axis $z$, so that

$$
B_{x}=B_{y}=0, \quad B_{z} \equiv h,
$$

yields

$$
H_{x}=H_{y}=0, \quad H_{z}=2 J\left\langle S_{i}^{z}\right\rangle+B_{z},
$$

which can be rewritten as

$$
H_{\alpha}=\delta_{\alpha z} H_{0}, \quad H_{0}=H_{z}=J \eta+h
$$

The average spin components become

$$
\left\langle S_{i}^{x}\right\rangle=\left\langle S_{i}^{y}\right\rangle=0, \quad\left\langle S_{i}^{z}\right\rangle=\frac{\eta}{2} .
$$


The susceptibility tensor (574) leads to the equation

$$
\chi_{\alpha \beta}=\frac{1}{2}\left(\delta_{\alpha \beta}+2 J \chi_{\alpha \beta}\right)\left[\frac{\eta}{H_{0}}+\delta_{\beta z}\left(\frac{1-\eta^{2}}{2 T}-\frac{\eta}{H_{0}}\right)\right],
$$

with the order parameter

$$
\eta=\tanh \left(\frac{J \eta+h}{2 T}\right) .
$$

Equation (578) shows that the nondiagonal elements are zero:

$$
\chi_{x y}=\chi_{x z}=\chi_{y z}=0
$$

while the diagonal elements give the transverse components

$$
\chi_{x x}=\chi_{y y}=\frac{\eta}{2 h}
$$

and the longitudinal component

$$
\chi_{z z}=\frac{1-\eta^{2}}{2\left[2 T-J\left(1-\eta^{2}\right)\right]} .
$$

The latter, with the notation for the critical temperature $T_{c} \equiv J / 2$, can be represented as

$$
\chi_{z z}=\frac{1-\eta^{2}}{4\left[T-T_{c}\left(1-\eta^{2}\right)\right]} .
$$

At low temperature, and $h \rightarrow 0$, the order parameter (579) behaves as

$$
\eta \simeq 1-2 \exp \left(-\frac{T_{c}}{T}\right) \quad\left(T \ll T_{c}\right)
$$

and susceptibility (583), as

$$
\chi_{z z} \simeq \frac{1}{T} \exp \left(-\frac{T_{c}}{T}\right) \quad\left(T \ll T_{c}\right) .
$$

At high temperature, and $h \rightarrow 0$, susceptibility (583) acquires the Curie-Weiss law

$$
\chi_{z z} \simeq \frac{1}{4\left(T-T_{c}\right)} \quad\left(T \geq T_{c}\right) .
$$

The latter susceptibility diverges at the critical point $T_{c}$. However, this divergence has nothing to do with the thermodynamically anomalous behavior, since this is the divergence with respect to temperature $T$, but not with respect to the number of particles $N$. In addition, the phase transition point is the point of system instability, where the system becomes nonequilibrium and fluctuations have right to infinitely rise.

One introduces the transverse susceptibility

$$
\chi_{\perp} \equiv \chi_{x x}=\frac{\Delta^{2}\left(\hat{M}_{x}\right)}{N T}
$$


and the longitudinal susceptibility

$$
\chi_{\|} \equiv \chi_{z z}=\frac{\Delta^{2}\left(\hat{M}_{z}\right)}{N T}
$$

where relation (563) is taken into account. In view of Eqs. (581) and (582), one finds the dispersions for the magnetic-moment operator (560), characterizing the transverse fluctuations,

$$
\frac{\Delta^{2}\left(\hat{M}_{x}\right)}{N}=T \chi_{\perp}=\frac{\eta T}{2 h}
$$

and the longitudinal fluctuations,

$$
\frac{\Delta^{2}\left(\hat{M}_{z}\right)}{N}=T \chi_{\|}=\frac{T\left(1-\eta^{2}\right)}{4\left[T-T_{c}\left(1-\eta^{2}\right)\right]},
$$

of the system magnetic moment.

The longitudinal fluctuations are always thermodynamically normal, if calculated in a selfconsistent way, as it should be, according to the stability condition (509). In some papers, one finds thermodynamically anomalous magnetic fluctuations of the same type as for Bose systems, with $\Delta\left(\hat{M}_{z}\right) / N \propto N^{1 / 3}$. But, as has been explained in Ref. [5], this is due to the same mistake as one does when dealing with Bose systems. One approximates Hamiltonian (557) by a secondorder form, with respect to small deviations from the average magnetic moment. And then, one considers the fourth-order form calculating the dispersion of $\hat{M}_{z}$. Going outside of the region of applicability of the chosen approximation leads to the appearance of meaningless results. But self-consistent calculations, as is shown above, always give normal longitudinal fluctuations.

The transverse fluctuations are known [185] to be much larger than the longitudinal ones. Formally, Eq. (589) diverges when $h \rightarrow 0$. This, however, does not make the transverse magnetic fluctuations thermodynamically anomalous. To be thermodynamically anomalous, expression (589) should diverge with respect to the number of particles $N$, or, what is the same, with respect to the system volume $V$. But here, it is the divergence with respect to $h$.

Moreover, one should not forget that below the transition temperature $T_{c}$ the spin-rotation symmetry is broken. The symmetry breaking is described by switching on a small external magnetic field $h \neq 0$. But then Eq. (589) is finite. Switching off this field restores the symmetry, as a result of which Eq. (589) would diverge, similarly to how the compressibility of a Bose-condensed system would diverge being incorrectly calculated without the gauge symmetry breaking. Therefore, as soon as the spin-rotation symmetry has been broken, when $h \neq 0$, all fluctuations are thermodynamically normal. And above $T_{c}$, where the symmetry is not broken, one has $\eta \cong h / 2 T$, hence $\Delta^{2}\left(\hat{M}_{x}\right) / N \cong 1 / 4$, which is again finite for any $h$.

When some symmetry in a system is broken, the mathematically correct definition of statistical averages is understood in the sense of the Bogolubov quasiaverages [16]. Then, as is well known, one has, first, to accomplish the thermodynamic limit, with $N \rightarrow \infty$ and, only after this, to consider the limit $h \rightarrow 0$. In that sense, there is no any thermodynamically anomalous fluctuations.

Note that real magnetic systems always possess magnetic anisotropy. This can be small, but never exactly zero, which corresponds to the presence of a finite $h$. Consequently, in real equilibrium magnetic systems, there are no thermodynamically anomalous fluctuations. And there are no thermodynamically anomalous fluctuations in any equilibrium system with the spontaneous breaking of any continuous symmetry. In the other case, such a system would be unstable and could not be in equilibrium. 


\section{Nonground-State Condensates}

\subsection{Coherent Modes}

First of all, it is necessary to concretize what is meant under nonground-state condensates. The stationary equation (335) for the condensate function can be treated as an eigenproblem. Generally, an eigenproblem can yield a spectrum of possible eigenvalues and a set of the related eigenfunctions. So, generally, the eigenproblem, corresponding to Eq. (335), can be represented in the form

$$
\begin{gathered}
{\left[-\frac{\nabla^{2}}{2 m}+U(\mathbf{r})\right] \eta_{n}(\mathbf{r})+} \\
+\Phi_{0}\left[\left|\eta_{n}(\mathbf{r})\right|^{2} \eta_{n}(\mathbf{r})+2 \rho_{1}(\mathbf{r}) \eta_{n}(\mathbf{r})+\sigma_{1}(\mathbf{r}) \eta_{n}^{*}(\mathbf{r})+\xi(\mathbf{r})\right]=E_{n} \eta_{n}(\mathbf{r}),
\end{gathered}
$$

in which the minimal eigenvalue defines the chemical potential

$$
\mu_{0}=\min _{n} E_{n}
$$

When $E_{n}=\mu_{0}$, Eq. (591) corresponds to the standard ground-state Bose-Einstein condensate, while, for higher eigenvalues $E_{n}$, this equation corresponds to nonground-state condensates. The values of $\rho_{1}(\mathbf{r}), \sigma_{1}(\mathbf{r})$, and $\xi(\mathbf{r})$ depend on the index $n$, but for short, this dependence is not shown explicitly.

The condensate function describes the coherent part of the system. In the limit of asymptotically weak interactions $\left(\Phi_{0} \rightarrow 0\right)$ and low temperature $(T \rightarrow 0)$, when the whole system is in the coherent state, Eq. (591) reduces to the nonlinear Scrödinger equation

$$
\left[-\frac{\nabla^{2}}{2 m}+U(\mathbf{r})\right] \eta_{n}(\mathbf{r})+\Phi_{0}\left|\eta_{n}(\mathbf{r})\right|^{2} \eta_{n}(\mathbf{r})=E_{n} \eta_{n}(\mathbf{r}) .
$$

In the particular case, for $E_{n}=\mu_{0}$, it is called the Gross-Pitaevskii equation.

The condensate function $\eta_{n}(\mathbf{r})$ is normalized to the number of condensed particles, as in Eq. (52). It is convenient to introduce the function $\varphi_{n}(\mathbf{r})$ by the relation

$$
\eta_{n}(\mathbf{r})=\sqrt{N_{0}} \varphi_{n}(\mathbf{r})
$$

so that $\varphi_{n}(\mathbf{r})$ be normalized to one,

$$
\int\left|\varphi_{n}(\mathbf{r})\right|^{2} d \mathbf{r}=1
$$

Then Eq. (591) transforms into

$$
\begin{gathered}
{\left[-\frac{\nabla^{2}}{2 m}+U(\mathbf{r})\right] \varphi_{n}(\mathbf{r})+} \\
+\Phi_{0}\left[N_{0}\left|\varphi_{n}(\mathbf{r})\right|^{2} \varphi_{n}(\mathbf{r})+2 \rho_{1}(\mathbf{r}) \varphi_{n}(\mathbf{r})+\sigma_{1}(\mathbf{r}) \varphi_{n}^{*}(\mathbf{r})+\frac{\xi(\mathbf{r})}{\sqrt{N_{0}}}\right]=E_{n} \varphi_{n}(\mathbf{r}),
\end{gathered}
$$

while Eq. (593), into

$$
\left[-\frac{\nabla^{2}}{2 m}+U(\mathbf{r})\right] \varphi_{n}(\mathbf{r})+\Phi_{0} N_{0}\left|\varphi_{n}(\mathbf{r})\right|^{2} \varphi_{n}(\mathbf{r})=E_{n} \varphi_{n}(\mathbf{r}) .
$$


The solutions to Eqs. (591) and (596) define the coherent modes in the general case [66] and Eqs. (593) and (597), the coherent modes for asymptotically weak interactions and temperature [186]. These coherent modes, first introduced in Ref. [186], correspond to nonground-state condensates. The properties of such coherent modes and the methods of their generation have been studied in a series of papers [66, 186-215]. A dipole coherent mode was excited in experiment [216]. These coherent modes are also called topological, since the nonground-state condensates, corresponding to different coherent modes, describe particle densities with different spatial topology.

\subsection{Trap Modulation}

There are several requirements that are necessary for creating a nonground-state condensate. First of all, it is clear that such a condensate cannot be equilibrium. Hence, its creation requires the action of external time-dependent fields. Second, the system of Bose particles has to possess a discrete spectrum in order that it would be possible to distinguish the usual ground-state BoseEinstein condensate from a nonground-state condensate. This means that the system is to be placed inside a trapping potential. And, third, to transfer particles from their ground-state to a chosen excited state, it is necessary, either to employ a resonant field or to use rather strong pumping.

A straightforward way of imposing external alternating fields is by modulating the trapping potential. Let the confining potential be composed of two parts,

$$
U(\mathbf{r}, t)=U(\mathbf{r})+V(\mathbf{r}, t)
$$

in which the first term is a trapping potential and the second term

$$
V(\mathbf{r}, t)=V_{1}(\mathbf{r}) \cos \omega t+V_{2}(\mathbf{r}) \sin \omega t
$$

realizes the modulation of this potential with frequency $\omega$.

There exists one limitation on the spatial dependence of the modulated trapping potential (598). In Refs. [206,207], the shape-conservation theorem has been proved, showing that the trap modulation moves the whole condensate without changing its shape if and only if the trapping potential $U(\mathbf{r})$ is harmonic, while the modulation term (599) is linear with respect to the spatial variables. In that case, the trap modulation would not be able to produce excited coherent modes. So, to generate these modes, one has to avoid this particular case of spatial dependence.

Suppose that at the initial time $t=0$ the system has been completely condensed, being in the energy state $E_{0}=\mu_{0}$. Then, to transfer the system to an energy state $E_{n}$, one has to use the alternating field with a frequency $\omega$ close to the transition frequency $\omega_{n}=E_{n}-\mu_{0}$. Under this resonance condition

$$
\left|\frac{\Delta \omega}{\omega}\right| \ll 1 \quad\left(\Delta \omega \equiv \omega-\omega_{n}\right),
$$

it is sufficient to invoke the pumping fields of small amplitudes.

The time-dependent equation (323) for the condensate function, after the substitution of the relation

$$
\eta(\mathbf{r}, t)=\sqrt{N_{0}} \varphi(\mathbf{r}, t)
$$

similar to Eq. (594), transforms into

$$
i \frac{\partial}{\partial t} \varphi(\mathbf{r}, t)=\left[-\frac{\nabla^{2}}{2 m}+U(\mathbf{r}, t)-\mu_{0}\right] \varphi(\mathbf{r}, t)+
$$




$$
+\Phi_{0}\left[N_{0} \rho_{0}(\mathbf{r}, t) \varphi(\mathbf{r}, t)+2 \rho_{1}(\mathbf{r}, t) \varphi(\mathbf{r}, t)+\sigma_{1}(\mathbf{r}, t) \varphi^{*}(\mathbf{r}, t)+\frac{\xi(\mathbf{r}, t)}{\sqrt{N_{0}}}\right] .
$$

We can look for the solution to this equation represented $[66,186,187,198]$ as an expansion over the coherent modes,

$$
\varphi(\mathbf{r}, t)=\sum_{n} C_{n}(t) \varphi_{n}(\mathbf{r}) e^{-i \omega_{n} t}
$$

so that the coefficient function $C_{n}(t)$ be slow as compared to the fast oscillating exponential functions:

$$
\frac{1}{\omega_{n}}\left|\frac{d C_{n}}{d t}\right| \ll 1 .
$$

Let us introduce the matrix elements corresponding to particle interactions,

$$
\alpha_{m n} \equiv \Phi_{0} N_{0} \int\left|\varphi_{m}(\mathbf{r})\right|^{2}\left[2\left|\varphi_{n}(\mathbf{r})\right|^{2}-\left|\varphi_{m}(\mathbf{r})\right|^{2}\right] d \mathbf{r}
$$

and to the action of the modulating field,

$$
\beta_{m n} \equiv \int \varphi_{m}^{*}(\mathbf{r})\left[V_{1}(\mathbf{r})-i V_{2}(\mathbf{r})\right] \varphi_{n}(\mathbf{r}) d \mathbf{r}
$$

Also, let us define the expression

$$
\begin{gathered}
\varepsilon_{n}(t) \equiv \alpha_{n n}- \\
-\Phi_{0} \int \varphi_{n}^{*}(\mathbf{r})\left[2 \rho_{1}^{(n)}(\mathbf{r}) \varphi_{n}(\mathbf{r})-2 \rho_{1}(\mathbf{r}, t) \varphi_{n}(\mathbf{r})+\sigma_{1}^{(n)}(\mathbf{r}) \varphi_{n}^{*}(\mathbf{r})+\frac{\xi^{(n)}(\mathbf{r})}{\sqrt{N_{0}}}\right] d \mathbf{r},
\end{gathered}
$$

in which the functions with the upper index $n$ correspond to the stationary solutions characterized by the condensate function $\varphi_{n}(\mathbf{r})$ and where

$$
\alpha_{n n}=\Phi_{0} N_{0} \int\left|\varphi_{n}(\mathbf{r})\right|^{4} d \mathbf{r} .
$$

The trap modulation produces not only the required coherent mode but it also destroys the condensate by transferring particles from the condensate to the fraction of uncondensed particles. Therefore, the generation of the coherent mode can be effectively done only during a finite depletion time $t_{d e p}$, when the transfer from the condensate to the uncondensed fraction is yet negligible. During this time, the variation of quantity (607) is small, such that

$$
\left|\frac{t}{\varepsilon_{n}} \frac{d \varepsilon_{n}}{d t}\right| \ll 1 \quad\left(t<t_{d e p}\right) .
$$

It is convenient to make the change

$$
C_{n}(t)=c_{n}(t) \exp \left[-i \varepsilon_{n}(t) t\right]
$$

in which $\varepsilon_{n}=\varepsilon_{n}(t)$ is treated as a slow function of time, in the sense of inequality (608). We may notice that in the limit of a completely coherent system, when the fraction of uncondensed particles is negligibly small, Eq. (607) does not depend on time.

Then, we substitute expansion (603) into Eq. (602), employ the above notations, and invoke the averaging techniques [114,117,119,121,217-219], based on the existence of different time scales 
[220,221]. As initial conditions, we assume nonzero $c_{n}(0)$ and $c_{0}(0)$, while all other coefficient functions $c_{j}(0)=0$ for $j \neq 0, n$. This procedure yields $[66,186,195]$ the equations

$$
\begin{gathered}
i \frac{d c_{0}}{d t}=\alpha_{0 n}\left|c_{n}\right|^{2} c_{0}+\frac{1}{2} \beta_{0 n} c_{n} e^{i \Delta \omega t}, \\
i \frac{d c_{n}}{d t}=\alpha_{n 0}\left|c_{0}\right|^{2} c_{n}+\frac{1}{2} \beta_{0 n}^{*} c_{0} e^{-i \Delta \omega t} .
\end{gathered}
$$

Solving these equations gives the fractional mode populations

$$
p_{n}(t) \equiv\left|c_{n}(t)\right|^{2}
$$

As a concluding remark to this section, it is worth emphasizing that expansion (603) corresponds to the diabatic representation [66,215], and one should not confuse it with the adiabatic representation [222], which is not suitable for the studied resonant process.

\subsection{Interaction Modulation}

Another way of exciting the cloud of particles confined inside a trap is by varying the particle interactions by means of the Feshbach-resonance techniques [1-3,5,34,223]. This method can also be used for generating the coherent modes, as has been mentioned in Refs. [206,207,210], and analyzed in detail in Refs. [214,215].

Let the scattering length be modulated so that the particle interaction becomes time-dependent according to the law

$$
\Phi(t)=\Phi_{0}+\Phi_{1} \cos (\omega t)+\Phi_{2} \sin (\omega t) .
$$

Following the same procedure as in the case of the trap modulation and introducing the notation

$$
\gamma_{m} \equiv N_{0}\left(\Phi_{1}-i \Phi_{2}\right) \int \varphi_{0}^{*}(\mathbf{r})\left|\varphi_{m}(\mathbf{r})\right|^{2} \varphi_{n}(\mathbf{r}) d \mathbf{r}
$$

in which $n$ is fixed and $m=0, n$, we get the equations

$$
\begin{aligned}
& i \frac{d c_{0}}{d t}=\alpha_{0 n}\left|c_{n}\right|^{2} c_{0}+\left(\gamma_{0}\left|c_{0}\right|^{2}+\frac{1}{2} \gamma_{n}\left|c_{n}\right|^{2}\right) c_{n} e^{i \Delta \omega t}+\frac{1}{2} \gamma_{0}^{*} c_{n}^{*} c_{0}^{2} e^{-i \Delta \omega t}, \\
& i \frac{d c_{n}}{d t}=\alpha_{n 0}\left|c_{0}\right|^{2} c_{n}+\left(\gamma_{n}^{*}\left|c_{n}\right|^{2}+\frac{1}{2} \gamma_{0}^{*}\left|c_{0}\right|^{2}\right) c_{0} e^{-i \Delta \omega t}+\frac{1}{2} \gamma_{n} c_{0}^{*} c_{n}^{2} e^{i \Delta \omega t} .
\end{aligned}
$$

Both these ways of modulating either the trapping potential or particle interactions can be used for generating excited coherent modes.

Nonequilibrium systems with the generated coherent modes, representing nonground-state condensates, possess a variety of interesting properties. We can mention the following effects: interference patterns and interference currents [194,195,198], mode locking [186,198,199], dynamical phase transitions and critical phenomena [190,194,195,198], chaotic motion [206,207], atomic squeezing [198,201,202], Ramsey fringes [211-213], and entanglement production [224-226] that can be quantified by a general measure of entanglement production [227-229].

The above-mentioned effects can be realized by resonant alternating fields of rather low amplitudes. When increasing the amplitude of the pumping field, it becomes feasible to generate the excited coherent modes with the frequencies of the alternating fields, which are not exactly in resonance with the transition frequencies. Thus, the transition between the coherent modes, 
characterized by the transition frequency $\omega_{12}$, can be done by means of the harmonic generation and parametric conversion [206,207].

Harmonic generation occurs, when the driving frequency $\omega$ satisfies the condition

$$
n \omega=\omega_{12} \quad(n=1,2, \ldots) .
$$

Parametric conversion requires the use of two alternating fields, with the driving frequencies $\omega_{1}$ and $\omega_{2}$, such that

$$
\omega_{1} \pm \omega_{2}=\omega_{12}
$$

In the case of two pumping fields, there exists the combined resonance under the condition

$$
n_{1} \omega_{1}+n_{2} \omega_{2}=\omega_{12} \quad\left(n_{i}= \pm 1, \pm 2, \ldots\right) .
$$

And, generally, the application of several external alternating fields, with the driving frequencies $\omega_{i}$, can generate coherent modes under the condition of generalized resonance, when

$$
\sum_{i} n_{i} \omega_{i}=\omega_{12} \quad\left(n_{i}= \pm 1, \pm 2, \ldots\right)
$$

The amplitudes of external alternating fields can be made arbitrarily strong. Therefore, all these effects can be realized in experiments. The particle interactions can also be varied in a wide range. For instance, employing the Feshbach resonance techniques, it is possible to tune the interactions of ${ }^{7} \mathrm{Li}$ atoms over seven orders of magnitude [230]. Hence, the generation of nonground-state condensates can be done by the interaction modulation as well.

\subsection{Turbulent Superfluid}

As follows from the previous sections, increasing the modulation amplitude results in the generation of more and more coherent modes, whose excitation becomes more and more easy, especially when several alternating fields are involved. This is because it is sufficient that the frequencies of the modulating fields be such that one of the above conditions be approximately satisfied. As soon as this happens, the related coherent modes become excited. Intensive field modulation generates simultaneously several coherent modes.

When alternating fields are applied creating an oscillating anisotropy, with local rotation moments, then the prevailing coherent modes will be quantum vortices. An important feature of the vortex creation by means of the anisotropic trap modulation, contrary to the vortex creation by means of rotation, is the generation of vortices as well as antivortices, that is, the generation of the vortices with opposite rotation velocities. The oppositely rotating vortices repel each other and diffuse in space, separating from each other. At the beginning, when the amplitude modulation is not yet too strong, there should arise just a small number of vortices having the standard properties [1-3,231,232], except that vortices and antivortices both are present. In the case, when the whole system is uniformly rotated, the increased rotation frequency induces a vortex lattice $[231,232]$. Contrary to this, when the trapped system is subject to the action of alternating fields, nonuniformly and anisotropically shaking the trapped particle cloud, the created vortices possess different axes of rotation and different rotation velocities. Therefore no vortex lattice is possible. Then, increasing the amplitude of the alternating fields produces a large number of vortices with randomly distributed vorticities. Such a tangle of quantized vortices forms what is called quantum turbulence, and the whole system is said to be in the state of turbulent superfluid. 
The problem of turbulent superfluid has been addressed in a number of works. The related literature has been reviewed in articles [233-237] (see also recent Refs. [238-240]). There are plenty of experiments observing quantum turbulence in liquid ${ }^{3} \mathrm{He}$ and ${ }^{4} \mathrm{He}$. Quantum turbulence in trapped gases has also been observed [241]. The description of turbulent superfluids as continuous vortex mixtures has been advanced [237].

\subsection{Heterophase Fluid}

Increasing further the amplitude of the alternating field breaks the turbulent superfluid into spatially separated pieces, with Bose-condensed droplets separated by normal, nonsuperfluid, spatial regions. Such a state reminds the Bose glass, or granular condensate, considered in Sec. 9.5. But now it is a highly nonequilibrium state. This state is analogous to heterophase mixtures consisting of several randomly intermixed phases [54]. Thence, it is called heterophase fluid.

An external modulating field acts on the system similarly to the action of a spatial random potential [110], such as treated in Sec. 9. The possibility of mapping the system with a timedependent modulation to the system with a spatially random potential is very important, since it allows us to understand the behavior of modulated nonequilibrium systems by comparing them with equilibrium random systems. The proof of this mapping is as follows.

Let the system Hamiltonian

$$
H(t)=H_{0}+\hat{V}(t)
$$

consist of the usual term $H_{0}$, containing no time-dependent fields, and a term

$$
\hat{V}(t)=\int \hat{\psi}^{\dagger}(\mathbf{r}) V(\mathbf{r}, t) \hat{\psi}(\mathbf{r}) d \mathbf{r},
$$

with an external potential depending on time. The characteristic variation time $t_{m o d}$ of the modulating potential $V(\mathbf{r}, t)$ is assumed to be much longer than the local-equilibrium time $t_{l o c}$, but much shorter than the time of experiment $t_{\text {exp }}$,

$$
t_{l o c} \ll t_{\text {mod }} \ll t_{\text {exp }} .
$$

The modulating potential pumps energy into the system that can be associated with the effective temperature

$$
T^{*} \equiv \frac{1}{N} \int_{0}^{t_{\exp }}\left|\left\langle\frac{\partial \hat{V}(t)}{\partial t}\right\rangle\right| d t
$$

If the pumping potential is periodically oscillating with a frequency $\omega$ and period $t_{m o d}=2 \pi / \omega$, then

$$
\frac{\partial \hat{V}(t)}{\partial t}=\omega \hat{V}(t)=\frac{2 \pi}{t_{\text {mod }}} \hat{V}(t) .
$$

In this case, the effective temperature (622) is

$$
T^{*}=\frac{2 \pi}{N t_{m o d}} \int_{0}^{t_{\text {exp }}}|\langle\hat{V}(t)\rangle| d t .
$$

Denoting the amplitude of the modulating potential $V(\mathbf{r}, t)$ as $V_{\text {mod }}$, we have

$$
|\langle\hat{V}(t)\rangle| \approx N V_{\text {mod }}
$$


Therefore, the effective temperature (623) becomes

$$
T^{*} \approx 2 \pi \frac{t_{\text {exp }}}{t_{\text {mod }}} V_{\text {mod }} .
$$

One may notice that the effective temperature depends on $t_{\text {exp }}$, though this dependence is week, in the sense that

$$
\frac{t_{m o d}}{T^{*}}\left|\frac{\partial T^{*}}{\partial t_{e x p}}\right| \leq \frac{t_{m o d}}{t_{e x p}} \ll 1 .
$$

Under the slow modulation, such that $t_{m o d} \gg t_{l o c}$, the system, at each moment of time, is in quasiequilibrium. Consequently, one can define the local in time thermodynamic potential

$$
\Omega(t)=-T^{*} \ln \operatorname{Tr} \exp \left\{-\beta^{*} H(t)\right\},
$$

where $\beta^{*}=1 / T^{*}$. Because $t_{\text {exp }} \gg t_{m o d}$, we are interested not in the local potential (625) but in the coarse-grained potential

$$
\Omega=\frac{1}{t_{m o d}} \int_{0}^{t_{m o d}} \Omega(t) d t,
$$

averaged over oscillations that are fast as compared to $t_{\text {exp }}$.

At each moment of time $t$ the potential $V(\mathbf{r}, t)$ describes a spatial potential. This can be characterized by the relation

$$
V(\mathbf{r}, t)=\xi(\mathbf{r}),
$$

which defines the functional

$$
t=t[\xi(\mathbf{r})] .
$$

Equations (627) and (628) symbolize the fact that for each time $t$ there corresponds a spatial potential $\xi(\mathbf{r})$ and vice versa, a potential $\xi(\mathbf{r})$ is ascribed to time $t$. The relation between the interval $\left[0, t_{m o d}\right]$ and the topological space $\{\xi(\mathbf{r})\}$, without much loss of generality, can be taken as homeomorphic.

The variation of time is equivalent to the variation of the spatial potential, so that

$$
d t=\frac{\delta t[\xi(\mathbf{r})]}{\delta \xi(\mathbf{r})} \delta \xi(\mathbf{r}) .
$$

With relation (628), Hamiltonian (619) becomes the functional

$$
H[\xi(\mathbf{r})]=H_{0}+\int \hat{\psi}^{\dagger}(\mathbf{r}) \xi(\mathbf{r}) \hat{\psi}(\mathbf{r}) d \mathbf{r}
$$

of the spatial field $\xi(\mathbf{r})$. Therefore, the averaged thermodynamic potential (626) takes the form

$$
\Omega=-T^{*} \int \ln \operatorname{Tr} \exp \left\{-\beta^{*} H[\xi(\mathbf{r})]\right\} \mathcal{D} \xi(\mathbf{r}) .
$$

The latter is equivalent to the thermodynamic potential of an equilibrium system in a random external field.

If the external alternating field has an amplitude $V_{\bmod }$ and the whole trapped system is subject to the modulation, then the modulation amplitude $V_{\text {mod }}$ plays the role of the correlation amplitude $V_{R}$ and the effective trap length $l_{0}$, of the correlation length $l_{R}$ in Eq. (459). The effective trap length $l_{0}=1 / \sqrt{m \omega_{0}}$ and the effective trap frequency $\omega_{0}$ are defined in Eq. (532). 
More strictly, the system state, produced by the trap modulation, depends not merely on the modulation amplitude $V_{\text {mod }}$, but on the amount of the total energy pumped into the system, playing the role of the effective temperature in the nonequilibrium system [237]. For an alternating field, with the driving frequency $\omega$ and the related period $t_{\text {mod }}=2 \pi / \omega$, acting on the system during the total time of experiment $t_{\text {exp }}$, the pumped energy is associated with the effective temperature (624). This energy should be treated as the effective modulation amplitude. Thus, instead of the localization length (460), we get

$$
l_{l o c}=\frac{\hbar^{4}}{m^{2} V_{0}^{2} l_{0}^{3}}=\left(\frac{\hbar \omega_{0}}{V_{0}}\right)^{2} l_{0},
$$

where, for clarity, the Planck constant is restored.

Depending on the relation between the localization length (631) and the trap length $l_{0}$, there can exist the following states:

$$
\begin{array}{ll}
l_{\text {loc }}>l_{0} & (\text { superfluid) }, \\
a<l_{l o c}<l_{0} & \text { (heterophase fluid) } \\
l_{l o c} \leq a & (\text { chaotic fluid) } .
\end{array}
$$

The superfluid state here includes all types of superfluids, the regular superfluid having no vortices, the vortex superfluid with a small number of vortices, and the turbulent superfluid with a random tangle of many vortices. This classification, in terms of the pumped energy, reads as follows:

$$
\begin{array}{ll}
V_{0}<\hbar \omega_{0} & (\text { superfluid) }, \\
\hbar \omega_{0}<V_{0}<\hbar \omega_{0} \sqrt{l_{0} / a} & \text { (heterophase fluid) } \\
V_{0} \geq \hbar \omega_{0} \sqrt{l_{0} / a} & \text { (chaotic fluid) } .
\end{array}
$$

Chaotic fluid is a strongly fluctuating system having neither long-range order nor even local order. It resembles the state of weak turbulence [242] or the chaotic state [243]. Qualitatively, the overall scheme, representing the sequence of states arising under the action of an alternating field, with respect to the amount of the pumped energy $V_{0}$, is shown [237] in Figure 1.

After the external modulation field is switched off, a finite quantum system relaxes to its equilibrium state during the relaxation time defined by particle collisions, the trap size, and trap shape [244]. The relaxation time becomes quite long for quasi-one-dimensional traps, where it may last, without equilibration for thousands of collisions between the oscillating Bose-condensed droplets [245]. This is because the one-dimensional system with local interactions is the integrable Lieb-Liniger system [246,247]. And quasi-one-dimensional systems, approaching integrability, display very long equilibration times.

In a quasi-one-dimensional trap, collisions, restricted to the motion of particles in the axial direction, with the particles remaining in the same transverse ground state, are not accompanied by energy change, hence, do not lead to thermalization. For such two-body collisions, equilibration and thermalization occurs only under transverse excitations. The corresponding rate of populating the radially excited modes by pairwise collisions, can be estimated from the Fermi golden rule that, at low temperature $T<\omega_{\perp}$, gives $[248,249]$ the rate

$$
\Gamma_{2} \approx 2.8 \omega_{\perp} \zeta \exp \left(-2 \omega_{\perp} / T\right)
$$

where the dimensionless parameter

$$
\zeta \equiv \rho_{1 d} \frac{a_{s}^{2}}{l_{\perp}}
$$


is expressed through the three-dimensional scattering length $a_{s}$, transverse oscillator length $l_{\perp}$, and the one-dimensional density

$$
\rho_{1 d}=\rho \pi l_{\perp}^{2}=\frac{N}{2 l_{z}} .
$$

At temperature tending to zero, this rate is exponentially suppressed. However, it can be essential for finite temperatures.

For quasi-one-dimensional traps at low temperatures, the three-body collision rate [249] can become important,

$$
\Gamma_{3} \approx 6.9 \zeta^{2} \omega_{\perp}
$$

The states, described above, have been experimentally realized by means of the trap modulation [241,250]. The whole diagram, showing the dependence of the produced states on the modulation amplitude and modulation time has been presented [250], starting from the regular superfluid, through vortex superfluid, to turbulent superfluid, and to heterophase fluid.

\section{Conclusions}

In this review, the basic theoretical problems have been considered, arising in the description of systems with Bose-Einstein condensate. The solutions to these problems are elucidated. The main conclusions can be briefly summarized as follows.

(i) The global gauge symmetry breaking is the necessary and sufficient condition for BoseEinstein condensation. This is an exact mathematical fact. The symmetry breaking results in the appearance of both, the condensate fraction and anomalous averages. The latter cannot be neglected without destroying the theory self-consistency. Omitting the anomalous averages is principally wrong, yielding unreliable and often unreasonable results.

(ii) The Hohenberg-Martin dilemma of conserving versus gapless theories is resolved by introducing two Lagrange multipliers guaranteeing the validity of two normalization conditions, for the numbers of condensed and uncondensed particles. The use of these two Lagrange multipliers is necessary as soon as the global gauge symmetry has been broken.

(iii) Bose-Einstein condensed systems in strong spatially random potentials can be described by means of the method of stochastic decoupling. Perturbation theory with respect to the strength of disorder can fail, leading to incorrect conclusions.

(iv) Thermodynamically anomalous fluctuations of any observable quantities are strictly prohibited in all equilibrium statistical systems, irrespectively of the used representative statistical ensemble. Thermodynamically anomalous particle fluctuations, of either condensed or uncondensed particles, cannot exist in Bose-condensed systems. The occurrence of thermodynamically anomalous fluctuations can be due only to calculational mistakes.

(v) The method has been suggested of generating nonground-state condensates of trapped particles. The method can be realized by applying alternating external fields modulating either the trapping potential or particle interactions. This makes it possible to create different types on nonground-state condensates, such as coherent modes, turbulent superfluids, and heterophase fluids.

\section{Acknowledgments}

I am very much grateful for many useful discussions and permanent collaboration to V.S. Bagnato and E.P. Yukalova. Financial support from the Russian Foundation for Basic Research is appreciated. 


\section{References}

[1] L. Pitaevskii and S. Stringari, Bose-Einstein Condensation (Clarendon, Oxford, 2003).

[2] C.J. Pethik and H. Smith, Bose-Einstein Condensation in Dilute Gases (Cambridge University, Cambridge, 2008).

[3] P.W. Courteille, V.S. Bagnato, and V.I. Yukalov, Laser Phys. 11, 659 (2001).

[4] J.O. Andersen, Rev. Mod. Phys. 76, 599 (2004).

[5] V.I. Yukalov, Laser Phys. Lett. 1, 435 (2004).

[6] K. Bongs and K. Sengstock, Rep. Prog. Phys. 67, 907 (2004).

[7] V.I. Yukalov and M.D. Girardeau, Laser Phys. Lett. 2, 375 (2005).

[8] A. Posazhennikova, Rev. Mod. Phys. 78, 1111 (2006).

[9] V.I. Yukalov, Laser Phys. Lett. 4, 632 (2007).

[10] N.P. Proukakis and B. Jackson, J. Phys. B 41, 203002 (2008).

[11] C. Moseley, O. Fialko, and K. Ziegler, Ann. Physik 17, 561 (2008).

[12] V.I. Yukalov, Laser Phys. 19, 1 (2009).

[13] N.N. Bogolubov, J. Phys. (Moscow) 11, 23 (1947).

[14] N.N. Bogolubov, Moscow Univ. Phys. Bull. 7, 43 (1947).

[15] N.N. Bogolubov, Lectures on Quantum Statistics (Gordon and Breach, New York, 1967), Vol. 1.

[16] N.N. Bogolubov, Lectures on Quantum Statistics (Gordon and Breach, New York, 1970), Vol. 2.

[17] E.P. Gross, Phys. Rev. 106, 161 (1957).

[18] E.P. Gross, Ann. Phys. (N.Y.) 4, 57 (1958).

[19] E.P. Gross, Nuovo Cimento 20, 454 (1961).

[20] V.L. Ginzburg and L.P. Pitaevskii, J. Exp. Theor. Phys. 7, 858 (1958).

[21] L.P. Pitaevskii, J. Exp. Theor. Phys. 13, 451 (1961).

[22] V. Letokhov, Laser Control of Atoms amd Molecules (Oxford University, New York, 2007).

[23] F. London, Superfluids: Microscopic Theory of Superfluid Helium, (Dover, New York, 1954), Vol. 2.

[24] F.H. Wirth and R.B. Hallock, Phys. Rev. B 35, 89 (1987).

[25] F. Cummings, G. Hyland, and G. Rowlands, Phys. Lett. A 86, 370 (1981). 
[26] H. Ghassib and R. Sridhar, Phys. Lett. A 100, 198 (1984).

[27] W.K. Chow, K.W. Wong, and P.C. Fung, J. Phys. Soc. Jap. 54, 4490 (1985).

[28] V.I. Yukalov, Theor. Math. Phys. 37, 1093 (1978).

[29] V.I. Yukalov, Physica A 100, 431 (1980).

[30] V.I. Yukalov, Phys. Lett. A 83, 26 (1981).

[31] V.I. Yukalov, Physica B 107, 233 (1981).

[32] L.D. Landau, Collected Papers (Gordon and Breach, New York, 1967).

[33] T. Köhler, K. Goral, and P.S. Julienne, Rev. Mod. Phys. 78, 1311 (2006).

[34] V.A. Yurovsky, M. Olshanii, and D.S. Weiss, Adv. At. Mol. Opt. Phys. 55, 61 (2008).

[35] M. Girardeau and R. Arnowitt, Phys. Rev. 113, 755 (1959).

[36] M. Girardeau, J. Math. Phys. 3, 131 (1962).

[37] N.M. Hugenholtz and D. Pines, Phys. Rev. 116, 489 (1959).

[38] P.C. Hohenberg and P.C. Martin, Ann. Phys. (N.Y.) 34, 291 (1965).

[39] V.N. Popov, Functional Integrals in Quantum Field Theory and Statistical Physics (Reidel, Dordrecht, 1983).

[40] V.N. Popov, Functional Integrals and Collective Modes (Cambridge University, New York, 1987).

[41] C.N. Yang, Rev. Mod. Phys. 34, 694 (1962).

[42] A.J. Coleman and V.I. Yukalov, Reduced Density Matrices (Springer, Berlin, 2000).

[43] O. Penrose and L. Onsager, Phys. Rev. 104, 576 (1956).

[44] A.J. Coleman and V.I. Yukalov, Mod. Phys. Lett. B 5, 1679 (1991).

[45] A.J. Coleman and V.I. Yukalov, Nuovo Cimento B 107, 535 (1992).

[46] A.J. Coleman and V.I. Yukalov, Nuovo Cimento B 108, 1377 (1993).

[47] A.J. Coleman and V.I. Yukalov, Int. J. Mod. Phys. B 10, 3505 (1996).

[48] V.I. Yukalov, Physica A 310, 413 (2002).

[49] T.L. Hill, Statistical Mechanics (McGraw-Hill, New York, 1956).

[50] A. Münster, Chemical Thermodynamics (Akademie, Berlin, 1969).

[51] V.I. Yukalov, Phys. Rev. A 72, 033608 (2005).

[52] E.H. Lieb, Bull. Am. Math. Soc. 22, 1 (1990). 
[53] V.I. Yukalov, Int. J. Mod. Phys. B 5, 3235 (1991).

[54] V.I. Yukalov, Phys. Rep. 208, 395 (1991).

[55] J. Ginibre, Commun. Math. Phys. 8, 26 (1968).

[56] G. Roepstorff, J. Stat. Phys. 18, 191 (1978).

[57] A. Süto, Phys. Rev. A 71, 023602 (2005).

[58] E.H. Lieb, R. Seiringer, J.P. Solovej, and J. Yngvason, The Mathematics of the Bose Gas and Its Condensation (Birkhauser, Basel, 2005).

[59] J.W. Gibbs, Collected Works (Longmans, New York, 1928), Vol. 1.

[60] J.W. Gibbs, Collected Works (Longmans, New York, 1931), Vol. 2.

[61] D. Ter Haar, Rev. Mod. Phys. 27, 289 (1955).

[62] D. Ter Haar and H. Wergeland, Elements of Thermodynamics (Addison-Wesley, Reading, 1967).

[63] V.I. Yukalov, Phys. Rev. E 72, 066119 (2005).

[64] V.I. Yukalov, Int. J. Mod. Phys. B 21, 69 (2007).

[65] V.I. Yukalov, Laser Phys. 16, 511 (2006).

[66] V.I. Yukalov, Laser Phys. Lett. 3, 406 (2006).

[67] V.I. Yukalov, Phys. Lett. A 359, 712 (2006).

[68] V.I. Yukalov and H. Kleinert, Phys. Rev. A 73, 063612 (2006).

[69] V.I. Yukalov and E.P. Yukalova, Phys. Rev. A 74, 063623 (2006).

[70] V.I. Yukalov and E.P. Yukalova, Phys. Rev. A 76, 013602 (2007).

[71] V.I. Yukalov, Ann. Phys. (N.Y.) 323, 461 (2008).

[72] M.K. Prasad, Physica D 1, 167 (1980).

[73] H. Kleinert, Path Integrals (World Scientific, Singapore, 2006).

[74] J.S. Langer, Ann. Phys. (N.Y.) 54, 258 (1969).

[75] E.M. Lifshitz and L.P. Pitaevskii, Physical Kinetics (Pergamon, Oxford, 1981).

[76] V.I. Yukalov, Laser Phys. Lett. 3, 106 (2006).

[77] L.D. Landau and E.M. Lifshitz, Mechanics (Pergamon, Oxford, 1960).

[78] L.D. Landau and E.M. Lifshitz, Fluid Mechanics (Pergamon, Oxford, 1987).

[79] J.E. Mayer and M.E Mayer, Statistical Mechanics (Wiley, New York, 1977). 
[80] F. Schlögl, Phys. Rep. 62, 267 (1980).

[81] V.I. Yukalov, Statistical Green's Functions (Queen's University, Kingston, 1998).

[82] V.L. Bonch-Bruevich and S.V. Tyablikov, Green Function Method in Statistical Mechanics (Interscience, New York, 1962).

[83] V.I. Yukalov, Theor. Math. Phys. 17, 1244 (1973).

[84] N.N. Bogolubov and N.N. Bogolubov Jr., Introduction to Quantum Statistical Mechanics (Gordon and Breach, Lausanne, 1994).

[85] A.D. Haymet, Prog. Solid State Chem. 17, 1 (1986).

[86] L.D. Landau and E.M. Lifshitz, Quantum Mechanics (Butterworth-Heinemann, Amsterdam, 2003).

[87] F.F. Abraham, Phys. Rep. 53, 93 (1979).

[88] R.C. Tolman, Relativity, Thermodynamics, Cosmology (Clarendon, Oxford, 1969).

[89] D.N. Zubarev Nonequilibrium Statistical Thermodynamics (Consultants Bureau, New York, 1974).

[90] D. Ruelle, Statistical Mechanics (Benjamin, New York, 1969).

[91] V.I. Yukalov and E.P. Yukalova, Phys. Rev. A 72, 063611 (2005).

[92] J. van Wezel, J. van den Brink, and J. Zaanen, Phys. Rev. Lett. 94, 230401 (2005).

[93] J. van Wezel, J. Zaanen, and J. van den Brink, Phys. Rev. B 74, 094430 (2006).

[94] J. van Wezel and J. van den Brink, Am. J. Phys. 75, 635 (2007).

[95] J. van Wezel and J. van den Brink, Phys. Rev. B 77, 064523 (2008).

[96] T.D. Lee and C.N. Yang, Phys. Rev. 105, 1119 (1957).

[97] T.D. Lee, K. Huang, and C.N. Yang, Phys. Rev. 106, 1135 (1957).

[98] T.D. Lee and C.N. Yang, Phys. Rev. 112, 1419 (1958).

[99] L. Pollet, N.V. Prokofev, B.V. Svistunov, and M. Troyer, Phys. Rev. Lett. 103, 140402 (2009).

[100] V. Gurarie, L. Pollet, N.V. Prokofev, B.V. Svistunov, and M. Troyer, Phys. Rev. B 80, 214519 (2009).

[101] Y. Hu, Z. Liang, and B. Hu, arXiv:1004.3333 (2010).

[102] K. Huang and H.F. Meng, Phys. Rev. Lett. 69, 644 (1992).

[103] A.V. Lopatin and V.M. Vinokur, Phys. Rev. Lett. 88, 235503 (2002).

[104] T. Nattermann and V.L. Pokrovsky, Phys. Rev. Lett. 100, 060402 (2008). 
[105] C. Gaul, N. Renner, and C.A. Müller, Phys. Rev. A 80, 053620 (2009).

[106] O. Zobay, Laser Phys. 19, 700 (2009).

[107] S. Pilati, S. Giorgini, and N. Prokofev, Phys. Rev. Lett. 102, 150402 (2009).

[108] V.I. Yukalov and R. Graham, Phys. Rev. A 75, 023619 (2007).

[109] V.I. Yukalov, E.P. Yukalova, K.V. Krutitsky, and R. Graham, Phys. Rev. A 76, 053623 (2007).

[110] V.I. Yukalov, E.P. Yukalova, and V.S. Bagnato, Laser Phys. 19, 686 (2009).

[111] N.G. Van Kampen, Stochastic Processes in Physics and Chemistry (North-Holland, Amsterdam, 1981).

[112] V.I. Yukalov, Laser Phys. 8, 1182 (1998).

[113] V.I. Yukalov, Opt. Spectrosc. 87, 550 (1999).

[114] V.I. Yukalov and E.P. Yukalova, Phys. Part. Nucl. 31, 561 (2000).

[115] V.I. Yukalov, Proc. SPIE 4605, 237 (2001).

[116] V.I. Yukalov, Laser Phys. 5, 526 (1995).

[117] V.I. Yukalov, Laser Phys. 5, 970 (1995).

[118] V.I. Yukalov, Phys. Rev. Lett. 75, 3000 (1995).

[119] V.I. Yukalov, Phys. Rev. B 53, 9232 (1996).

[120] V.I. Yukalov, Laser Phys. 12, 1089 (2002).

[121] V.I. Yukalov and E.P. Yukalova, Phys. Part. Nucl. 35, 348 (2004).

[122] S. Giorgini, L. Pitaevskii, and S. Stringari, Phys. Rev. B 49, 12938 (1994).

[123] V.I. Yukalov, Phys. Rev. A 42, 3324 (1990).

[124] V.I. Yukalov, Physica A 167, 833 (1990).

[125] V.I. Yukalov, J. Math. Phys. 32, 1235 (1991).

[126] V.I. Yukalov, J. Math. Phys. 33, 3994 (1992).

[127] V.I. Yukalov and E.P. Yukalova, Physica A 206, 553 (1994).

[128] V.I. Yukalov and E.P. Yukalova, Physica A 225, 336 (1996).

[129] V.I. Yukalov and E.P. Yukalova, Ann. Phys. (N.Y.) 277, 219 (1999).

[130] V.I. Yukalov, S. Gluzman, and D. Sornette, Physica A 328, 409 (2003).

[131] S. Gluzman, V.I. Yukalov, and D. Sornette, Phys. Rev. E 67, 026109 (2003). 
[132] V.I. Yukalov and E.P. Yukalova, Phys. Lett. A 368, 341 (2007).

[133] B.B. Mandelbrot, The Fractal Geometry of Nature (Freeman, New York, 1983).

[134] P. Coleman and L. Pietronero, Phys. Rep. 213, 311 (1992).

[135] J.W. Lynn, Phys. Rev. B 11, 2624 (1975).

[136] J.W. Lynn and H.A. Mook, Phys. Rev. B 23, 198 (1981).

[137] C.J. Maetz, U. Gerhardt, E. Dietz, A. Ziegler, and R.J. Jelitto, Phys. Rev. Lett. 48, 1686 (1982).

[138] H.A. Mook and J.W. Lynn, J. Appl. Phys. 57, 3006 (1985).

[139] X. Tao, D.P. Landau, T.C. Schulthess, and G.M. Stocks, Phys. Rev. Lett. 95, 087207 (2005).

[140] A. Rigamonti, Adv. Phys. 33, 115 (1984).

[141] V.I. Yukalov, Ferroelectrics 82, 11 (1988).

[142] Y. Yamada, T. Iwase, K. Fujishiro, Y. Uesu, Y. Yamashita, I. Tomeno, and S. Shimanuki, Ferroelectrics 240, 363 (2000).

[143] A. Bussmann-Holder, H. Beige, and G. Völkel, Phys. Rev. B 79, 184111 (2009).

[144] A.S. Shumovsky and V.I. Yukalov, Dokl. Phys. 27, 709 (1982).

[145] J.C. Phillips. Physics of High-T $T_{c}$ Superconductors (Academic, Boston, 1989).

[146] V.I. Yukalov, Int. J. Mod. Phys. B 6, 91 (1992).

[147] A.J. Coleman, E.P. Yukalova, and V.I. Yukalov, Physica C 243, 76 (1995).

[148] V.I. Yukalov and E.P. Yukalova, Phys. Rev. B 70, 224516 (2004).

[149] B.V. Fine and T. Egami, Phys. Rev. B 77, 014519 (2008).

[150] E. Dagotto, T. Hotta, and A. Moreo, Phys. Rep. 344, 1 (2001).

[151] M.B. Salamon and M. Jaime, Rev. Mod. Phys. 73, 583 (2001).

[152] L. Ghivelder and F. Parisi, Phys. Rev. B 71, 184425 (2005).

[153] A.S. Shumovsky and V.I. Yukalov, in International School on High Energy Physics, edited by N.N. Bogolubov (JINR, Dubna, 1983), p. 223.

[154] A.S. Shumovsky and V.I. Yukalov, Phys. Part. Nucl. 16, 569 (1985).

[155] Y.L. Khait, Atomic Diffusion in Solids (Scitec, Zürich, 1997).

[156] V.I. Yukalov, Int. J. Mod. Phys. B 17, 2333 (2003).

[157] V.I. Yukalov, Symmetry 2, 40 (2010). 
[158] A.S. Shumovsky and V.I. Yukalov, Phase States and Transitions (JINR, Dubna, 1985).

[159] C. Rottman and M. Wortis, Phys. Rep. 103, 59 (1984).

[160] M. Volmer, Kinetics of Phase Transitions (Steinkopf, Dresden, 1939).

[161] V.V. Slezov, Phys. Rep. 288, 389 (1998).

[162] V.I. Yukalov, Theor. Math. Phys. 26, 274 (1976).

[163] V.I. Yukalov, Theor. Math. Phys. 28, 652 (1976).

[164] V.I. Yukalov, Phys. Lett. A 81, 249 (1981).

[165] V.I. Yukalov, Phys. Lett. A 81, 433 (1981).

[166] V.I. Yukalov, Phys. Lett. A 85, 68 (1981).

[167] V.I. Yukalov, Physica A 108, 402 (1981).

[168] V.I. Yukalov, Physica A 110, 247 (1982).

[169] V.I. Yukalov, Phys. Rev. B 32, 436 (1985).

[170] V.I. Yukalov, Phys. Lett. A 125, 95 (1987).

[171] V.I. Yukalov, Physica A 141, 352 (1987).

[172] D.B. Fuks and V.A Rokhlin, Beginner's Course in Topology (Springer, Berlin, 2004).

[173] P.C. Hemmer and J.L. Lebowitz, Phase Trans. Crit. Phenom. B 5, 108 (1976).

[174] G.L. Sewell, Phys. Rep. 57, 307 (1980).

[175] N.N. Bogolubov, A.S. Shumovsky, and V.I. Yukalov, Theor. Math. Phys. 60, 921 (1984).

[176] V.I. Yukalov, Laser Phys. Lett. 2, 156 (2005).

[177] V.I. Yukalov, Phys. Lett. A 340, 369 (2005).

[178] V.I. Yukalov, Laser Phys. Lett. 6, 688 (2009).

[179] V.I. Yukalov, J. Phys. Stud. 11, 55 (2007).

[180] S. Floerchinger and C. Wetterich, Phys. Rev. A 79, 063602 (2009).

[181] A.Z. Patashinsky and V.L. Pokrovsky, Fluctuation Theory of Phase Transitions (Pergamon, Oxford, 1979).

[182] G.M. Zaslavsky, Phys. Rep. 80, 157 (1981).

[183] G.M. Zaslavsky, Chaos in Dynamic Systems (Harwood, New York, 1985).

[184] P.T. Landsberg, Physica A 305, 32 (2002).

[185] H. Kleinert, Phys. Rev. Lett 84, 286 (2000). 
[186] V.I. Yukalov, E.P. Yukalova, and V.S. Bagnato, Phys. Rev. A 56, 4845 (1997).

[187] V.I. Yukalov, E.P. Yukalova, and V.S. Bagnato, Laser Phys. 10, 26 (2000).

[188] E.A. Ostrovskaya, Y.S. Kivshar, M. Lisak, B. Hall, F. Cattani, and D. Anderson, Phys. Rev. A 61, 031601 (2000).

[189] D.L. Feder, M.S. Pindzola, L.A. Collins, B.I. Schneider, and C.W. Clark, Phys. Rev. A 62, 053606 (2000).

[190] V.I. Yukalov, E.P. Yukalova, and V.S. Bagnato, Laser Phys. 11, 455 (2001).

[191] Y.S. Kivshar, T.J. Alexander, and S.K. Turitsyn, Phys. Lett. A 278, 225 (2001).

[192] R. Hasson and D. Richards, J. Phys. B 34, 1805 (2001).

[193] R. D’Agosta, B.A. Malomed, and C. Presilla, Laser Phys. 12, 37 (2002).

[194] V.I. Yukalov, E.P. Yukalova, and V.S. Bagnato, Laser Phys. 12, 231 (2002).

[195] V.I. Yukalov, E.P. Yukalova, and V.S. Bagnato, Laser Phys. 12, 1325 (2002).

[196] B. Damski, Z.P. Karkuszewski, K. Sacha, and J. Zakrzewski, Phys. Rev. A 65, 013604 (2002).

[197] R. D’Agosta and C. Presilla, Phys. Rev. A 65, 043609 (2002).

[198] V.I. Yukalov, E.P. Yukalova, and V.S. Bagnato, Phys. Rev. A 66, 043602 (2002).

[199] V.I. Yukalov and E.P. Yukalova, J. Phys. A 35, 8603 (2002).

[200] N.P. Proukakis and P. Lambropoulos, Eur. Phys. J. D 19, 355 (2002).

[201] V.I. Yukalov, E.P. Yukalova, and V.S. Bagnato, Laser Phys. 13, 551 (2003).

[202] V.I. Yukalov, E.P. Yukalova, and V.S. Bagnato, Laser Phys. 13, 861 (2003).

[203] S.K. Adhikari, Phys. Lett. A 308, 302 (2003).

[204] S.K. Adhikari, J. Phys. B 36, 1109 (2003).

[205] P. Muruganandam and S.K. Adhikari, J. Phys. B 36, 2501 (2003).

[206] V.I. Yukalov, K.P. Marzlin, and E.P. Yukalova, Laser Phys. 14, 565 (2004).

[207] V.I. Yukalov, K.P. Marzlin, and E.P. Yukalova, Phys. Rev. A 69, 023620 (2004).

[208] S.K. Adhikari, Phys. Rev. A 69, 063613 (2004).

[209] V.S. Filho, L. Tomio, A. Gammal, and T. Frederico, Phys. Lett. A 325, 420 (2004).

[210] V.I. Yukalov and E.P. Yukalova, J. Low Temp. Phys. 138, 657 (2005).

[211] E.R. Ramos, L. Sanz, V.I. Yukalov, and V.S. Bagnato, Phys. Lett. A 365, 126 (2007). 
[212] E.R. Ramos, L. Sanz, V.I. Yukalov, and V.S. Bagnato, Phys. Rev. A 76, 033608 (2007).

[213] E.R. Ramos, L. Sanz, V.I. Yukalov, and V.S. Bagnato, Nucl. Phys. A 790, 776 (2007).

[214] E.R. Ramos, E.A. Henn, J.A. Seman, M.A. Caracanhas, K.M. Magalhaes, K. Helmerson, V.I. Yukalov, and V.S. Bagnato, Phys. Rev. A 78, 063412 (2008).

[215] V.I. Yukalov and V.S. Bagnato, Laser Phys. Lett. 6, 399 (2009).

[216] J. Williams, R. Walser, J. Cooper, E.A. Cornell, and M. Holland, Phys. Rev. A 61, 033612 (2000).

[217] N.N. Bogolubov and Y.A. Mitropolsky Asymptotic Methods in the Theory of Nonlinear Oscillations (Gordon and Breach, New York, 1961).

[218] G.O. Giacalia, Perturbation Methods in Nonlinear Systems (Springer, New York, 1972).

[219] V.I. Yukalov, Laser Phys. 3, 870 (1993).

[220] N.N. Bogolubov, Problems of Dynamical Theory in Statistical Physics (North-Holland, Amsterdam, 1962).

[221] I. Progogine, Nonequilibrium Statistical Mechanics (Interscience, New York, 1962).

[222] V.I. Yukalov, Phys. Rev. A 79, 052117 (2009).

[223] E. Timmermans, P. Tommasini, M. Hussein, and A. Kerman, Phys. Rep. 315, 199 (1999).

[224] V.I. Yukalov and E.P. Yukalova, Laser Phys. 16, 354 (2006).

[225] V.I. Yukalov and E.P. Yukalova, Phys. Rev. A 73, 022335 (2006).

[226] V.I. Yukalov and E.P. Yukalova, J. Phys. Conf. Ser. 104, 012003 (2008).

[227] V.I. Yukalov, Phys. Rev. Lett. 90, 167905 (2003).

[228] V.I. Yukalov, Phys. Rev. A 68, 022109 (2003).

[229] V.I. Yukalov, Mod. Phys. Lett. B 17, 95 (2003).

[230] S.E. Pollack, D. Dries, M. Junker, Y.P. Chen, T.A. Corcovilos, and R. Hulet, Phys. Rev. Lett. 102, 090402 (2009).

[231] N.R. Cooper, Adv. Phys. 57, 539 (2008).

[232] A.L. Fetter, Rev. Mod. Phys. 81, 647 (2009).

[233] W.F. Vinen and J.J. Niemela, J. Low Temp. Phys. 128, 167 (2002).

[234] W.F. Vinen, J. Low Temp. Phys. 145, 7 (2006).

[235] M. Tsubota, J. Phys. Soc. Jap. 77, 111006 (2008).

[236] M. Tsubota, K. Kasamatsu, and M. Kobayashi, arXiv:1004.5458 (2010). 
[237] V.I. Yukalov, Laser Phys. Lett. 7, 467 (2010).

[238] S.K. Nemirovskii, Phys. Rev. B 77, 214509 (2008).

[239] C. Scheppach, J. Berges, and T. Gasenzer, Phys. Rev. A 81, 033611 (2110).

[240] S.K. Nemirovskii, Phys. Rev. B 81, 064512 (2010).

[241] E.A. Henn, J.A. Seman, G. Roati, K.M. Magalhães, and V.S. Bagnato, Phys. Rev. Lett. 103, 045301 (2009).

[242] V.E. Zakharov, S.L. Musher, and A.M. Rubenchik, Phys. Rep. 129, 285 (1985).

[243] H. Xiong and B. Wu, arXiv:1007.2771 (2010).

[244] M. Rigol, V. Dunjko, and M. Olshanii, Nature 452, 854 (2008).

[245] T. Kinoshita, T. Wenger, and D.S. Weiss, Nature 440, 900 (2006).

[246] E.H. Lieb and W. Liniger, Phys. Rev. 130, 1605 (1963).

[247] E.H. Lieb, Phys. Rev. 130, 1616 (1963).

[248] I.E. Mazets and J. Schmiedmayer, Phys. Rev. A 79, 061603 (2009).

[249] I.E. Mazets and J. Schmiedmayer, arXiv:0912.4493 (2009).

[250] J.A. Seman, E.A. Henn, R.F. Shiozaki, G. Roati, F.J. Poveda-Cuevas, K.M. Magalhães, V.I. Yukalov, M. Tsubota, M. Kobayashi, K. Kasamatsu, and V.S. Bagnato, arXiv:1007.4953 (2010). 


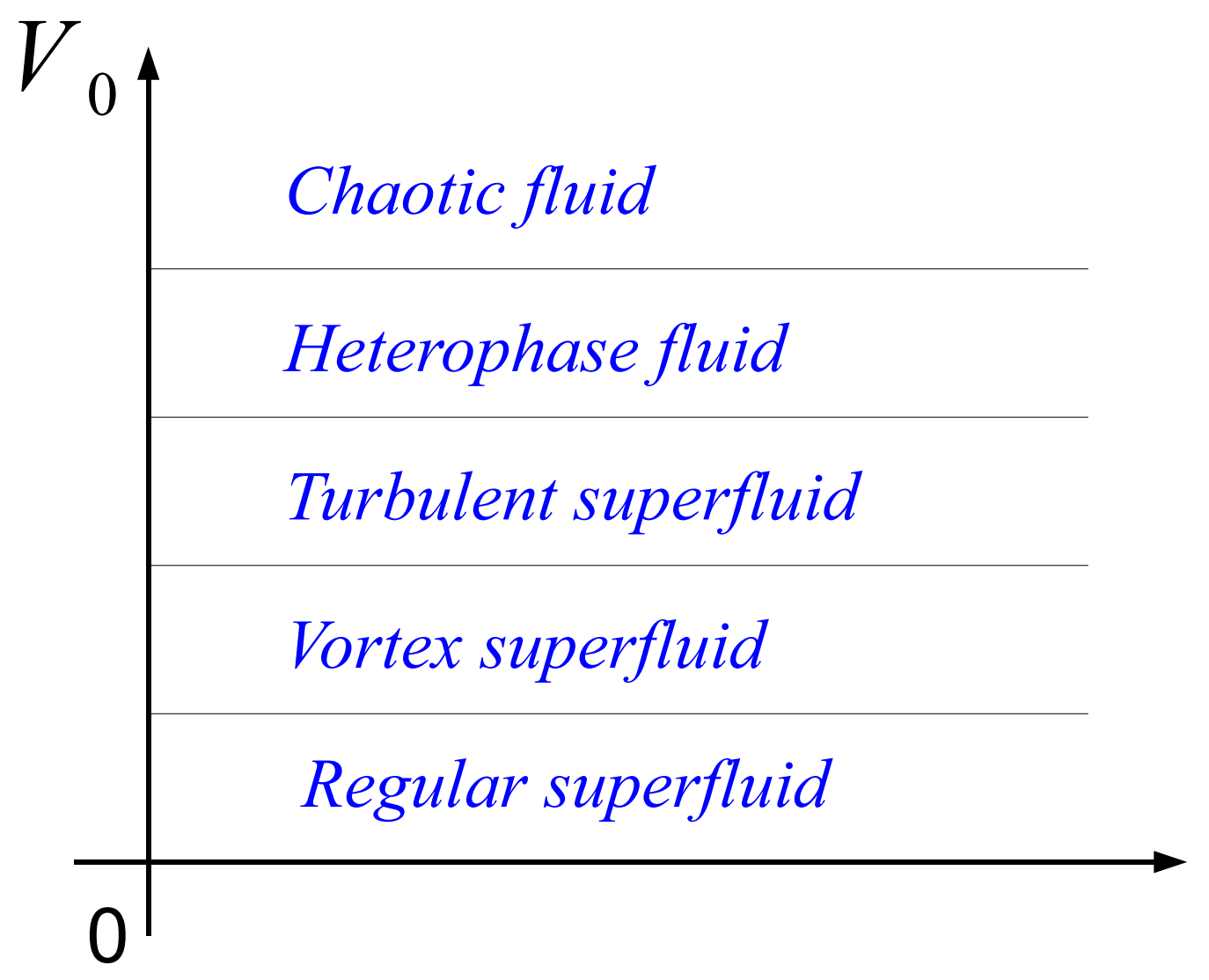

Figure 1: Scheme of the sequence of states for a trapped Bose-condensed system subject to the action of an alternating external field, with the increasing pumped energy $V_{0}$. 